University of Windsor

Scholarship at UWindsor

6-18-2021

\title{
Design of a Regulated Micromachined Air-Sniffer Using Thermal Transpiration Effect for E-Nose Applications
}

\author{
Mukesh Arvind Raju \\ University of Windsor
}

Follow this and additional works at: https://scholar.uwindsor.ca/etd

\section{Recommended Citation}

Raju, Mukesh Arvind, "Design of a Regulated Micromachined Air-Sniffer Using Thermal Transpiration Effect for E-Nose Applications" (2021). Electronic Theses and Dissertations. 8612.

https://scholar.uwindsor.ca/etd/8612

This online database contains the full-text of PhD dissertations and Masters' theses of University of Windsor students from 1954 forward. These documents are made available for personal study and research purposes only, in accordance with the Canadian Copyright Act and the Creative Commons license-CC BY-NC-ND (Attribution, Non-Commercial, No Derivative Works). Under this license, works must always be attributed to the copyright holder (original author), cannot be used for any commercial purposes, and may not be altered. Any other use would require the permission of the copyright holder. Students may inquire about withdrawing their dissertation and/or thesis from this database. For additional inquiries, please contact the repository administrator via email (scholarship@uwindsor.ca) or by telephone at 519-253-3000ext. 3208. 


\title{
Design of a Regulated Micromachined Air-Sniffer Using Thermal Transpiration Effect for E-Nose Applications
}

By

\author{
Mukesh Arvind Raju \\ A Thesis \\ Submitted to the Faculty of Graduate Studies \\ through the Department of Electrical and Computer Engineering \\ in Partial Fulfillment of the Requirements for \\ the Degree of Master of Applied Science \\ at the University of Windsor
}

Windsor, Ontario, Canada

2021

(C) 2021 Mukesh Arvind Raju 


\title{
Design of a Regulated Micromachined Air-Sniffer Using Thermal Transpiration Effect for E-Nose Applications
}

by

\section{Mukesh Arvind Raju}

\begin{abstract}
APPROVED BY:
A. Ahmadi

Department of Electrical and Computer Engineering

O.A. Jianu, Co-Advisor

Department of Mechanical, Automotive and Materials Engineering
\end{abstract}

A. Emadi, Co-Advisor

Department of Electrical and Computer Engineering

April 22nd 2021 


\section{DECLARATION OF CO-AUTHORSHIP/PREVIOUS PUBLICATION}

I. Co-Authorship

I hereby declare that this thesis incorporates material that is result of joint research, as follows:

Chapter 3 and 4 of the thesis is authored by Mukesh Arvind Raju under the supervision of Professor Arezoo Emadi and Professor Ofelia A. Jianu. In all cases, the key ideas, primary contributions, experimental designs, data analysis, interpretation, and writing were performed by the author, and the contribution of the co-author was primarily through the feedback on refinement of ideas and editing of the manuscript.

I am aware of the University of Windsor Senate Policy on Authorship and I certify that I have properly acknowledged the contribution of other researchers to my thesis and have obtained written permission from each of the co-author(s) to include the above material(s) in my thesis.

I certify that, with the above qualification, this thesis, and the research to which it refers, is the product of my own work. 


\section{Previous Publication}

This thesis includes one original paper that is in progress for publication in peer reviewed journals, as follows:

\begin{tabular}{|c|c|c|}
\hline Thesis Chapter & Publication title/full citation & Publication status* \\
\hline Chapter $[3,4]$ & $\begin{array}{l}\text { Raju, M., Jianu, O.A. and Emadi, A., } \\
\text { Design of a Regulated Micromachined } \\
\text { Air-Sniffer Using Thermal Transpiration } \\
\text { Effect for e-Nose Applications. } \\
\text { Proceedings of the Canadian Society for } \\
\text { Mechanical Engineering International } \\
\text { Congress } 2021\end{array}$ & Accepted \\
\hline Chapter [3,4] & $\begin{array}{l}\text { Raju, M., Jianu, O.A. and Emadi, A., } \\
\text { Investigation of Inverse Thermal } \\
\text { Transpiration Effect in the Microchannel } \\
\text { for Gas Sensors. Physics of Fluids } \\
\text { Journal }\end{array}$ & Under Preparation \\
\hline
\end{tabular}

I certify that I have obtained a written permission from the copyright owner(s) to include the above published material(s) in my thesis. I certify that the above material describes work completed during my registration as a graduate student at the University of Windsor. 


\section{General}

I declare that, to the best of my knowledge, my thesis does not infringe upon anyone's copyright nor violate any proprietary rights and that any ideas, techniques, quotations, or any other material from the work of other people included in my thesis, published or otherwise, are fully acknowledged in accordance with the standard referencing practices. Furthermore, to the extent that I have included copyrighted material that surpasses the bounds of fair dealing within the meaning of the Canada Copyright Act, I certify that I have obtained a written permission from the copyright owner(s) to include such material(s) in my thesis.

I declare that this is a true copy of my thesis, including any final revisions, as approved by my thesis committee and the Graduate Studies office, and that this thesis has not been submitted for a higher degree to any other University or Institution. 


\begin{abstract}
Microfluidics artificial olfaction systems are used for plant disease diagnosis in the agricultural field. In an electronic nose, the sniffer draws the air towards an array of gas sensors that detect volatile organic compounds corresponding to diseased plants. The currently available electronic noses involve a mechanical pump of moving parts prone to friction losses, limiting large-scale application. In this work, a microchannel that works on thermal transpiration principle to control the airflow inside it is proposed and designed. It has the potential to be employed as a sniffer component for electronic noses, designed using microelectromechanical systems. COMSOL Multiphysics simulation software is used to identify the design parameters of a three-dimensional microchannel and determine the airflow velocity resulting from the applied temperature using the Navier-Stokes and Energy equation. The heat transfer and fluid flow have been modelled for two different channel geometries (i.e., rectangular, and cylindrical) and two materials (i.e., pyrex and silicon). The proposed microchannel geometries are optimised to obtain the Knudsen number in the range of $0.001<\mathrm{Kn}<0.1$ corresponding to a slip flow regime, for a maximum temperature of $70^{\circ} \mathrm{C}$ at the end of the microchannel, connected to the sensor array. The rectangular microchannel design was chosen to simplify the microfabrication step. The heat transfer capacity and airflow velocity studied by varying the microchannel wall thickness showed that they were unaffected by wall thickness. The influence of temperature on the microchannel and the volume of air pumped in by thermal transpiration is evaluated for four different temperatures, namely $40^{\circ} \mathrm{C}, 50^{\circ} \mathrm{C}, 60^{\circ} \mathrm{C}$ and $70^{\circ} \mathrm{C}$. It is observed that the temperature influences the velocity and volume of air pumped inside the microchannel and $70^{\circ} \mathrm{C}$ projected the highest flowrate velocity with maximum air volume. Natural convection method is applied to calculate the cooling down time for the microchannel, which is in the range of $2.5 \mathrm{Wm}^{-2} \mathrm{~K}$ to $25 \mathrm{Wm}^{-2} \mathrm{~K}$ when exposed to external environment. The microchannel cooling rate is inversely proportional to the heat transfer coefficient. In conclusion, the optimal thermal transpiration effect inside a rectangular microchannel occurred at $70^{\circ} \mathrm{C}$, and the material pyrex can serve as a candidate material for the microchannel fabrication.
\end{abstract}




\section{DEDICATION}

To my beloved father... 


\section{ACKNOWLEDGEMENTS}

I would like to convey my sincere gratitude to my supervisor Dr. Arezoo Emadi for accepting me into the masters' program and supporting me during the course of my study and research. I would also like to thank my co-supervisor Dr. Ofelia A. Jianu for her invaluable time, motivation and for her support in my research. Their guidance and insight have helped me to realise the goal of my research.

I am grateful for my thesis committee members Dr. Rajeev Ruparathna and Dr. Arash Ahmadi for their invaluable time to review my thesis and providing their valuable suggestions and feedback. I would also like to thank my e-Mind and IFuELs research teams for their constructive feedback in our weekly meetings.

A special thanks to my mother and brother for their prayers and love who are miles away. I would like to thank my grandfather for believing in me and supporting me. I would like to thank Mubeena for her love and assistance.

I would like to extend my indebtedness to my friends Barani, Yameema, Jenitha, Siddharth, Dhatchna Moorthy and Subash, for their support and encouragement. I would not have accomplished my goal without your support. 
DECLARATION OF CO-AUTHORSHIP/PREVIOUS PUBLICATION ............iii

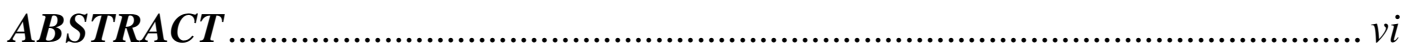

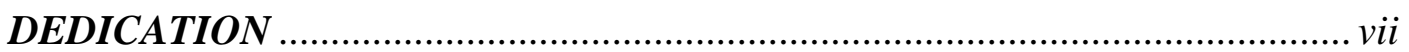

ACKNOWLEDGEMENTS ..................................................................... vii

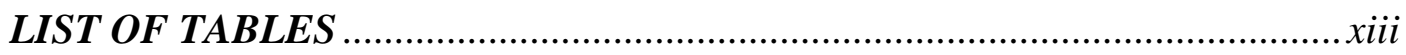

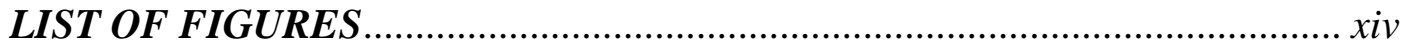

LIST OF ABBREVIATIONS/SYMBOLS ………………....................... xvi

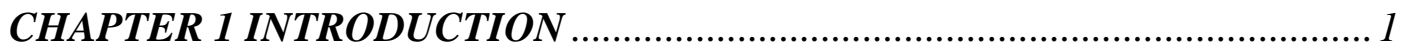

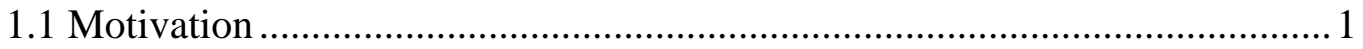

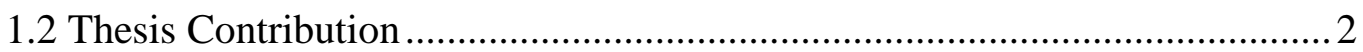

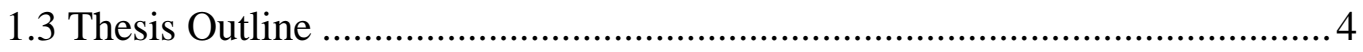

CHAPTER 2 MICROCHANNEL AS E-NOSE SNIFFERS AND GAS FLOW INDUCED IN MICROCHANNEL BY THERMAL TRANSPIRATION EFFECT

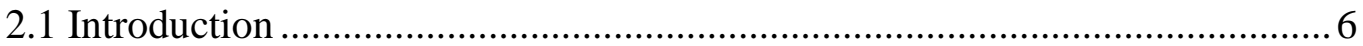

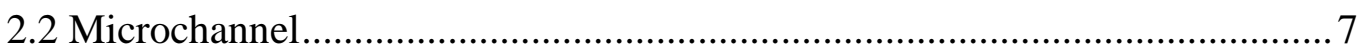

2.3 Classification of Channels Based on Dimensions........................................... 8

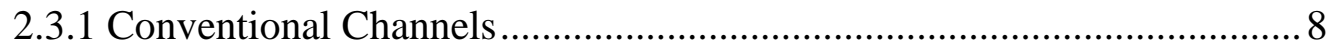

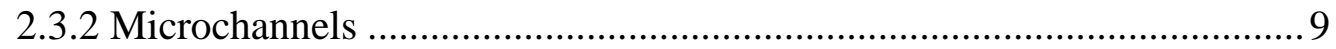

2.4 Types of Microchannels Based on Geometries................................................ 11

2.4.1 Straight Microchannel …………………………................................. 12

2.4.2 Microchannel with Bends .................................................................. 13

2.4.3 Other Microchannel Configurations ........................................................ 13

2.5 Advantages and Disadvantages of Microchannels......................................... 14 
2.6 Microchannel as a Sniffer System............................................................... 15

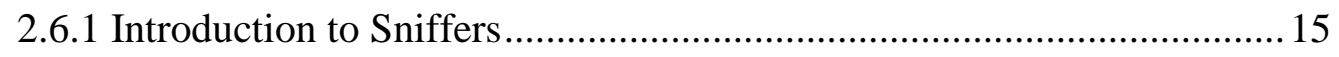

2.6.2 Commercial Sniffers Types ................................................................ 15

2.6.3 Microchannel Sniffers ........................................................................ 16

2.6.4 Advantages and Disadvantages of Sniffers ........................................... 17

2.7 Thermal Transpiration in a Microchannel Sniffer......................................... 18

2.7.1 Introduction to Thermal Transpiration ................................................. 18

2.7.2 Thermal Transpiration Effect in Closed System …………………........ 19

2.7.3 Thermal Transpiration Effect in The Microchannel ...............................2 21

2.8 Types of Flow Induced by Temperature ………………………………........ 23

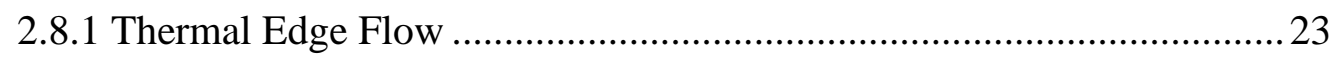

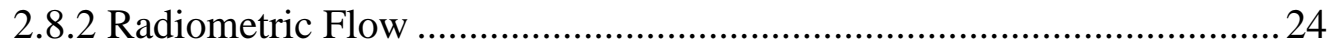

2.8.3 Advantages and Disadvantages of Thermal Induced Flow ……............25

2.9 Analysis of Gas Flow Induced by Temperature in Microchannel .................25

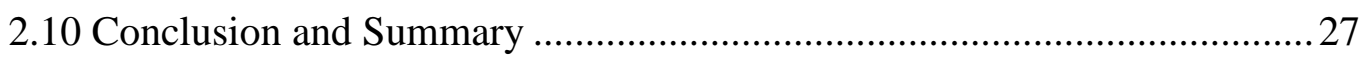

CHAPTER 3 MECHANISM OF OPERATION OF THERMAL TRASNPIRATION EFFECT IN MICROCHANNEL …………….................. 28

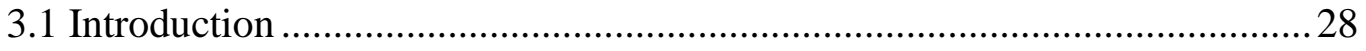

3.2 Heat Source Operating Conditions.............................................................2

3.3 Basic Principle and Mechanism of Operation.................................................2 29

3.3.1 Microchannel Design and Principle ..........................................................29

3.3.2 General Properties of Gas Flow............................................................2 29

3.3.3 Microchannel Design and Material ....................................................... 31

3.3.4 Wall Effects in The Microchannel........................................................... 32

3.3.5 Compressibility Effect in The Fluid ....................................................... 33

3.4 Fluid Flow Analysis Using Navier Stokes Equation.......................................33 
3.5 Temperature Distribution in Microchannel Using Energy Equation ............. 36

3.6 Knudsen Layer Formation in The Microchannel ......................................... 38

3.7 Boundary Conditions to Determine The Fluid Flow ....................................39

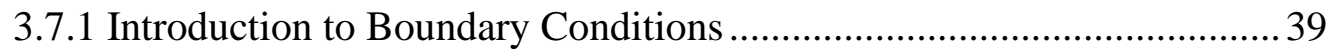

3.7.2 Maxwell Velocity Slip Boundary Condition ..................................... 40

3.7.3 Assumption of Maxwell's Tangential Momentum Accommodation Co-

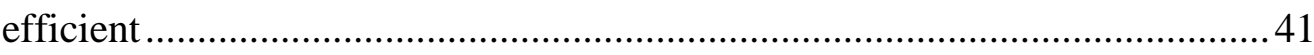

3.7.4 Smoluchowski Temperature Jump Boundary Condition...................... 42

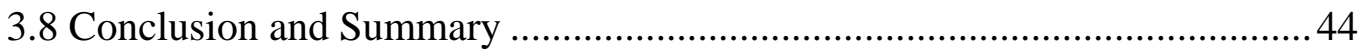

CHAPTER 4 CRITICAL DESIGN PARAMETERS AND AIR FLOW ANALYSIS

IN THE PROPOSED MICROCHANNEL ................................................. 45

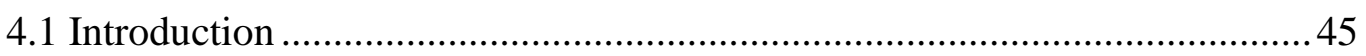

4.2 Finite Element Analysis of Fluid velocity and Heat Transfer in Microchannel

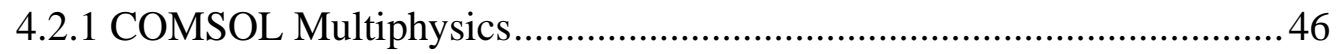

4.2.2 Meshing Characteristics of The Microchannel................................... 47

4.3 Analysis of Boundary Conditions in The Microchannel............................50

4.3.1 Open Boundary in The Microchannel ............................................... 51

4.3.2 Outlet in The Microchannel.......................................................... 51

4.3.3 Temperature Distribution on The Microchannel .................................52

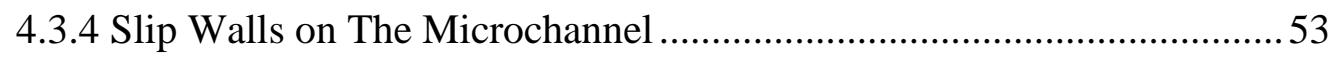

4.4 Reynolds Number in Microchannel ......................................................53

4.5 Investigation of Knudsen Number in The Microchannel ............................55

4.6 Investigation of Velocity in The Microchannel .........................................56

4.7 Investigation of Temperature Distribution in The Microchannel .................58 
4.8 Investigation of Back Flow Velocity of Pyrex Cylindrical and Rectangular

Microchannel.

4.9 Velocity and Temperature Analysis of Pyrex Rectangular Microchannel for

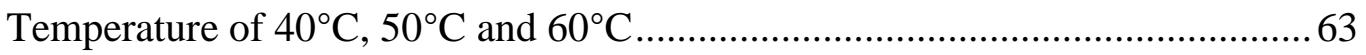

4.10 Volume of Air for Temperature of $40^{\circ} \mathrm{C}, 50^{\circ} \mathrm{C}, 60^{\circ} \mathrm{C}$ and $70^{\circ} \mathrm{C}$...............6 64

4.11 Convective Heat Transfer in Microchannel ............................................6 65

4.11.1 Natural Convection of the Rectangular Pyrex Microchannel...............66

4.12 Investigation of Cool-Down Time Through Natural Convection ...........66

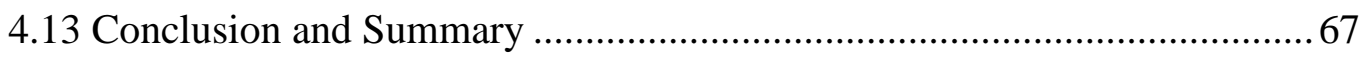

CHAPTER 5 CONCLUSION AND FUTURE WORK .................................. 69

5.1 Conclusion and Summary …............................................................. 70

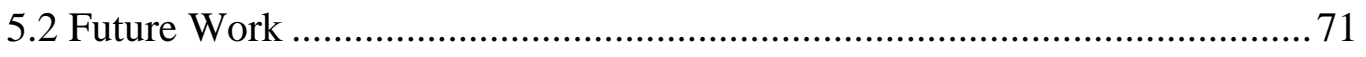

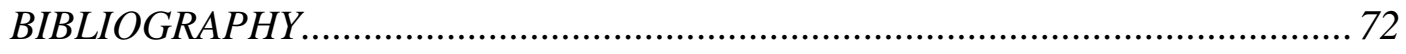

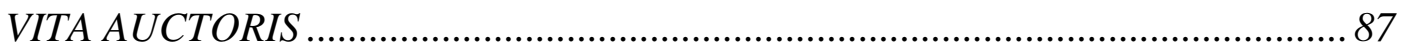




\section{LIST OF TABLES}

Table 2.1 Classification of channels based on hydraulic diameter ......................... 8

Table 2.2 Application of microchannel in various Engineering stream ................. 10

Table 2.3 Classification based on Knudsen number ............................................ 11

Table 2.4 Flow regimes of air through channels of different dimensions ............. 27

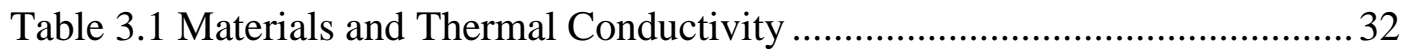

Table 4.1 Dimension of the Cylindrical Microchannel for mesh analysis ............49

Table 4.2 Dimension of the Rectangular Microchannel for mesh analysis ............50

Table 4.3 Classification of Flow based on Reynolds Number ............................54

Table 4.4 Maximum Velocity of air in the cylindrical and rectangular microchannel

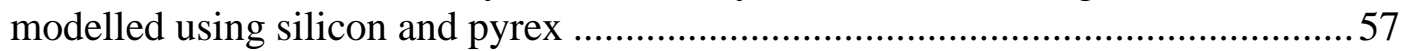

Table 4.5 Time taken to reach $70^{\circ} \mathrm{C}$ from the outlet to inlet for cylindrical and rectangular microchannel modelled using silicon material.

Table 4.6 Time taken to reach $70^{\circ} \mathrm{C}$ from the outlet to inlet for cylindrical and rectangular microchannel modelled using pyrex material. .................................59

Table 4.7 Volume of air sniffed into the microchannel for a temperature of $40^{\circ} \mathrm{C}$,

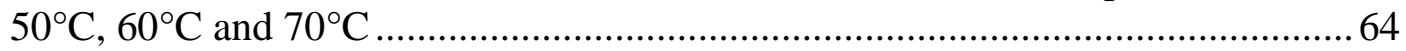

Table 4.8 Cool-down time of the microchannel for different heat transfer co-efficient .66 


\section{LIST OF FIGURES}

Figure 2.1 Schematic diagram of an electronic nose ………................................. 6

Figure 2.2 A straight microchannel with a rectangular cross-section...................... 12

Figure 2.3 A microchannel with T shaped bend ................................................... 13

Figure 2.4 A serpentine-shaped microchannel....................................................... 14

Figure 2.5 A microchannel sniffer showing the gas flow into the inlet................... 16

Figure 2.6 Schematic diagram of the micro gas chromatographer .......................... 17

Figure 2.7 Thermal Transpiration working principle illustration ............................. 19

Figure 2.8 Experimental setup of a circular microtube connected to hot and cold reservoir to compare the Mass flow rate, PVS and TPD for Nitrogen and Helium

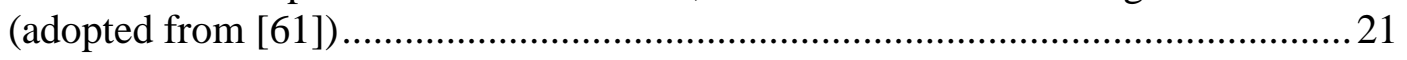

Figure 2.9 Thermal pump in a microchannel..................................................2

Figure 2.10 Classification of thermally induced flow a) Thermal transpiration flow, b) inverted Thermal transpiration flow, c) thermal stress slip flow, d) (nonlinear) thermal stress flow, e) thermal edge flow, f) radiometric flow (adopted from [63][65]). 23

Figure 2.11 Model of Thermal Edge flow (adopted from [69]) ……………….....2. 24

Figure 2.12 Model of Radiometric flow. The sizes of the circular symbols depict the gas velocity, and their colours depict the gas temperature (adopted from [70])....25

Figure 2.13 Flow regimes of gas in a microchannel based on Knudsen number (adopted from [74]). 27

Figure 3.1 Mean free path, diameter, molecular spacing of gas molecules in a chosen

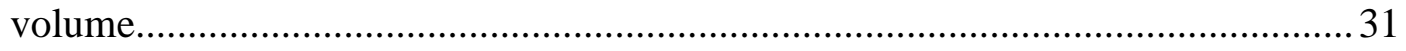

Figure 3.2 The nine components of tensor........................................................... 35

Figure 3.3 Schematic diagram of Knudsen layer......................................................39

Figure 3.4 (a) Perfect (specular) reflection of a fraction of the molecules; (b) Absorption and diffuse reemission of a fraction of the molecules. The circle denotes the gas molecules impinging on the surface ....................................................... 41

Figure 4.1 Main Stages of Finite Element Analysis ................................................46 
Figure 4.2 Finer meshing sequence of (a) cylindrical and (b) rectangular microchannel.

Figure 4.3 Swept meshing sequence of (a) cylindrical and (b) rectangular microchannel.

Figure 4.4 The boundary conditions of the (a) Cylindrical and (b)Rectangular Microchannel

Figure 4.5 3-D Analysis of the temperature distribution in (a) Cylindrical (b) Rectangular microchannel

Figure 4.6 COMSOL simulation to indicate Reynolds number less than 1 for (a) Cylindrical Microchannel (b) Rectangular Microchannel 55

Figure 4.7 Comparing the Knudsen Number for $60 \mu \mathrm{m}$ Cylindrical Microchannel with COMSOL calculation and Analytical calculation 56

Figure 4.8 Comparing the velocity of a cylindrical and rectangular microchannel of hydraulic diameter $60 \mu \mathrm{m}$ with varying thickness of $10 \mu \mathrm{m}, 20 \mu \mathrm{m}$ and $30 \mu \mathrm{m}$ at $70^{\circ} \mathrm{C}$ modelled using (a) silicon-cylindrical, (b) silicon-rectangular, (c) pyrexcylindrical and (d) pyrex-rectangular. 58

Figure 4.9 Comparing the temperature of a cylindrical and rectangular microchannel of hydraulic diameter $60 \mu \mathrm{m}$ with varying thickness of $10 \mu \mathrm{m}, 20 \mu \mathrm{m}$ and $30 \mu \mathrm{m}$ at $70^{\circ} \mathrm{C}$ modelled using (a) silicon-cylindrical, (b) silicon-rectangular, (c) pyrexcylindrical and (d) pyrex-rectangular. 60

Figure 4.10 Air being sniffed into the microchannel at $69.99^{\circ} \mathrm{C} \ldots \ldots \ldots \ldots \ldots \ldots \ldots \ldots \ldots . . . . . . . . . . . . . .61$

Figure 4.11 Air being sniffed out from the microchannel at $70^{\circ} \mathrm{C}$.........................6 62

Figure 4.12 Comparing the backflow velocity of a cylindrical and rectangular microchannel of hydraulic diameter $60 \mu \mathrm{m}$ with varying thickness of $10 \mu \mathrm{m}, 20 \mu \mathrm{m}$ and $30 \mu \mathrm{m}$ at $70^{\circ} \mathrm{C}$ modelled using (a) pyrex-cylindrical and (b) pyrex-rectangular

Figure 4.13 Comparing the (a) Temperature and (b) velocity of a rectangular microchannel of hydraulic diameter $60 \mu \mathrm{m}$ at $70^{\circ} \mathrm{C}$ modelled using pyrex

Figure 4.14 Comparing the volume of air sniffed in a rectangular microchannel of hydraulic diameter $60 \mu \mathrm{m}$ at $40^{\circ} \mathrm{C}, 50^{\circ} \mathrm{C}, 60^{\circ} \mathrm{C}$ and $70^{\circ} \mathrm{C}$ modelled using pyrex.. 65

Figure 4.15 Comparing the cool-down time of a rectangular microchannel of hydraulic diameter $60 \mu \mathrm{m}$ at $70^{\circ} \mathrm{C}$ modelled using pyrex material for the heat transfer coefficient of $\left.2.5 \mathrm{~W} /\left(\mathrm{m}^{2} . \mathrm{K}\right), 10 \mathrm{~W} /\left(\mathrm{m}^{2} . \mathrm{K}\right), 25 \mathrm{~W} /\left(\mathrm{m}^{2} . \mathrm{K}\right)\right)$ 67 


\section{LIST OF ABBREVIATIONS/SYMBOLS}

\begin{tabular}{|c|c|}
\hline Abbreviation & Explanation \\
\hline VOC & Volatile Organic Compounds \\
\hline $\mathrm{D}_{\mathrm{h}}$ & Hydraulic Diameter \\
\hline $\mathrm{Kn}$ & Knudsen Number \\
\hline$\lambda$ & Mean Free Path of Gas Molecules \\
\hline TPD & Thermomolecular Pressure Difference \\
\hline pvs & Pressure-Variation Speed \\
\hline $\mathrm{KP}$ & Knudsen Pump \\
\hline$\delta$ & Molecular Spacing between the Gas Molecules. \\
\hline $\mathrm{n}$ & Number Density \\
\hline $\mathrm{R}$ & Universal Gas Constant \\
\hline $\mathrm{N}_{\mathrm{A}}$ & Avogadro's Number \\
\hline $\mathrm{Ma}$ & Mach Number \\
\hline
\end{tabular}




\section{CHAPTER 1 INTRODUCTION}

\subsection{Motivation}

The Canadian greenhouse vegetable industry is one of the large and rapidly evolving sectors in Canadian horticulture. Ontario continued to lead the greenhouse vegetable sector in 2019, representing 69\% of the total production in Canada, followed by British Columbia and Quebec, with 18\% and 6\% respectively [1]. According to the report from the government of Canada, as of 2019, the nation has imported $\$ 1.6$ billion worth of greenhouse grown vegetables which include tomatoes, peppers, cucumbers, and lettuce. Tomatoes accounted for $37 \%$ of the total greenhouse vegetable sales, followed by cucumbers and peppers, which accounted for $31 \%$ and $28 \%$, respectively [2]. The top two greenhouse vegetable import sources were Mexico, accounting for $88 \%$ and the United States, accounting for 9\% [3].

Pest management is one of the major challenges faced in the greenhouse vegetable industries as the maintenance is very high considering the labour costs involved and the materials that can manage the pest [4]-[6]. Another major problem faced by the greenhouse industry is plant pest diagnosis as pests cannot be identified in their initial stages and becomes visible only after it has caused considerable amount of damage to the plant [7][9]. All plants emit volatile organic compounds (VOCs) which are released from green foliage, reproductive structures such as buds, open flowers, and pollen, and even the roots of plants. The VOCs have high vapour pressure that enables to evaporation in the surrounding air. The VOCs can be sensed by other organisms such as insects [10], [11]. The role of VOCs in order to repel the pesticides and other environmental stressors that can be observed in the early asymptomatic stages. This can be implemented in development of VOC-based crop protection strategies. Early detection can help plan and execute preventive measures against the infection to save the plants.

The traditional methods for analysing the emissions of biogenic VOCs from plants are gas chromatography (GC)-based techniques such as gas chromatography-mass spectrometry (GC-MS), GC with flame ionization detector (GC-FID) [12], [13], and 
thermal desorption-gas chromatography-mass spectrometry (TD-GC-MS) [14] and mass spectrometry based on soft chemical ionization, such as selected ion flow tube-mass spectrometry (SIFT-MS) [15] and proton transfer reaction-mass spectrometry (PTR-MS) [16]. However, rapid non-invasive methods using artificial intelligent noses or electronic noses are emerging as an efficient alternative recently. The artificial electronic noses are coupled with different types of sensor arrays, which transform the VOCs information into an electronic signal. The electronic nose consists of an array of sensors which is integrated with a probe to collect the samples and a Data Application Control (DAC) system which transforms the VOC information into electronic signal [22].

The commercially available sensor systems are bulky and expensive which limits their usage for agricultural applications. There is a need for portable cost-effective Electronic noses (E-nose) based on a small (one-chip) or micro-level sensor array by using integrated circuit (IC) technologies [17]. Most of the devices are connected with an external pumping mechanism. The external mechanical pumping mechanism which comprises of moving parts are prone to malfunctions on constant exposure to environment. The sensitivity, stability and life span of these external pumping mechanism are not good due to the moving parts. In contrast, the non-mechanical micropumps which induces the pumping mechanism by non-mechanical energies such as electric energy, thermal energy, magnetic energy, and chemical energy. These pumps do not have moving parts. A nonmechanical pumping mechanism where the gas flow is induced by thermal gradients in the walls is called Knudsen pump (KP) [18], [19]. The working principle of the KP is based on thermal transpiration effect. The advantages of this pump are that it has no-moving parts, simple structure, low energy consumption, their easy mass production and low energy consumption [20].

\subsection{Thesis Contribution}

This research focuses on investigating the critical design parameter of the cylindrical and rectangular microchannel to analyse the airflow and heat transfer by the applied temperature in the microchannel using the Navier-Stokes and energy equations. The microchannel is used as a sniffer by thermal transpiration effect. 
In this thesis, the microchannel geometries and types are investigated. The channels are classified based on the hydraulic diameter, which categorises the conventional channels and microchannel. The research also focuses on the commercially available sniffers and gas chromatographers which uses external mechanical and non-mechanical pumping mechanism. The gas flow in a microchannel based on the Knudsen number (Kn) is studied. From the baseline study, rectangular and cylindrical microchannels are most commonly used for transporting fluids such as heat sinks and heat exchangers. The rectangular and cylindrical microchannel have simple structure which simplifies the complication in the fabrication process, and they are cost effective. The gas flow based on the flow regimes are studied because the thermal transpiration effect becomes significant at slip flow regime.

The principle of operation to sniff the air through the microchannel is analysed to implement the thermal transpiration effect. The Kn which is used to calculate the degree of rarefaction is calculated using the hydraulic diameter and mean free path. The mean free path which depends on the intermolecular collision of the gas molecules is calculated based on the molecular spacing and molecular diameter of the gas molecules. In the microchannel, the gas flow causes an interaction between the gas molecules and microchannel's walls which results in a formation of layer called Knudsen layer. In order to solve the velocity in the Knudsen layer, Maxwell's and Smoluchowski's boundary conditions are implemented.

The finite element analysis using COMSOL Multiphysics has been implied to calculate the air velocity coupled with the applied temperature in the microchannel. The microchannel is modelled with silicon and pyrex materials to compare the velocity and heat transfer in the microchannel. The dimensions of the microchannel is investigated using finite element analysis by determining the $\mathrm{Kn}$. The wall thickness of the microchannel is varied for both cylindrical and rectangular microchannel modelled using silicon and pyrex to investigate the air velocity and their impact in heat transfer. The volume of air which is following through the rectangular microchannel is analysed for pyrex material for the temperature of $40^{\circ} \mathrm{C}, 50^{\circ} \mathrm{C}, 60^{\circ} \mathrm{C}$ and $70^{\circ} \mathrm{C}$. The back flow velocity is calculated for the cylindrical and rectangular microchannel modelled using pyrex material. 


\subsection{Thesis Outline}

In chapter 2 basic working principles of an electronic nose system and commercially available gas chromatographers are discussed. The external pumping mechanism which is used to collect the samples to the E-nose system comprises of moving parts which are prone to malfunctions. Hence, a thermal pumping mechanism in a microchannel is utilized to collect the sample to the E-nose system. A comprehensive investigation of classification of channel is accomplished. From the literature it is evident that the fluid flow in a conventional channel is not recommended to simulate the thermal transpiration effect because of its diameter. In addition to that various microchannel are investigated based on their geometries. The advantages and disadvantages are analysed to select a candidate geometry that can be used as a sniffer. Finally, the gas flow regimes in a channel are discussed to understand the limits of the characteristic length of the microchannel that has to be modelled in order to fall within the slip flow regime for a maximum temperature of $70^{\circ} \mathrm{C}$.

In chapter 3, the detailed study of the principle of operation of the gas flow in the microchannel by thermal transpiration mechanism is presented. The air velocity is caused by the temperature gradient at the walls of the microchannel. The temperature causes rarefaction effect in air. The air velocity is calculated using he Navier-Stokes equations and the temperature is calculated using the energy equation. The dimensions of the microchannels are in the range of micrometers. This causes the formation of thin layer adjacent to the wall. This layer is called Knudsen layer. The air velocity cannot be calculated using Navier-stokes equations. Thus, boundary conditions are applied to solve the air velocity in the layer.

In chapter 4, the finite element analysis is performed to investigate the cylindrical and rectangular microchannel with silicon and pyrex material. The $\mathrm{Kn}$ is calculated using the mean free path and the analytical values are compared with the COMSOL results in order to investigate the critical design parameters of the cylindrical and rectangular microchannel. The impact in air velocity due to the wall thickness of the microchannel is analysed using Navier-Stokes equations. The influence of wall thickness on the temperature distribution of the microchannel is analysed using the energy equation. The air 
velocity is analysed with temperature of $40^{\circ} \mathrm{C}, 50^{\circ} \mathrm{C}, 60^{\circ} \mathrm{C}$, and $70^{\circ} \mathrm{C}$ to understand the influence of temperature distribution in determining the air velocity. The backflow velocity is calculated for cylindrical and rectangular microchannel modelled using pyrex to compare the air velocity that sniffed into the channel and the air velocity that moves out of the channel. The volume of air which is being sniffed into the microchannel for the temperature of $40^{\circ} \mathrm{C}, 50^{\circ} \mathrm{C}, 60^{\circ} \mathrm{C}$, and $70^{\circ} \mathrm{C}$.

Finally, chapter 5 summarizes the work done in the thesis along with the significant results obtained through this research. Then, potential future works to develop a fabrication procedure and experimental procedure to test the proposed and designed microchannel in this work is discussed. 


\section{CHAPTER 2}

\section{MICROCHANNEL AS E-NOSE SNIFFERS AND GAS FLOW INDUCED IN MICROCHANNEL BY THERMAL TRANSPIRATION EFFECT}

\subsection{Introduction}

Mammals have a complex physiological and anatomical arrangement of specialized cells forming the olfactory system, which functions to sense different odours in the environment. Artificial systems that mimic the olfactory system in mammals have been of interest for decades. These have the potential to detect and recognize individual odours or a mixture of odours in a gaseous sample. These systems are called E-noses [21]. An E-nose system comprises a sniffer, an array of chemical sensors, and systems for signal acquisition and processing, as shown in Figure 2.1. They can contain different arrays of chemical sensors, which are crucial components for detecting diverse gases [22]. The primary mechanism of these E-noses is to produce an electrical change in response to contact with any odour, which can then be compared by a pattern recognition system to identify the odour [23].

A sniffer is a delivery system that transfers the odour from the sample to the sensor array. It is an integral part of an E-nose that exposes the gas sample to the array of sensors and controls the sample volume passing to the sensor. A commonly used aroma delivery system is headspace sampling. This is a procedure that involves the collection of volatile material from a sample. Headspace sampling can be classified into three different types: static headspace, dynamic headspace, and purge methods. In recent advancements of artificial olfaction technology, different kinds of channels are used as sniffers to collect the sample directly from the environment and transport it to the sensors [24].

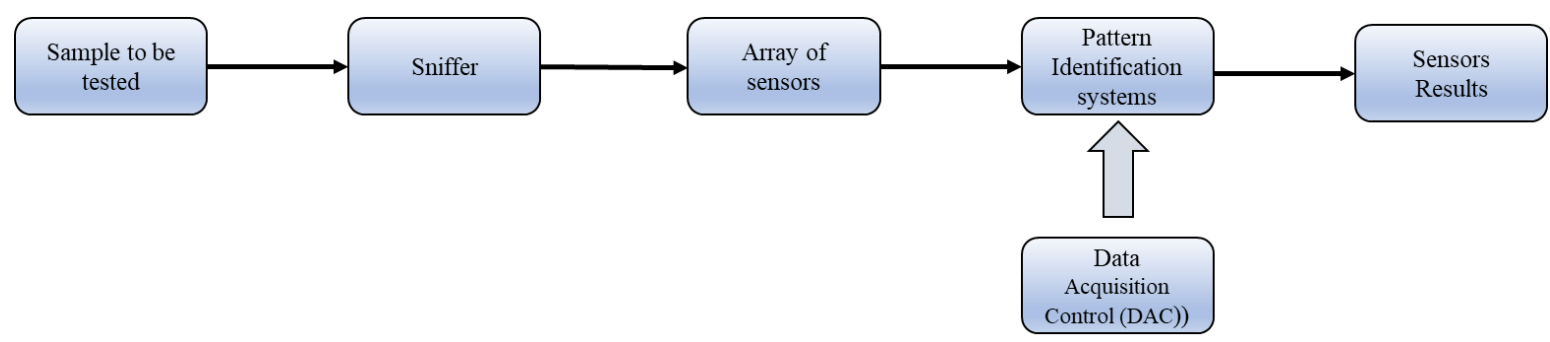

Figure 2.1 Schematic diagram of an electronic nose 


\subsection{Microchannel}

Microchannels are gaining attention in various fields such as microelectronics and biotechnology. These come in circular and non-circular dimensions. Microchannels were initially implemented as heat sinks in electronic devices [25]. The rate of transport of coolant such as air, water depends on the surface area of the channel. The surface area varies with the diameter (D) for a circular channel and Hydraulic diameter $\left(D_{h}\right)$ (refer to section 2.3.2) for a non-circular channel. However, the flow rate of the fluid depends on cross-sectional area which varies linearly with the square of Diameter $\left(\mathrm{D}^{2}\right)$. The surface area to volume ratio of a microchannel varies as 1/D which shows that the diameter decreases, the surface area to volume ratio increases. These are ideal for heat transfer applications as they have high ratio of surface area to volume because of the miniature size. As a result, it makes enhanced convection contrasted to macro-scale or conventional channels. The solid surface ratio is more significant when compared with liquid volume, thus making the heat transfer much higher than conventional channels [26]. It is evident from existing literature [27], [28] that the geometrical dimensions of a microchannel are crucial in deciding its thermal performance. The key feature is the design of the microchannel, which determines its performance such as fluid velocity, heat transfer, wall roughness. There are several designs of microchannels studied previously. However, there is still a need for novel designs for specific applications such as micro-heat exchangers, micro-fluidics, other biomedical applications such as drug delivery system [29].

The application of microchannels is not limited to a heat exchanger for electronic systems, but over the years, they have been introduced in other fields such as automotive, biomedical engineering, refrigerator, and air-conditioning systems. In biomedical engineering, the microchannels are mostly used in drug delivery systems. There is a recent development to incorporate microchannels as a sniffer for E-noses, micro-gas chromatography, and mass spectrometry on a chip to detect different odours from the environment. 


\subsection{Classification of Channels Based on Dimensions}

The introduction of microchannels into various fields has created a need to classify them using standard definitions to provide clarity while referring to them in the scientific literature. There are many classification systems available based on different aspects of the channels. The classification based on the gas flow considerations and hydraulic diameter shown in Table 2.1 was provided by Kandlikar et al. [26].

Table 2.1 Classification of channels based on hydraulic diameter

\begin{tabular}{|l|l|}
\hline Channel Type & Hydraulic diameter $\left(\mathbf{D}_{\mathbf{h}}\right)$ \\
\hline Conventional Channel & $\mathrm{D}_{\mathrm{h}}>33 \mathrm{~mm}$ \\
\hline Mini-Channels & $200 \mu \mathrm{m} \leq \mathrm{D}_{\mathrm{h}} \leq 33 \mathrm{~mm}$ \\
\hline Micro-Channel & $10 \mu \mathrm{m} \leq \mathrm{D}_{\mathrm{h}} \leq 200 \mu \mathrm{m}$ \\
\hline Transitional Channels & $0.1 \mu \mathrm{m} \leq \mathrm{D}_{\mathrm{h}} \leq 10 \mu \mathrm{m}$ \\
\hline Molecular Channels & $\mathrm{D}_{\mathrm{h}} \leq 0.1 \mu \mathrm{m}$ \\
\hline
\end{tabular}

\subsubsection{Conventional Channels}

The conventional channel classification provided by Kandlikar et al. based on the gas flow considerations is essential to identify and apply the channels in electronics appropriately [26]. The hydraulic diameter $\left(\mathrm{D}_{\mathrm{h}}\right)$ of a conventional channel is of the range $3 \mathrm{~mm}$ and above [30]. It also comprises of mini channels under this class which have the hydraulic diameter $\left(D_{h}\right)$ between the range of $200 \mu \mathrm{m}$ to $3 \mathrm{~mm}$. The conventional channel differs from microchannel by the nature of fluid flow and heat transfer. In a conventional channel fluid flow is categorised with no-slip boundary conditions that is the fluid velocity at the channel walls is considered as zero. The velocity of the fluid flow is calculated using Navier-stokes equation with no-slip boundary condition. In a conventional channel the inter-molecular collision is significantly greater than the gas molecules colliding at the wall. When dealing with liquid, the particles close to the surface do not move because adhesive force between solid and liquid is stronger than the cohesive force between the liquids. This imbalance in force causes the velocity at the walls to drop to zero. Hence, the no-slip boundary condition is applied. The nature of the gas flow will be compressible or 
incompressible, laminar, or turbulent, Newtonian, or non-Newtonian. However, in a conventional channel stokes flow will not occur. Stokes flow occurs in microchannel. In a conventional channel the surface area to volume ratio decreases as the diameter of the channel increases. Thus, these channels are not useful to remove heat and can be used to transport gas or liquid in global scale through an external pumping mechanism.

\subsubsection{Microchannels}

The channels with the hydraulic diameter ranging between $10 \mu \mathrm{m}$ to $200 \mu \mathrm{m}$ fall under the microchannel class. This range of the hydraulic diameter is becoming very important for micro-electromechanical system (MEMS) applications. In order to calculate the diameter of a non-circular channel such as rectangular, square, circular, and trapezoidal the term hydraulic diameter is introduced. It is calculated by knowing the area and perimeter of the non-circular channel. It is used to define the nature of gas flow such as laminar, turbulent, creep or stokes flow in a non-circular channel. The hydraulic diameter is used to calculate the dimensionless number such as Reynolds number, Knudsen number [30]. The hydraulic diameter is calculated by four times the channel's flow area divided by the perimeter of the channel [29].

It is given by Equation 2.1.

$$
D_{h}=4 \frac{A}{P}
$$

where $\mathrm{D}_{\mathrm{h}}$ is the hydraulic diameter, $\mathrm{A}$ is the cross-sectional area, and $\mathrm{P}$ is the perimeter of the channel.

Microchannels are among the significant elements in MEMS micro-pumps, microturbines, MEMS pressure sensor, temperature, and velocity measurement [31]. Microchannels are used in various engineering streams which are listed in Table 2.2. 
Table 2.2 Application of microchannel in various engineering stream

\begin{tabular}{|l|l|}
\hline Branches of Engineering & \multicolumn{1}{c|}{ Application } \\
\hline $\begin{array}{l}\text { Electronics and } \\
\text { mechanical }\end{array}$ & $\begin{array}{l}\text { Heat sinks, Heat exchangers [32], } \\
{[33]}\end{array}$ \\
\hline Biomedical & $\begin{array}{l}\text { DNA analysis, blood, protein } \\
\text { synthesis, Biomechanical } \\
\text { applications [34]-[37] }\end{array}$ \\
\hline Chemical & $\begin{array}{l}\text { Electrochemistry, cell trapping, } \\
\text { fluid mixing, lab-on-chip, drug } \\
\text { delivery and drug screening [38]- } \\
\text { [40] }\end{array}$ \\
\hline
\end{tabular}

The gas flow induced in a microchannel by tangential temperature causes rarefaction of the gases. The rarefaction effect causes the gas to move from a colder region to a hotter region under constant initial pressure. The rarefaction effect of gases occurs when the hydraulic diameter is less than $200 \mu \mathrm{m}$. The rarefaction effect occurs when the distance travelled by the gas molecules between two successive collisions is comparable with the hydraulic diameter [41]. Thus, rarefaction effect occurs in microchannels and not in conventional channel as the diameter of conventional channels are significantly higher than the mean free path (refer to section 3.3.2). The tangential temperature causes changes in gas density which causes rarefaction. It is necessary to study the gas flow in microscale because assumption of equilibrium will not be valid to control the flow in a microchannel. The flow pattern in a channel is dependent on the channel size, heat transfer, and pressure drop in the channel. The classification based on $\mathrm{Kn}$ is given in Table 2.3. The mean free path is the distance travelled by the gas molecules between successive collisions in a channel. The Kn, which is the ratio of the mean free path (refer to section 3.3.2) and the hydraulic diameter, influences the rarefaction of gases [42]. 
It is given by Equation 2.2.

$$
K n=\frac{\lambda}{D_{h}}
$$

where $\mathrm{Kn}$ is the Knudsen number, $\lambda$ is the mean free path, and $D_{h}$ is the hydraulic diameter of the mechanical system [42].

Table 2.3 Classification based on Knudsen number

\begin{tabular}{|l|l|l|}
\hline \multicolumn{1}{|c|}{$\begin{array}{c}\text { Range of } \\
\text { Knudsen } \\
\text { Number }\end{array}$} & \multicolumn{1}{c|}{$\begin{array}{c}\text { Type of } \\
\text { Flow }\end{array}$} & \multicolumn{1}{c|}{ Rarefaction Effect } \\
\hline Kn $=0.001$ & $\begin{array}{l}\text { Continuum } \\
\text { flow }\end{array}$ & No Rarefaction effect \\
\hline Kn $=0.1-10$ & Slip Flow & $\begin{array}{l}\text { Rarefaction effect can be modeled with a } \\
\text { modified continuum theory with wall slip } \\
\text { being taken into consideration }\end{array}$ \\
\hline Kn $>10$ & flow & $\begin{array}{l}\text { Between slip flow and free molecular flow, } \\
\text { treated statistically, by the Boltzmann } \\
\text { equation }\end{array}$ \\
\hline molecular & $\begin{array}{l}\text { The motion of individual molecules, that } \\
\text { must be modeled and then treated } \\
\text { statistically. }\end{array}$ \\
\hline
\end{tabular}

\subsection{Types of Microchannels Based on Geometries}

The geometry affects the gas flow in the microchannel. The microchannel geometry are based on the application in different fields of engineering such as mechanical, automotive, and biomedical. Based on the geometries the microchannel are broadly classified as follows, 


\subsubsection{Straight Microchannel}

A straight microchannel can have different geometries such as rectangular, square, circular, trapezoidal, converging and diverging. The geometries describe the crosssectional area of the microchannel. Microchannels that do not consist of any bends in their design are termed as straight microchannels. The straight microchannels are studied in the literature for their simple configuration and application. The gas flow in a straight microchannel has been investigated in studies to understand the flow dynamics of gases and liquids. Numerous experimental and theoretical analysis on microchannel were performed with different assumptions such as slip and no slip boundary condition,

compressible and incompressible flow, different Kn range, different gases and different analytical solutions derived from Navier stokes, Direct Simulation Mote Carlo (DSMC) method, Bhatnagr-Gross-Krook (BGK) method, Brunett and kinetic equation [43]. A representative diagram of a straight microchannel with a rectangular cross-section is shown in Figure 2.2. The traditional microchannel designs predominantly consisted of straight microchannels with variations in the diameter, hydraulic diameter length, width, and height of the channel. These microchannels are used as heat sinks, inlet, and outlet valves in micro-pumps.

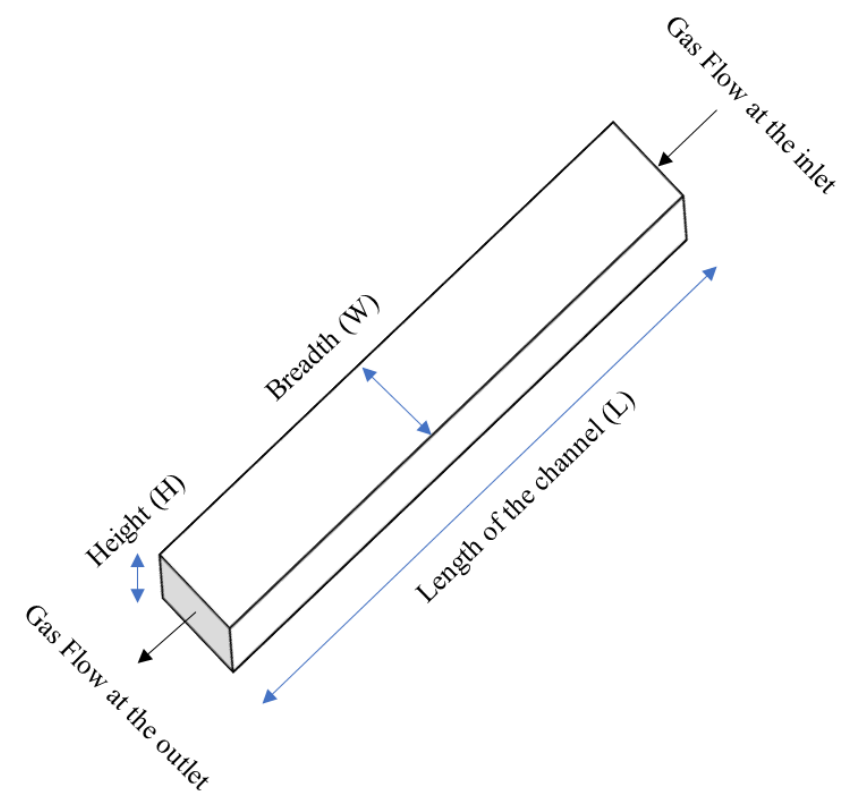

Figure 2.2 A straight microchannel with a rectangular cross-section 


\subsubsection{Microchannel with Bends}

Microchannel became an essential component in the MEMS and microfluidics, facilitating the evolution of complex microchannels with bends. The designs and technological requirements of complicated, intricate systems raised a need for bends and curves in the microchannels. Microchannels with different bends such as $\mathrm{T}$ shaped, Y shaped, and $\mathrm{X}$ shaped are commonly used in microfabricated systems as shown in Figure 2.3. These microchannel has two or more inlets which is used as fluid mixers. These channels are used for analysing the chemical reaction between more than two chemical components. These channels are used as Gas mixer, Lab-on-chip devices [44]-[46]. The gas flow dynamics in the bends, such as the mitre, curved and double turn has been investigated previously. The properties such as gas pressure, mass flow rate, and temperature were monitored in these microchannels. Among the channels, mitre bend was found to have the highest flow rate [47].

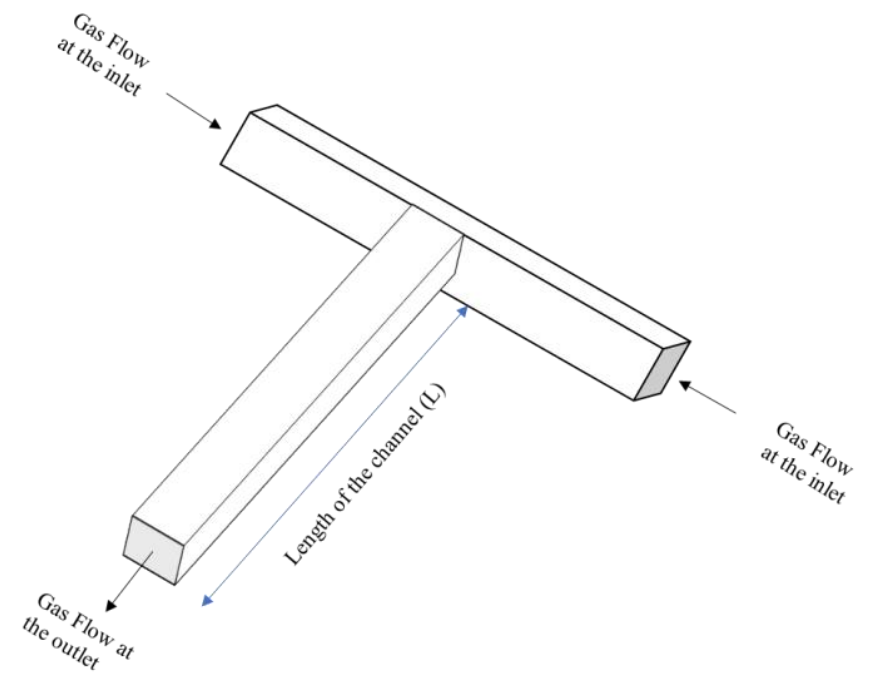

Figure 2.3 A microchannel with T shaped bend

\subsubsection{Other Microchannel Configurations}

There are several non-conventional types of microchannels in various other geometries, such as serpentine, rhombus, sinusoidal, elliptical, isosceles triangular, helical, twisted concentric [48], [49]. A serpentine gas mixer is shown in Figure 2.4. These microchannels are used as gas mixers and lab-on-chip devices. Many experimental and 
theoretical studies were investigated in different geometries of the channel to understand the velocity profile and heat transfer for gases and fluids. These channels are also investigated to be used as heat sink. Analysis on three different channels such as hexagonal, and rhombus are investigated to check the temperature profile, heat transfer coefficient, pressure drop, friction factor, and thermal resistance. For the low hydraulic diameter, the hexagonal channel exhibits highest pressure drop and heat transfer co-efficient when compared with rhombus shaped channel [50]. The fabrication of these microchannel are complex when compared with rectangular or cylindrical microchannel [51], [52]. Most of these microchannels have a pressure-driven flow, and unbalanced pressure causes a possible backflow in them.

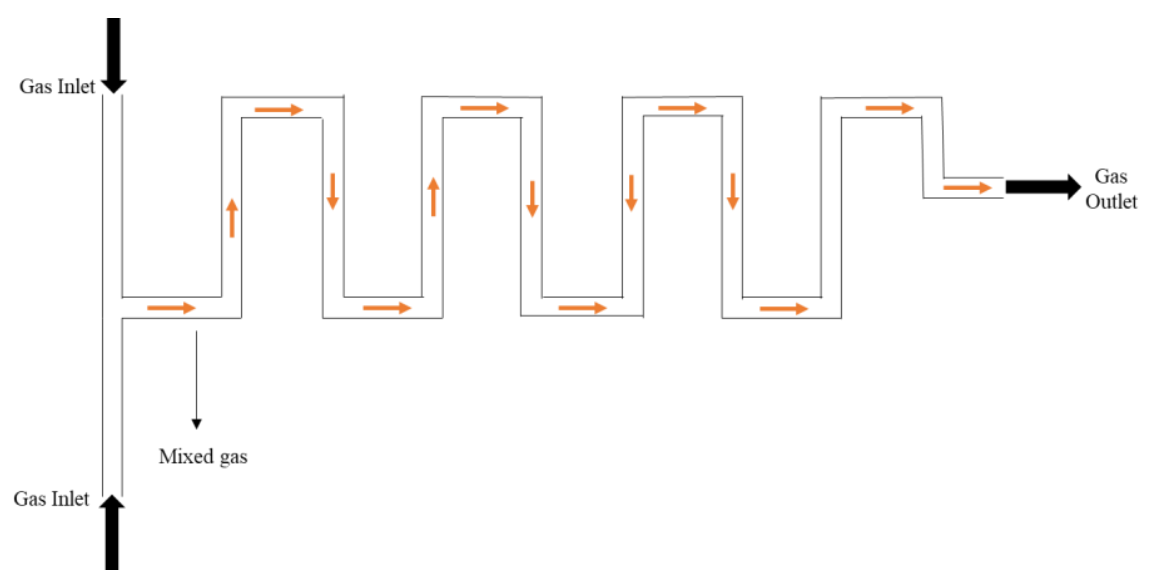

Figure 2.4 A serpentine-shaped microchannel

\subsection{Advantages and Disadvantages of Microchannels}

In conventional channels, the surface area and volume ratio are less when compared with microchannel. Hence, the heat transfer rate of a microchannel is high when compared with conventional channel. The rarefaction effect becomes significant in a microchannel which is a core mechanism of thermal pumping using thermal transpiration effect. The rarefaction effect is not significant in conventional channel. The microchannels are cost effective when compared with conventional channels.

The technological growth in MEMS and microfluidics has made microchannels an integral part of engineering applications. Microchannels have enabled a lower fabrication 
cost and overall performance enhancement for various applications such as heat sinks, heat exchangers, and drug delivery systems. The one disadvantage of microchannels with bends is that they induce a secondary flow at the bend region of the channel, which causes a pressure loss [47]. Hence a straight microchannel is a candidate choice for being used as a sniffer in a MEMS-based electronic nose.

\subsection{Microchannel as a Sniffer System}

E-noses detect the odour by sensing the chemical compounds present in the sample using an array of sensors. E-nose technology is implied in varies industries such as agricultural, environmental, food processing and manufacturing. A sniffer is a component that sniffs the odour from the sample and pass those samples to the sensor array of the Enose system.

\subsubsection{Introduction to Sniffers}

A sniffer is defined as a device that can inhale a specific volume of air in which different VOCs can be detected and differentiated. These utilize pumps to flow the air sample into the sensor compartment, which, based on pattern recognition, identifies the VOCs present. The sample air can be drawn inside by creating a vacuum for aspiration, or it can be injected inside by a syringe or peristaltic pump. The inlet channel that transports the sample to the detector or array of sensors can be macroscopic or microscopic [53]. MEMS-based microchannel sniffers can be an alternative to the existing commercial sniffers available. There are different commercial sniffers in the market currently [54].

\subsubsection{Commercial Sniffers Types}

Most of the commercial sniffers are a large table-top setup of an analytical instrument. The Smith Detection Cyranose is one of such that has an internal pump to draw the sample through a wand-type inlet with a flared shape end that can be considered as a sniffer [54]. The sniffers can be of a single technology or combined technology type. The single technology sniffers work on sensors based on principles such as metal oxide sensors, surface acoustic, and bulk acoustic wave technology, colorimetric sensors, catalytic fieldeffect sensors, and Electrochemical sensors. 
The combined technology sniffers work in combination with a traditional analytical technique such as gas chromatography and mass spectroscopy.

Airsense Analytics [55] has developed PEN2 and PEN3 with intelligent sensors to identify the gases and vapours. It has a flow rate adjustment feature and uses an internal pump mechanism to sample air. There are other single technology sniffers such as FOX2000 from Alpha MOS, Artinose from Sysca, and Cybernose from CSIRO [56]. ZNose 4200 works on a Surface Acoustic Wave (SAW) sensor and gas chromatography technology with an internal pump to draw the sample. There are other combined technology sniffers such as GDA2 from Airsense Analytics, Area Monitor from Rae Systems [24].

\subsubsection{Microchannel Sniffers}

Microchannels are components of MEMS such as micro-pumps, micro-sensors, micro-turbines, and sensors for pressure. The study published by Bernardini et al. [57] described a microdevice consisting of a microchannel, including an integrated gas sensor with a heater. A rectangular microchannel was used to sniff the gas using a Thermal transpiration phenomenon, which is a gas movement from cold chamber to hot chamber caused by a force when a channel connects two chambers of the same pressure and different temperature. In this device, the heater acts as a sensor that is placed in the microchannel [57]. A representative image of a microchannel sniffer is shown in Figure 2.5.

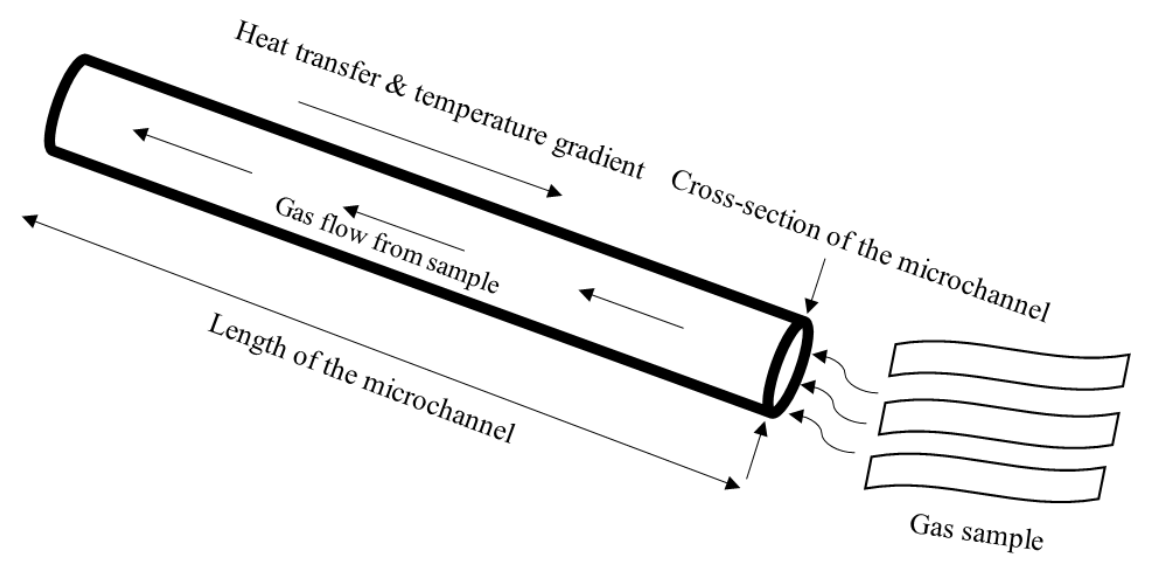

Figure 2.5 A microchannel sniffer showing the gas flow into the inlet 
In a recent study [54], a micro gas chromatographer is developed with a pump and a gas chromatography compartment. In this method, a bidirectional KP intake the air. It avoids the need for an inlet valve. The pump functions by providing negative pressure in the separation module and by providing a positive pressure chromatographer module. The temperature gradient across the channel pumps the air to a preconcentration column before sending it to the chromatography columns. The gas intake using the KP is controlled using a flow meter. The gas flow diagram is shown in Figure 2.6. In another study [58], a chipscale micro gas chromatographer was designed with a T-shaped inlet to draw the gas sample.

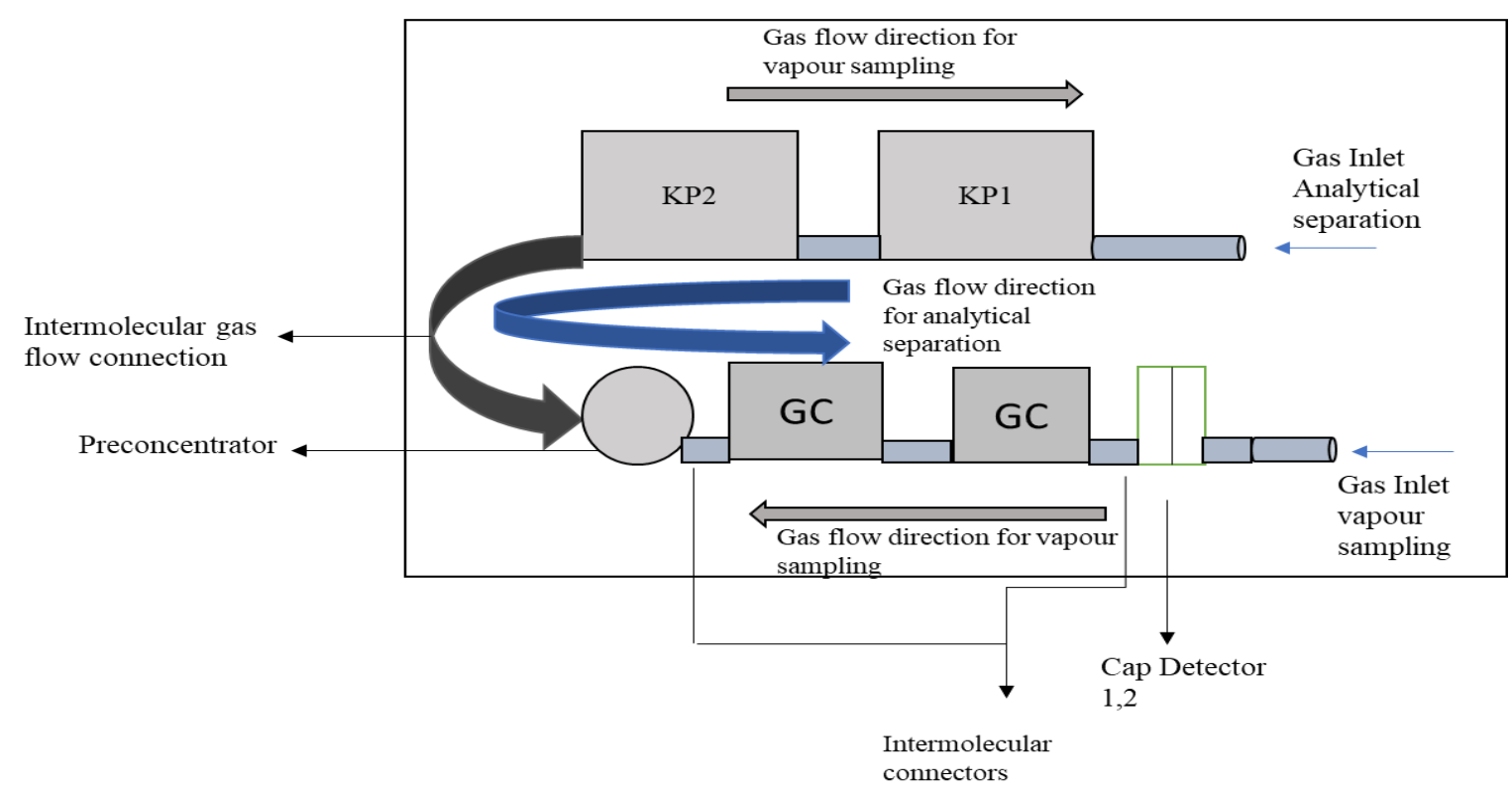

Figure 2.6 Schematic diagram of the micro gas chromatographer

\subsubsection{Advantages and Disadvantages of Sniffers}

The commercial sniffers are usually large and bulky. The analytical techniques are destructive, where they require to house the sample to be detected or diagnosed in a closed container and collect the gas sample by headspace sampling. There is a need for nondestructive methods that can be portable and handheld to be used in the field, such as plant pathology diagnosis, explosives detection, and gas leak in industries. The MEMS-based microchannel sniffers can serve as candidate technology and help reduce the production, 
maintenance, and per-unit cost. They can provide quick real-time detection without reagents and laboratory space for analysis [24].

\subsection{Thermal Transpiration in a Microchannel Sniffer}

The principle of non-mechanical micro pumping mechanism where the gas flow is induced by temperature gradients was proposed by Knudsen. The pump works on the principle called Thermal transpiration effect which is explained in section 2.7.1.

\subsubsection{Introduction to Thermal Transpiration}

Thermal transpiration or Thermal creep is a phenomenon discovered by Reynolds in 1878 [59], according to which when a microchannel connects two chambers without any pressure difference, then the gas from the cold chamber will move towards the hot chamber through the channel [59]. The working principle is illustrated in Figure 2.7. Thermal transpiration has significant effects on the pressure, velocity, and temperature of the gases in a microchannel. The tangential temperature across the solid walls of the microchannel, provokes a force that makes the gas move from colder place to a hotter place. The investigation was carried using plaster-of-Paris plug separated using two reservoirs ( 1 and 2) [59]. The investigation showed that at low densities the equilibrium pressure on the two sides of the microchannel is given using the equation 2.3.

$$
\frac{P 2}{P 1}=\left(\frac{T 2}{T 1}\right)^{1 / 2}
$$

where, $\mathrm{P} 2$ and $\mathrm{P} 1$ are the pressure at the hot and cold sides of the microchannel and T2 and $\mathrm{T} 1$ are the temperature at the hot and cold reservoir. $\frac{P 2}{P 1}$ is called as the thermomolecular pressure difference (TPD). The KP or compressor concept, which works on the principle of thermal transpiration, was discovered by Knudsen [60]. 


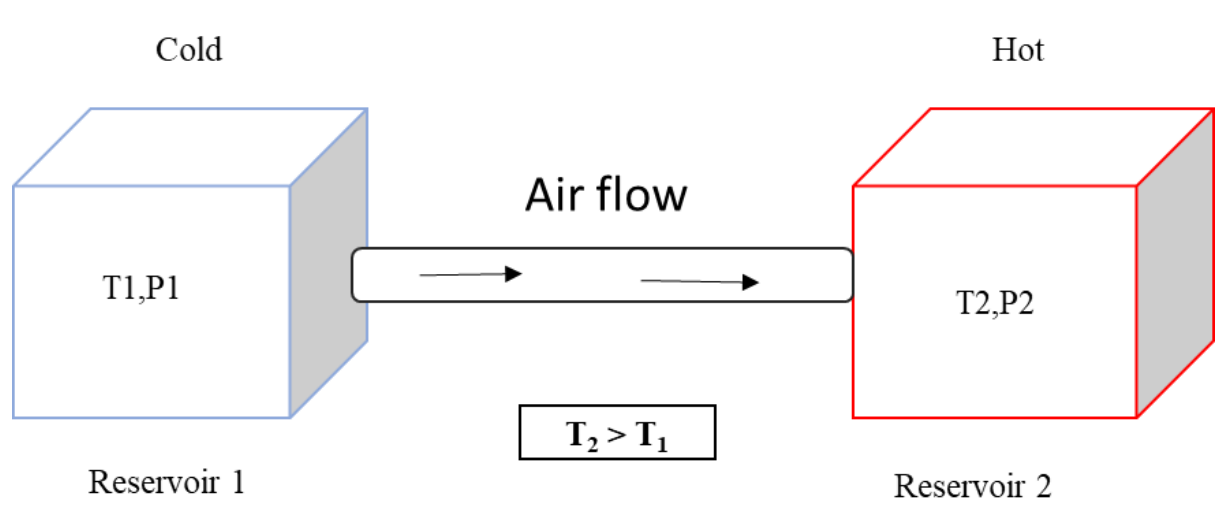

Figure 2.7 Thermal Transpiration working principle illustration

\subsubsection{Thermal Transpiration Effect in Closed System}

The thermal transpiration effect is experimented using nitrogen and helium gas in a closed system by Rojas.et.al [61]. The experiment was conducted using a pyrex microtube as shown in Figure 2.8. The hydraulic diameter (refer to chapter 3.3.2) of the microchannel is $490 \pm 1 \mu \mathrm{m}$ and the length of the microtube is $3.053 \pm 1 \mathrm{~cm}$. Three different temperature difference of $50^{\circ} \mathrm{C}, 60^{\circ} \mathrm{C}$ and $70^{\circ} \mathrm{C}$ are maintained between the hot and cold chambers between the two chambers. The imposed temperature gradients were monitored using the IR camera which showed that the temperature difference is linear on the microtube's surface. The hot and cold reservoir are connected with a high-speed capacitance diaphragm gauge (CDG). The temperature difference is maintained stable during the whole experiment. The internal rings which are connected with the microtube's end are connected with valve A to dampen pressure oscillation produced by the vacuum system. The external opening ring which is connected with two butter-fly valves which is connected with two high pressure tanks containing nitrogen and helium. The experiment comprises of four stages.

$>\quad$ Stage 1: After imposing the gas into the chamber and stabilizing the pressure inside the chamber, valve $\mathrm{B}$ and $\mathrm{C}$ are closed. These two valves will remain closed for the whole experiment. The valve A remains open in order to distribute the pressure throughout the microtube. A stationary macroscopic displacement of gas occurs from cold chamber to the 
hot chamber through the microtube. The temperature gradient creates the gradient of density in the direction of the microtube's axis. The gas motion reduces the density gradient. Thus, the gas flows from higher density to lower density region from cold chamber to hot chamber. The cold chamber is considered as the gas source volume and the hot chamber is considered as the gas storage volume.

Stage 2: This stage is called as the first phase of transitional stage. The pressure varies with time linearly. The pressure variation increases at the hot reservoir while decreasing in the cold reservoir. The pressure gauges monitor the pressure variation with time. The pressure variation is linear.

Stage 3: This stage is called the second phase of the transitional stage. The pressure varies non-linearly with time. The pressure-variation speed (pvs) decreases, and it depends on the continuous increase of pressure difference between the two reservoirs. Thus, the pressure difference creates a counter flow which is a reverse thermal transpiration flow. This flow is called pressure driven flow.

$>\quad$ Stage 4: The counter flow will completely balance the thermal transpiration flow at the final stage of the experiment. The mass flow rate of gas becomes zero when the pressure difference increases. Thus, the system is reaches final equilibrium stage where the mean mass flow rate is zero. At this stage, the TDP is less than 1 at the cold chamber and TDP is greater than 1 at the hot chamber.

Thus, from the experiment, the TDP and pvs depends on the gas physical properties. Due to different molecular mass, helium has a higher pvs and TDP than nitrogen. The temperature difference is a key factor in determining the mass flow rate. Higher temperature difference leads to increased value of the mass flow rate intensity. Helium which is lighter than nitrogen has high mass flow rate. 


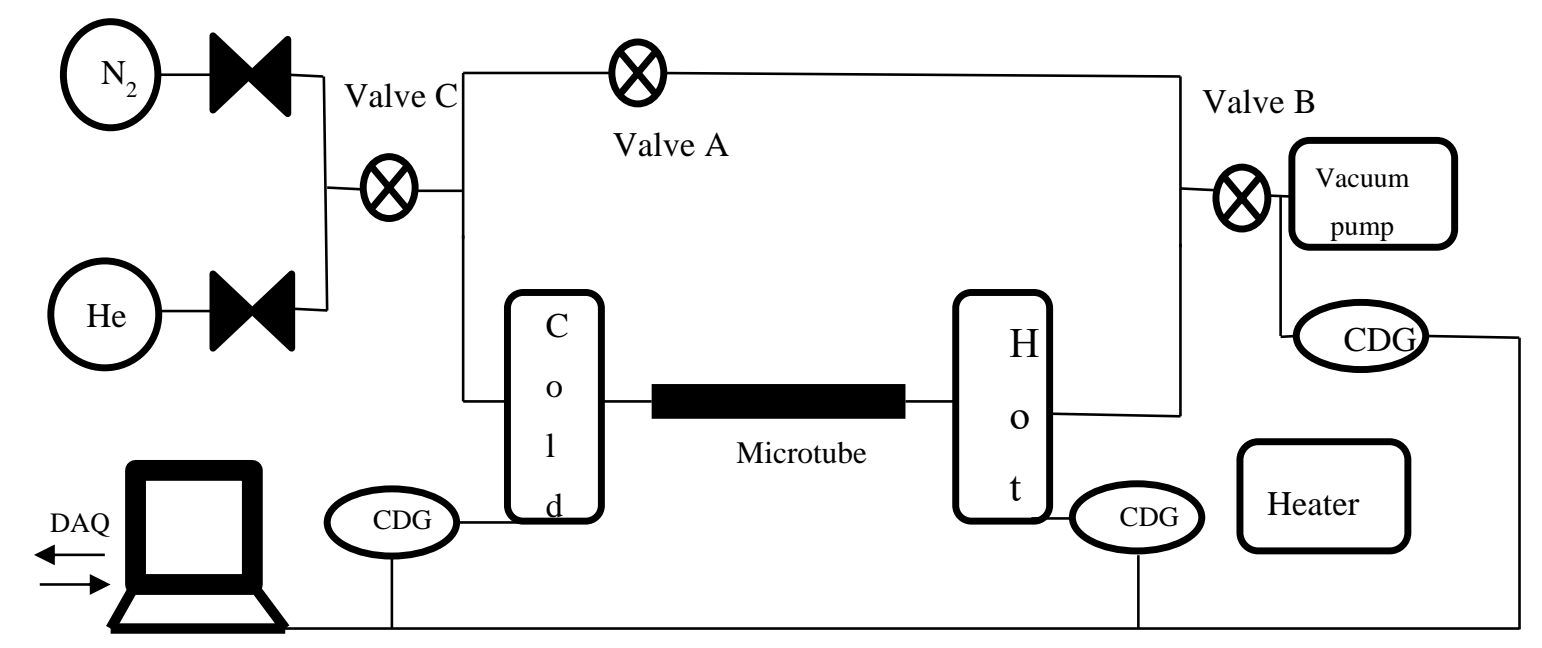

Figure 2.8 Experimental setup of a circular microtube connected to hot and cold reservoir to compare the Mass flow rate, PVS and TPD for Nitrogen and Helium (adopted from [61])

\subsubsection{Thermal Transpiration Effect in The Microchannel}

The basic knowledge of flow and thermal properties at the microscale is essential in understanding the differences between macroscale and applying it to technological needs [62]. The Thermal transpiration in a microchannel caused by temperature gradient generated across the wall that causes movement of the gas, which is shown in Figure 2.9. The microchannel is etched in a silicon substrate and a microheater is placed at the outlet of the microchannel's surface. Thus, the temperature gradient induces a flow from the microchannel's inlet to the microchannel's outlet where the microheater is positioned. According to classical fluid dynamics, there is no time-dependent flow induced by virtue of only temperature field without any external forces such as gravity and pressure. However, when the Kn number is greater than equal to 0.0001 , the temperature gradient influences a flow. Thus, making the temperature field an important parameter to induce a time-dependent flow. This gas flow caused by temperature can be classified as the following types: Thermal transpiration flow, inverted Thermal transpiration flow, thermal stress slip flow, thermal edge flow, and radiometric flow, as shown in Figure 2.10 [63][65].

The recent advancement in MEMS microchannel sniffers has brought attention to various kinds of micropumps that work based on different actuating principles. These 
pumps can be mechanical or non-mechanical, and the thermal transpiration based nonmechanical pump. The non-mechanical pumps do not contain any moving parts. Microchannels are crucial in the KP based on thermal transpiration [62]. The microchannel dimensions influence the gas to be in a rarefied state and determine the performance of the KPs. There are KPs already in use for flow delivery in micro gas chromatography. The application of KPs can be classified as vacuum generation, flow delivery, and gas separation [66]-[68].

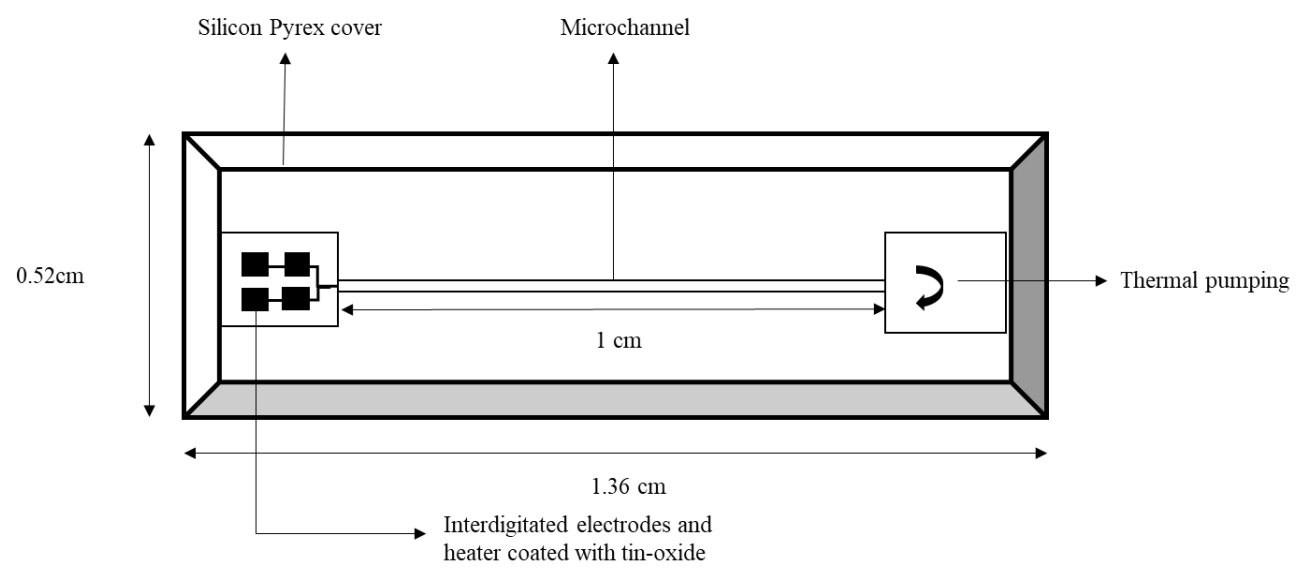

Figure 2.9 Thermal pump in a microchannel 
a)

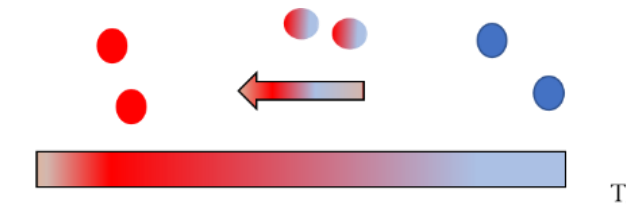

c)

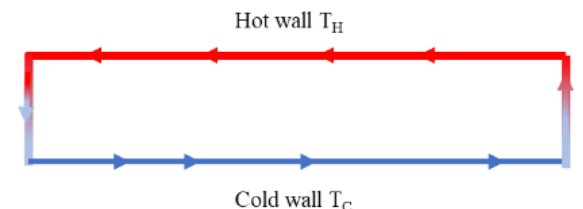

e)

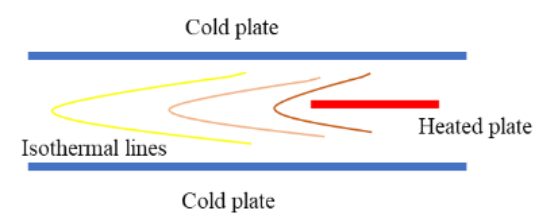

b)

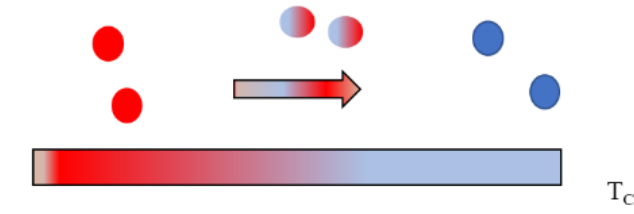

d)

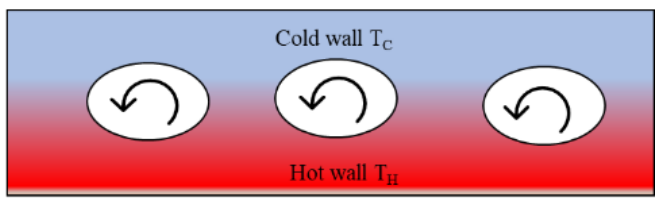

f)

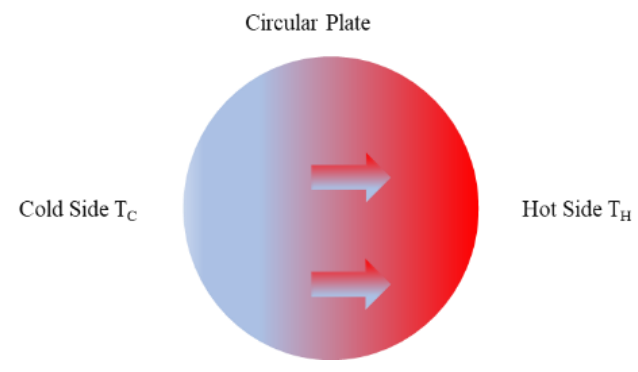

Figure 2.10 Classification of thermally induced flow a) Thermal transpiration flow, $b$ ) inverted Thermal transpiration flow, c) thermal stress slip flow, d) (nonlinear) thermal stress flow, e) thermal edge flow, $f$ ) radiometric flow (adopted from [63]-[65])

\subsection{Types of Flow Induced by Temperature}

Thermal flows are induced by temperature gradient. The thermal flows becomes significant in micro-scale devices such as microchannel and two parallel plates separated by micrometer distance. As shown in Figure 2.10, the thermally induced flow becomes significant when the Kn ranges from 0.001 to 0.1 . The types of thermally induced flows is as follows,

\subsubsection{Thermal Edge Flow}

The thermal transpiration effect, which is discussed in section 2.7.1, the microchannel's boundaries are assumed to be smooth without any edges in the inner walls of the microchannel. The microchannel boundaries are assumed to have a non-isothermal surface to induce a flow. In thermal edge flow and in radiometric flow an isothermal surface 
triggers the flow. The thermal edge flow was discovered by Aoki et al. [64] and experimentally verified by Sone and Yoshimoto [69]. The thermal edge flow occurs in a channel comprising of parallel heated and unheated plate as shown in Figure 2.11 [69]. The isothermal plates near the edge of the cold plates triggers the flow as shown in Figure 2.11.

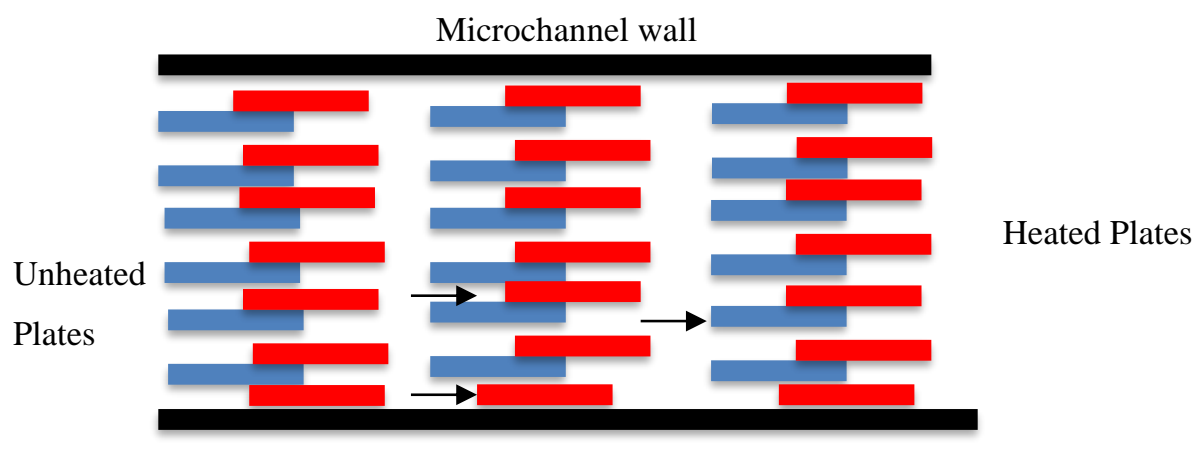

Figure 2.11 Model of Thermal Edge flow (adopted from [69])

\subsubsection{Radiometric Flow}

The radiometric flow is obtained by changing the accommodation coefficient of the adjacent walls. It is obtained by adjusting the temperature of the ratchet walls as shown in Figure 2.12 [70]. It is a thermalizing process of gas molecules that colliding at the walls. The gas molecules possess weak or zero thermalization when colliding at the cold side of the ratchet walls which is the specular surface. On the contrary the gas molecules collide with the hot surface which enhances the thermalization of the gas molecules. As a result, gas flow occurs from cold side to hot side. The radiometric flow is implied in Crooke's radiometer [71], [72]. 


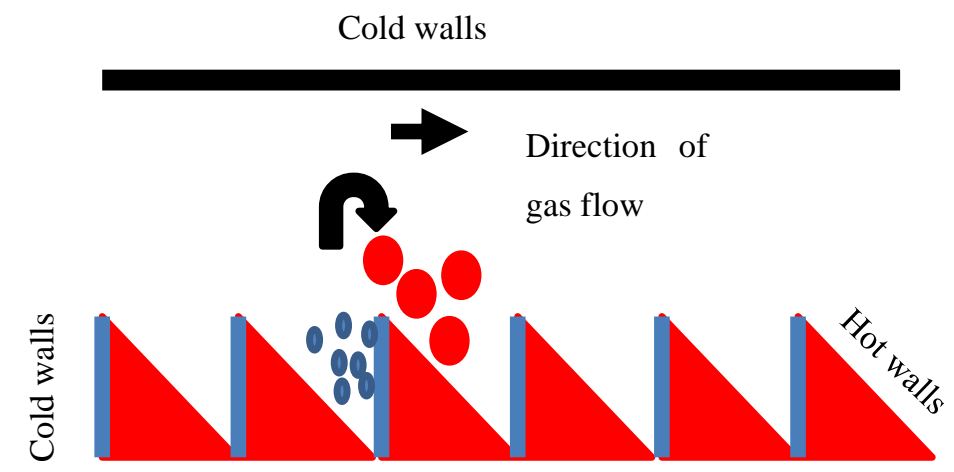

Figure 2.12 Model of Radiometric flow. The sizes of the circular symbols depict the gas velocity, and their colours depict the gas temperature (adopted from [70])

\subsubsection{Advantages and Disadvantages of Thermal Induced Flow}

The thermal pump based microchannel sniffers have the advantage of not consisting of any moving part and hence will last longer. They are simple to design and construct with low energy consumption for gas sample delivery systems, making them sustainable. A straight microchannel appears to be a potential candidate for a thermal pump-based sniffer as it will have low cost, less fabrication complexity and high performance [73]. The Thermal edge flows can be formed on constant temperature walls, eliminating the need for temperature gradients necessary in Thermal transpiration flow. This reduces the requirement of temperature control and therefore makes thermal edge flow easier to apply in practise. However, in terms of fabrication it is complex to fabricate ratchets shaped wall and heat plates which are adjacent to each other in a small dimension of the microchannel. Hence, thermal transpiration flow is chosen as a candidate choice to induce gas flow in a microchannel.

\subsection{Analysis of Gas Flow Induced by Temperature in Microchannel}

The gaseous flow through a microchannel has different properties than conventional channels due to the miniaturized size [62]. Usually, slip-flow regimes are modeled based on Navier-Stokes and energy equations by considering boundary conditions in order to include rarefaction effects on the velocity and temperature fields. The rarefied gas flow in the microchannel is because of its small characteristics dimension and the density change of the gas at the inlet and outlet of the channel. Hence, the gas flow and heat transfer in the 
microchannel should be investigated because of its applications in MEMS devices. The degree of rarefaction depends on $\mathrm{Kn}$ where the flow regime of the gas is classified as shown in Figure 2.13 [74]. The KP are commonly used in MEMS applications which has no moving parts and operates in a slip flow regime with gas flow as their primary functionality.

The flow in a microchannel is determined by the rarefaction of the gases. The increase in rarefaction effects can cause the $\mathrm{Kn}$ to increase, and the continuum assumption collapses [75]. Thus, the increase in the rarefaction of a gas causes a decrease in the mass flow rate. It affects the flow of the gases through the channel. The Navier -stokes equations ideally deals with the nonlinear pressure, temperature, and velocity distribution that are integrated with time used to calculate the gas flow rate with time. The pressure in the channel decreases to overcome the friction at the walls of the channel. Friction at the walls and pressure are inversely proportional. For a constant temperature gradient, the pressure in the channel drops down, and the velocity of the gas increases with an increase in time [76].

In a continuum approach, the flow of the gas or fluid is continuous, whereas, in the molecular approach, the flow varies depending upon the rarefaction of the gas. The rarefaction is caused by the temperature gradient, which causes the non-isothermal flow [77]. The flow rate at the outlet of the channel is always greater when compared with the inlet because of the heat source. The aspect ratio and pressure ratio in a microchannel have an impact on the temperature gradient. The aspect ratio and pressure ratio are inversely proportional to the temperature gradient [77]. The flow rate depends on the temperature gradient at the walls of the channel.

For example, air passing through channels of different dimensions experiences different flow regimes, as shown in Table 2.4. 

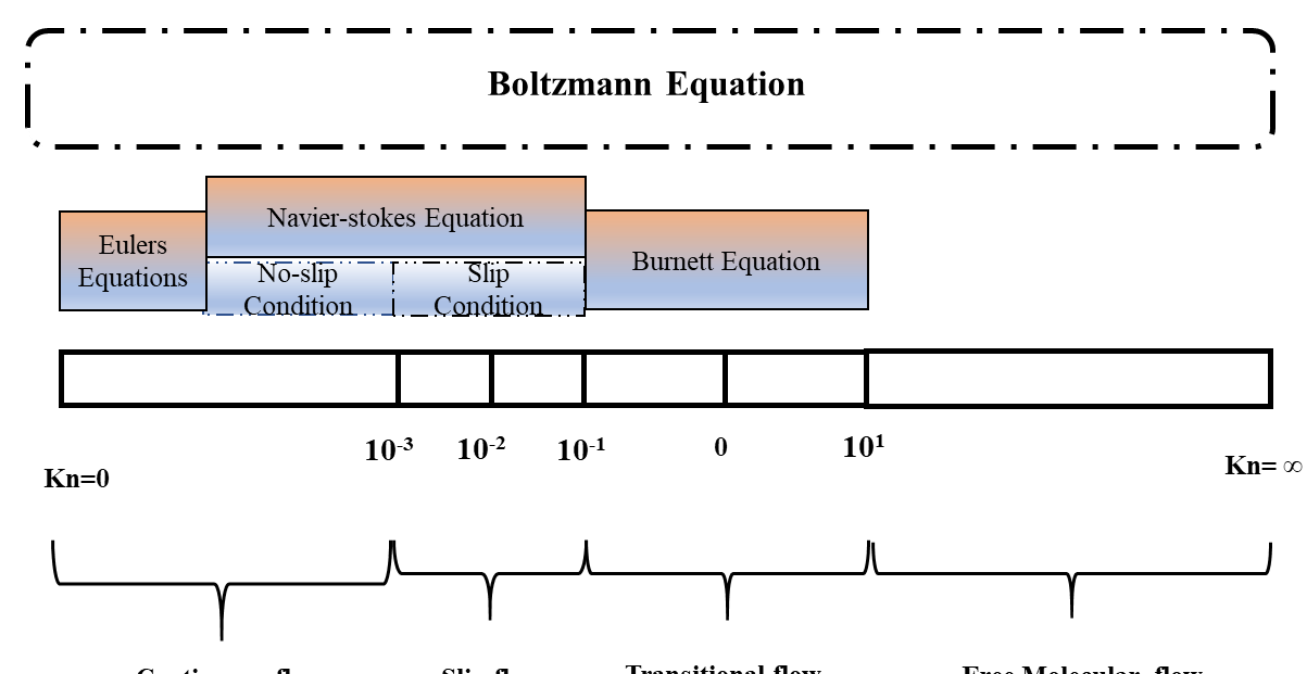

$\begin{array}{llll}\text { Continuum flow } & \text { Slip flow } & \text { Transitional flow } & \text { Free Molecular flow }\end{array}$

Figure 2.13 Flow regimes of gas in a microchannel based on Knudsen number (adopted from [74])

Table 2.4 Flow regimes of air through channels of different dimensions

\begin{tabular}{|l|l|l|l|l|}
\hline \multicolumn{5}{|c|}{ Channel Dimension in $\mu \mathrm{m}$} \\
\hline \multirow{3}{*}{ Gas } & $\begin{array}{l}\text { Continuum } \\
\text { Flow } \\
\text { (in } \mu \mathrm{m})\end{array}$ & $\begin{array}{l}\text { Slip Flow } \\
\text { (in } \mu \mathrm{m})\end{array}$ & $\begin{array}{l}\text { Transition } \\
\text { Flow } \\
\text { (in } \mu \mathrm{m})\end{array}$ & $\begin{array}{l}\text { Free Molecular } \\
\text { Flow } \\
\text { (in } \mu \mathrm{m})\end{array}$ \\
\hline Air & $>67$ & $0.67-67$ & $0.0067-0.67$ & $<0.0067$ \\
\hline
\end{tabular}

\subsection{Conclusion and Summary}

The literature has shed light on the microchannels and their potential in MEMS technology and how they are crucial for thermal pump performance. The existing artificial nose technology works primarily on mechanical pumps to inject the gas sample into the sensor array for identification. Thermal pump-based sniffing is applied for micro gas chromatography, which is not equivalent to the electronic nose as it can only detect single gas components. There is a need for MEMS-based microchannel sniffers as they can be portable and cost-efficient, along with the potential to be used on the field. In conclusion, MEMS-based microchannel sniffers that work on the thermal pump have not been explored previously and have high potential to serve as novel sniffers for electronic nose systems. 


\section{CHAPTER 3 \\ MECHANISM OF OPERATION OF THERMAL TRASNPIRATION EFFECT IN MICROCHANNEL}

\subsection{Introduction}

MEMS microchannel is a candidate technology for gas transport in devices such as micro-heat exchanges, air separation units, lab on chip [78]. In this work, the characteristics of gas flow and heat transfer in a microchannel need to be studied in order to properly design the device that allows the passage of a controlled volume of fluid over a sensor. Since the dimensions of the channel are in few tens to hundreds of micrometres, the term "microchannel" has become an accepted classifier for engineering which is associated with processes at this scale [30]. However, as the channel size becomes smaller, the gas flow in a channel will transform from no-slip to slip at the walls which has to be analysed. The fluid flow in a channel with smaller diameter may be subjected to a deviation from continuum assumption of gas flows, influence of external forces such as electrokinetic force, pressure force, gravitational force and uncertainty of the empirical factors including entrance and exit loss of coefficient for fluid. Hence, the theories and assumptions predicted in a conventional channel for gas flow and heat transfer will not be adequate for the microchannel [79].

In order to model the gas flow in a MEMS microchannel, it is required to consider the change in the gas density. The effects of change in gas density are generally referred to as rarefaction and compressibility effects. They are quantified in terms of non-dimensional parameters, Knudsen number (Kn) and Mach number (Ma) [80]. Thermal transpiration is a rarefaction effect which triggers the gas flow by the tangential temperature gradient along the channel surface. The factors that cause the thermal transpiration phenomenon are rarefaction effects, the compressibility of the fluid, velocity slip and heat transfer in the walls, and the gas's nature [81]. Chapter 3 discuss about the gas flow in the microchannel and heat transfer in the microchannel. 


\subsection{Heat Source Operating Conditions}

The microheater is assumed to be placed at the outlet of the microchannel. It placed in such a way that a uniform temperature is generated to the entire outlet of the microchannel. The heater is designed to operate at a maximum temperature of $70^{\circ} \mathrm{C}$. The initial time taken by the microheater to reach maximum temperature of $70^{\circ} \mathrm{C}$ is neglected because the sensor is assumed to be turned off. The sensor is turned on when the temperature of the microheater is $70^{\circ} \mathrm{C}$. The maximum temperature of $70^{\circ} \mathrm{C}$ is selected because it is considered as the feasible temperature of the microheater.

\subsection{Basic Principle and Mechanism of Operation}

The gas flow in a microchannel depends on the properties of the gas such as the molecular spacing between the gas molecules, the molecular diameter of the gas molecules, the mean free path of the gas molecules, the compressibility of the fluid and the wall effects.

\subsubsection{Microchannel Design and Principle}

Microchannels are usually made of materials with low thermal resistance in order to enhance the heat removing properties of the fluid. Their design also considers nature of the fluid [82], which in this work is air. Therefore, the microchannel should be designed such that it falls within the slip flow regime which is in the range of $0.001<\mathrm{Kn}<0.1$ because the rarefaction effect that causes Thermal transpiration flow becomes significant. In order to design the microchannel to fall under slip flow regime the parameters to be considered are the thermal conductivity of the material, thickness of the microchannel, the molecular diameter and spacing of the gas molecules at room temperature. In modelling a gas flow in MEMS microchannel, the continuum model and assumptions of thermodynamic equilibrium start to breakdown as the size of the channel is decreased.

\subsubsection{General Properties of Gas Flow}

For a diluted gas, the intermolecular interaction is binary collision where the molecules collide with another molecules. However, for a dense gas the rarefaction effect is compromised [83]. When the ratio of molecular spacing and diameter of the fluid is less than 1 then the fluid is said to be dilute. For a rarefied gas flow, since the gas density is 
low, the mean free path can increase up to or even higher than the macroscopic scale length of the channel, and the mean molecular spacing becomes larger than the molecular diameter $\delta>>\mathrm{d}$ [84]. In order to determine whether the fluid is dense or dilute, the mean molecular spacing $(\delta)$ of the fluid (air) and the diameter of the gas molecules has to be considered such that the condition in equation 3.1 is satisfied [84],

$$
\frac{d}{\delta} \ll 1
$$

where $\mathrm{d}$ is the diameter of the gas molecule and $\delta$ is the molecular spacing between the gas molecules.

The number density is an intensive quantity that describes the degree of concentration of countable molecules present in a chosen volume which is shown in Figure 3.1. The arrow near gas molecules represents the direction in which it is moving. The unit of number density is $\mathrm{m}^{-3}$. The gas molecules comprises of single gas species with same structured molecules. The number density is given by the equation 3.2.

$$
n=\delta^{-3}
$$

Thus, the mean free path depends on the diameter of the gas molecules and the number density. Mean free path $(\lambda)$ is the average distance travelled by the molecules between two successive collisions and it is given by the equation 3.3,

$$
\lambda=\frac{1}{\sqrt{2} \pi n_{v} d^{2}}
$$

where $\lambda$ is the mean free path, $n_{v}$ is the number density and $\mathrm{d}$ is the molecular diameter of the gas molecules. The number density can be calculated using the Avogadro's number $\left(N_{A}\right)$ and Ideal gas law. The equation 3.3 can be re-written as given by the equation 3.5,

$$
n_{v}=\frac{n N_{A}}{R T / P}
$$

where $\mathrm{n}$ is number of moles 


$$
\lambda=\frac{\mathrm{R} * \mathrm{~T}}{\sqrt{2} \pi \mathrm{N}_{\mathrm{A}} d^{2}}
$$

where $\mathrm{R}=$ universal gas constant $=8.3145 \mathrm{~J} / \mathrm{mol} \mathrm{K}, \mathrm{N}_{\mathrm{A}}=$ Avogadro's number $=6.0221$ $\times 10^{23} / \mathrm{mol}$.

For air in standard condition (STP) that is at a temperature of $273.15 \mathrm{~K}$ and pressure of $\mathrm{P}=1.013 \times 0^{5} \mathrm{~Pa}$, the molecular diameter $(\mathrm{d})$ is $0.37 \mathrm{~nm}$, molecular spacing $(\delta)$ is $3.3 \mathrm{~nm}$, the density number is $2.7 \times 10^{25} \mathrm{~m}^{-3}$, and the mean free path is $65 \mathrm{~nm}$ [84]. The characteristic length of the control volume should be greater than the mean free path of the air so that the flow of the gas molecule can be modelled by continuum approach.

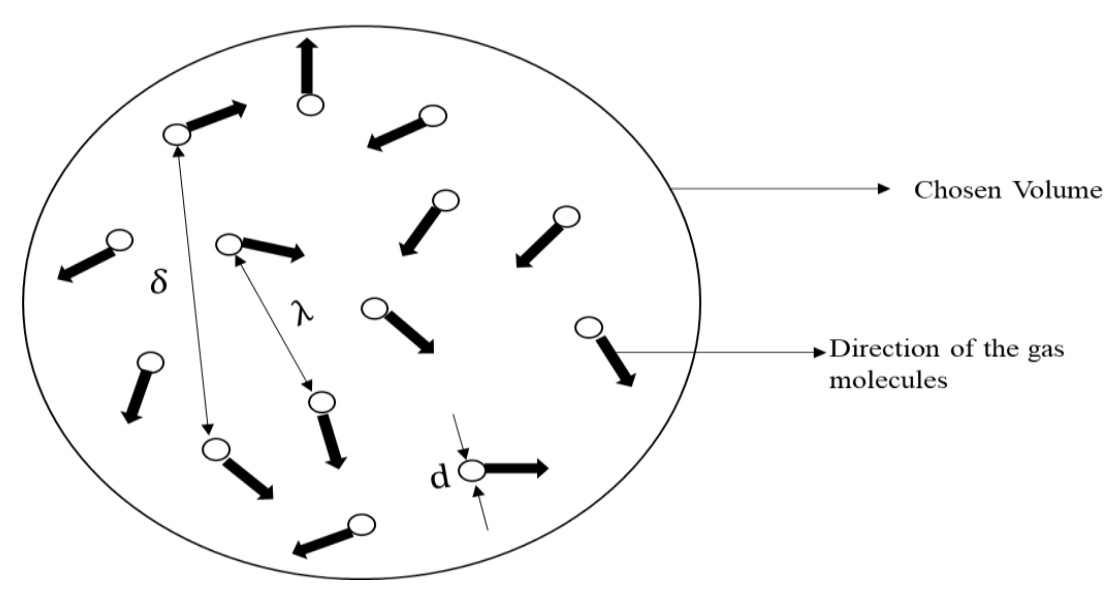

Figure 3.1 Mean free path, diameter, molecular spacing of gas molecules in a chosen volume

\subsubsection{Microchannel Design and Material}

The investigation of microchannel design and principle presented in the section 3.3.2 indicates that besides $\mathrm{Kn}$, there are other influencing parameters such as the material of the microchannel and the temperature of the heat source that have to be considered while modelling the gas flow. However, in order to precisely differentiate the gas flow regimes the $\mathrm{Kn}$ classification is used [85]. For a microchannel the Kn should fall in the range of $0.001<\mathrm{Kn}<0.1$ in order to trigger the thermal transpiration effect. In this work, two microchannels are investigated with cylindrical and rectangular geometries in order to 
understand the influence of the geometry in the flow of air by thermal transpiration effect. The dimension of the cylindrical and rectangular microchannels are designed to be $60 \mu \mathrm{m}$ in hydraulic diameter and $1 \mathrm{~cm}$ in length with $10 \mu \mathrm{m}$ thickness. The temperature is set to maximum of $70^{\circ} \mathrm{C}$ because it is considered as the maximum feasible temperature of the microheater. The dimension is designed to remain within the slip flow regime and discussed in chapter 4 . The microchannels are commonly made of silicon as it is the cost effective and widely used MEMS substrate that allows micromachining. Apart from silicon, in this work, pyrex is also considered which is another candidate material in micromachining. These materials have low thermal conductivity and high thermal difference. The thermal conductivities of the materials investigated in this work are listed in Table 3.1 [86]-[88].

Table 3.1 Materials and Thermal Conductivity

\begin{tabular}{|l|l|}
\hline \multicolumn{1}{|c|}{ Material } & \multicolumn{1}{c|}{$\begin{array}{c}\text { Thermal conductivity } \\
\text { at room temperature W/m.K }\end{array}$} \\
\hline Silicon & 130 \\
\hline Pyrex & 1.143 \\
\hline
\end{tabular}

\subsubsection{Wall Effects in The Microchannel}

Microchannel inner walls play a crucial role in determining the gas flow. In order to understand the heat transfer, it is necessary to investigate the velocity and temperature of the gas in the designed microchannel. The wall-adjacent layer effects are incorporated into the continuum-based mathematical model by imposing values of the variable viscosity along the microchannel walls and thermal conductivity of the material close to the channel walls, for solving the momentum and energy conservation equations [89], [90]. The velocity slip and the temperature jump occur when the momentum and energy is transferred between the colliding gas molecules impinging on the surface of inner walls. Increasing surface roughness increases the heat transfer performance, however it introduces flow resistance, which makes the friction coefficient rise sharply. As a result, surface roughness has a great influence on the flow and heat transfer performance. Hence, to model a gas flow in a microchannel within a slip flow regime to trigger the thermal transpiration effect, the 
slip boundary conditions have to be taken into account which will be discussed in section 3.7 .

\subsubsection{Compressibility Effect in The Fluid}

The term compressibility means the pressure or temperature changes due to a flow which is large enough to cause significant density change. The Mach number is used to measure the flow compressibility of the fluid. Mach number is defined as the ratio of the velocity of the fluid to the speed of sound in the fluid [91].

$$
M a=\frac{u}{c}
$$

where $\mathrm{u}$ is the velocity of the fluid and $\mathrm{c}$ is the speed of the sound in the fluid.

If the Mach number is less than 0.3, then the flow is said to be incompressible. If the Mach number is greater than 0.3, the flow is said to be compressible. Gases are generally considered as compressible while liquids are considered as incompressible. A gas flow in a microchannel by thermal transpiration effect happens when air creeps from a cold side to a hotter side due to the temperature gradient. The velocity of air and the Reynolds number (refer to section 4.4) defines that the flow is stokes flow. The velocity is in the range of $\mathrm{mm} / \mathrm{s}$. Hence, the density and pressure on air inside the microchannel does not change significantly because of the meagre volume of air. Thus, the air flow is considered as incompressible [92], [93].

\subsection{Fluid Flow Analysis Using Navier Stokes Equation}

Navier-Stokes equation can determine the velocity of the fluid flow in ducts, pipes, and channels. It is derived from Newton's second law of motion. The Navier-Stokes equations can be derived from conservation of momentum and continuity equations applied to properties of fluids. The Navier-Stokes equations are represented in the form of equation of states which are associated with the pressure, temperature, and density of the fluid [94][97]. 
Newton's second law of motion states that the force acting on the body is directly proportional to the mass of the body and acceleration of the body.

$$
\vec{F}=m \vec{a}
$$

The acceleration term in the equation 3.7 is the time derivative of velocity. Hence, equation 3.7 can be written as equation 3.8 .

$$
\sum \vec{F}=m \frac{d \vec{u}}{d t}
$$

The term mass in the equation 3.8 refers to the total mass entering and leaving the system in a controlled volume which means the mass is conserved and it can be represented as mass per unit volume, which is the density $(\rho)$ of the fluid. Hence, equation 3.8 can be rewritten as equation 3.9 .

$$
\sum \vec{F}=\rho \frac{d \vec{u}}{d t}
$$

The velocity is the change in acceleration with time. In fluid, there are two types of accelerations: a temporal acceleration and a convective acceleration. A temporal acceleration is the rate of change of velocity per time at a given point. The convective acceleration is the rate of change of velocity due to change in position of the fluid particles in a fluid flow. Hence, the mass and acceleration term are written as equation 3.10.

$$
\sum \vec{F}=\rho\left(\frac{\partial \vec{u}}{\partial t}+\vec{u} . \nabla \vec{u}\right)
$$

where $\rho$ is the density of the fluid, $\overrightarrow{\mathrm{u}} . \nabla \vec{u}$ is the of the convective acceleration of the fluid, $\frac{\partial \vec{u}}{\partial t}$ is the temporal acceleration of the fluid and $\nabla$ is the nabla operator or divergence which is a vector quantity. 
The force acting on the fluid in a controlled volume is given in equation 3.1. When a fluid flows through a confined geometry it exhibits viscous force due to shear stress at the fluid and solid boundary and the pressure force acting on the fluid.

$$
\text { Force }=\text { Surface force }+ \text { body force }
$$

The surface force is the pressure force and viscous force acting on the fluid and the body force is the external forces such as gravitational force, and electrokinetic force,

$$
\sum \vec{F}=-\nabla \mathrm{p}+\nabla \cdot \tau
$$

where $\nabla \mathrm{p}$ is the pressure force acting on the fluid and the negative sign denotes compression. $\nabla . \tau$ is the viscous force acting on the fluid.

The viscous force is represented by stress tensor which has nine components, defined as $\tau(x, y, z)$ in the cartesian plane shown in Figure 3.2. The viscous force acting within the fluid and it is given by Newton's law of viscosity. The shear stress between the fluid layers is proportional to velocity gradient of the fluid, given by the equation 3.12 ,

$$
\tau_{x y}=\mu\left(\frac{\partial u}{\partial y}+\frac{\partial v}{\partial x}\right)
$$

where $\tau$ is the shear stress, $\mu$ is the viscosity of the fluid, $\frac{\partial u}{\partial y}$ and $\frac{\partial v}{\partial x}$ are the velocity gradient of the fluid.

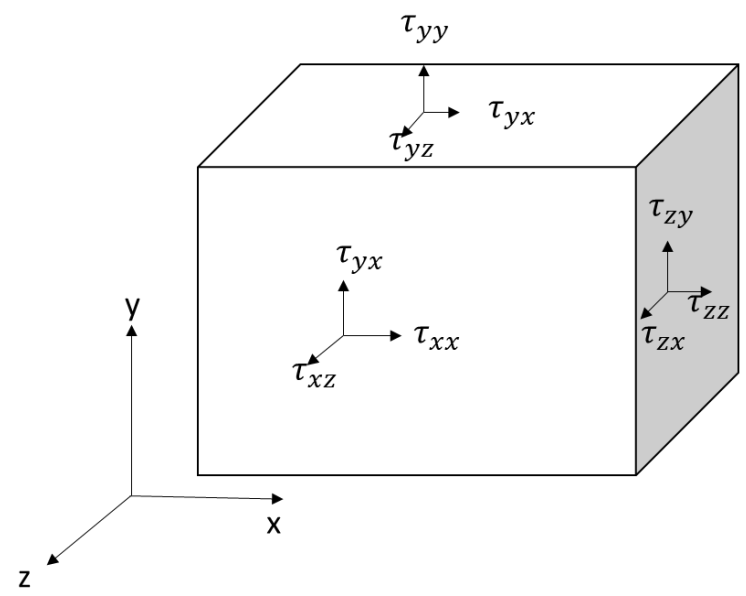

Figure 3.2 The nine components of tensor 
On applying the equation 3.12 on 3.10 and by implying constitutive relation between shear stress of the fluid flow and shear rate of the fluid under stress (Newton's Law of Viscosity) equation 3.13 is given by

$$
\sum \vec{F}=-\nabla p+\nabla \cdot(\mu \nabla \vec{v})
$$

The equation 3.13 can be re-written as

$$
\sum \vec{F}=-\nabla \mathrm{p}+\left(\mu \nabla^{2} \overrightarrow{\mathrm{v}}\right)
$$

Thus, from equation 3.10 and 3.14 we obtain the volumetric forces acting on the fluid in a closed volume. The momentum equation is represented as,

$$
\rho\left(\frac{\partial \vec{u}}{\partial t}+\vec{u} . \nabla \vec{u}\right)=-\nabla p+\left(\mu \nabla^{2} \vec{v}\right)+\vec{F}
$$

where $\vec{F}$ is the external force acting on the fluid.

Equation 3.15 has three variables for velocity $(\mathrm{x}, \mathrm{y}, \mathrm{z})$ and one variable for variable for pressure [97]. In order to solve the equation mathematically continuity equation is used which is given by equation 3.16 .

$$
(\nabla \cdot \overrightarrow{\mathrm{u}})=0
$$

In order to solve the Navier-Stokes equation in a microchannel, velocity slip and temperature jump boundary conditions are used. From 3.15 the velocity of the fluid at a particular point can be solved. However, in a rarefied gas flow, there is a tangential temperature gradient which induces the gas flow by a phenomenon called thermal transpiration. The convective heat transfer from the solid boundary to the liquid boundary is solved using energy equation described in the section 3.5.

\subsection{Temperature Distribution in Microchannel Using Energy Equation}

The Energy equation denotes the conservation of energy in a fluid flowing in a confined geometry. The energy equation is derived from first law of thermodynamics. According to the first law of thermodynamics the internal energy of a closed system is 
equal to the amount of heat supplied to the system minus work done by the system [98] given by equation. 3.17 .

$$
\begin{gathered}
\left(\begin{array}{c}
\text { Rate of change of } \\
\text { energy inside a fluid }
\end{array}\right)=\left(\begin{array}{c}
\text { Net flux of heat in } \\
\text { the system }
\end{array}\right)-\left(\begin{array}{c}
\text { Work done on the element by } \\
\text { surface and body forces }
\end{array}\right) \\
\Delta U=Q-\mathrm{W}
\end{gathered}
$$

The internal energy equation is written as in terms of scalar transport equation because the fluid is being moved due to temperature gradient. The transport equation is also called as convection-diffusion equation. So, the equation is written as equation 3.18 [98],

$$
\frac{\partial(\rho e)}{\partial t}+\nabla \cdot(\rho U e)=-\nabla \cdot q+S_{e}
$$

where $\frac{\partial(\rho e)}{\partial t}$ is the unsteady term characterized as internal energy, $\nabla .(\rho U e)$ is the diffusive term, $\mathrm{U}$ is the velocity of the particles which is being transported. The velocity term is derived from Navier-Stokes equation (3.15), $\nabla . q$ is the diffusion of the internal energy and $S_{e}$ is the external source of internal energy.

In order to get the temperature from the internal energy equation. The internal energy (e) can be written as equation 3.19 ,

$$
e=C_{p} T
$$

where $C_{p}$ is the specific heat capacity and $\mathrm{T}$ is the temperature field.

From substituting the equation 3.19 in 3.18 we get

$$
\frac{\partial\left(\rho C_{p} T\right)}{\partial t}+\nabla \cdot\left(\rho U C_{p} T\right)=-\nabla \cdot q+S_{e}
$$

By applying Fourier's law, we know that the heat flux is proportional to the negative gradient of the temperature field. The negative sign denotes the temperature from hot to cold. It is given as equation 3.21, 


$$
q=-K \nabla T
$$

where $\mathrm{K}$ is the coefficient of thermal conductivity of the material and $\nabla T$ is the temperature gradient.

Now, by substituting the equation 3.21 in 3.20 we get,

$$
\frac{\partial\left(\rho C_{p} T\right)}{\partial t}+\nabla \cdot\left(\rho U C_{p} T\right)=\nabla \cdot(K \nabla T)+S_{e}
$$

Equation 3.22 is the temperature equation or the internal energy equation. The unsteady

term $\left(\frac{\partial\left(\rho C_{p} T\right)}{\partial t}\right)$ denotes the rate of change of temperature of the moving liquid. The convection term $\left(\nabla \cdot\left(\rho U C_{p} T\right)\right)$ denotes the rate of change of velocity of the fluid which is derived from the momentum equation or Navier-Stokes equations. The diffusion term $(\nabla .(K \nabla T))$ is in the form of conduction as we have the coefficient of thermal conductivity $(\mathrm{K})$ of the material.

\subsection{Knudsen Layer Formation in The Microchannel}

The Knudsen layer is a kinetic layer which is formed when a fluid is flowing over solid boundary as shown in Figure 3.3 [99]. Even for a small $\mathrm{Kn}$, there is a domain adjacent to the wall of the channel in which the gas is out of equilibrium. The domain is considered as Knudsen layer which is in the order of mean free path. However, in a continuum flow regime the influence of the Knudsen layer is negligible because the mean free path is significantly smaller than the hydraulic diameter of the channel. The effect of the Knudsen layers must be taken into account in the slip flow regime where the Kn ranges from $0.001<\mathrm{Kn}<0.1$ because the number of gas molecules colliding with the wall becomes significant. But, in a slip flow regime the thickness of the Knudsen layer is small and can be neglected by providing boundary condition which are expressed as velocity slip and temperature jump at the walls. This is explained in detail in section 3.7. However, for high Knudsen number, that is when $\mathrm{Kn}>1$, the Knudsen layer dominates the flow passage of the channel where the intermolecular collision between the gas molecules is negligible and the interaction between the walls of the channel and gas molecules becomes significant. This happens in the transitional regime and free molecular regime where the Knudsen number range is $1<\mathrm{Kn}<10$ and $\mathrm{Kn}>10$. From Figure 3.3 it is seen that the actual slip 
velocity is smaller than the slip velocity obtained using Navier-Stokes equations with first order slip boundary conditions [99]. Hence, the microchannels in this work are designed within the slip flow regime where the Knudsen layer thickness is small when compared with free molecular regime. The boundary conditions in section 3.7 are used to solve the velocity in the Knudsen Layer.

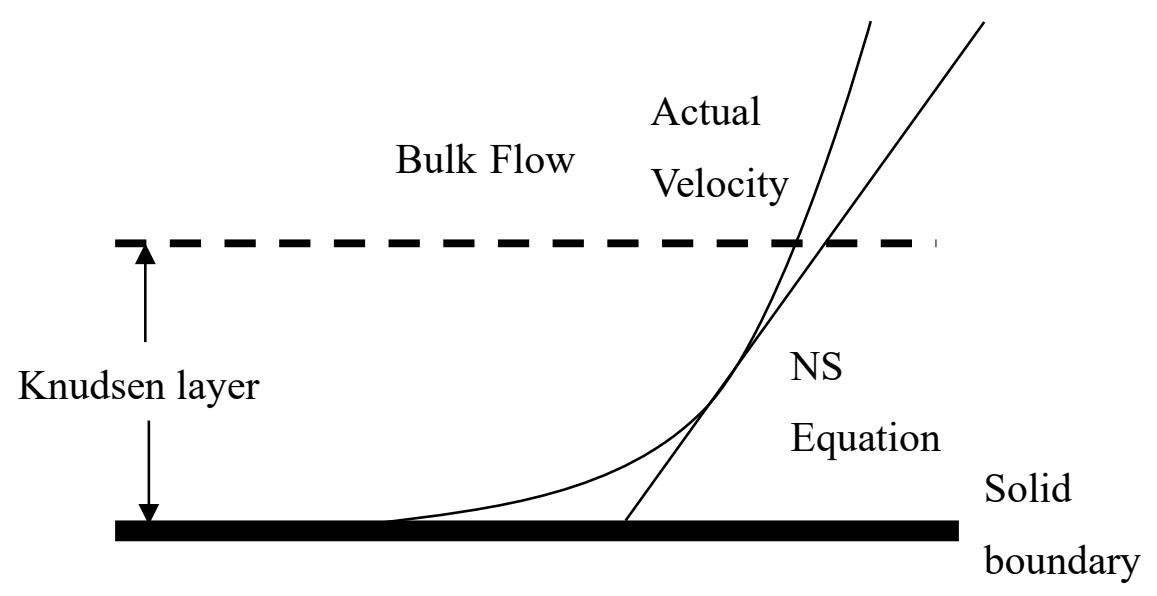

Figure 3.3 Schematic diagram of Knudsen layer (adopted from[99])

\subsection{Boundary Conditions to Determine The Fluid Flow}

The formation of Knudsen layer in the microchannel causes the Navier-Stokes equation to fail because the equations are valid when the gas molecules collide with another gas molecules. In order to solve the fluid velocity in the Knudsen layer, the boundary conditions are implemented which is discussed in section 3.7.2 and 3.7.4.

\subsubsection{Introduction to Boundary Conditions}

The flow regimes in a microchannel are classified based on the Kn and hydraulic diameter of the channel. The slip flow regime falls under the Kn range of $0.001<\mathrm{Kn}<$ 0.1 . The boundary conditions are used in a slip flow regime because in the Knudsen layer the collision of gas molecules are not adequate to reach the quasi thermodynamicequilibrium condition which causes the Navier-Stokes equation fail to explain the gas slippage in the walls of the microchannel [100]. The actual velocity within the Knudsen layer is smaller than the macroscopic slip velocity derived from the Navier-Stoke solutions shown in Figure 3.3. Thus, the first order slip boundary condition which includes Maxwell 
slip boundary and Smoluchowski temperature jump boundary conditions should be used for gas flows within $\mathrm{Kn}<0.1$. When the $\mathrm{Kn}$ increases, the higher-order boundary condition is required to capture the slip-velocity at the walls.

\subsubsection{Maxwell Velocity Slip Boundary Condition}

Maxwell introduced the first order slip model which assumes that the velocity at the wall is equal to the velocity at the mean free path. The velocity at the wall is directly proportional to the Kn. The series of experiment conducted by Kundt and Warburg showed that the gas slip at the walls occurs in a microchannel when the hydraulic diameter is comparable to the mean free path of the intermolecular collision [100]. The slip velocity is written as,

$$
u_{\text {slip }}=u_{s}-u_{\text {wall }}=\left.\xi \frac{d u_{s}}{d n}\right|_{s}
$$

where $u_{s}$ is the velocity of the fluid, $u_{w a l l}$ is the velocity of the at the wall, $\xi$ is the slip length also called the coefficient of slip, $\mathrm{n}$ is the direction of the flow and s denotes the surface variable.

The aim of Maxwell's experiment is to derive a condition for the gas molecules which comes in contact with the solid boundary. In the slip flow regime, the collision between the wall and the gas molecules is considered and the intermolecular collision between the gas molecules is almost negligible. Maxwell proposed that for every unit area of the wall a fraction of gas molecules is absorbed by the surface due to wall roughness or condensation and evaporation process and it is remitted with the velocity corresponding to the gas and temperature at the wall whereas other fraction is perfectly emitted at the wall as shown in Figure 3.4. Hence a part of the gas molecules experiences specular reflection and other part of the gas molecules experience diffusive reflection as shown in Figure 3.4. The fraction of the molecules that are absorbed by the wall is given by Tangential Momentum Accommodation Coefficient $(\sigma)$ (TMAC) which is a dimensionless coefficient. Thus, the Maxwell slip boundary condition is given as [98], [99] equation 3.24, 


$$
u_{s}-u_{w}=\left.\frac{2-\sigma_{v}}{\sigma_{v}} \frac{\lambda}{\mu} \frac{\partial u_{s}}{\partial n}\right|_{w}+\left.\frac{3 \mu}{4 \rho T} \frac{\partial T}{\partial s}\right|_{w}
$$

where $u_{s}$ is the fluid axial velocity, $\mathrm{u}_{\mathrm{w}}$ is the surface axial velocity, $\sigma_{v}$ is the tangential momentum slip coefficient, $\lambda$ is the mean free path of the gas molecules and $\mu$ is the dynamic viscosity, $\rho$ is the density of the fluid and $\frac{\partial T}{\partial s}$ is the change in temperature with the surface of the wall. The first term on the right in equation 3.24 represents the slip due to the velocity gradient normal to the surface and the second term in equation 3.24 is due to the temperature gradient along the surface, also known as thermal transpiration.

Thus, the equation 3.24 is used to solve the velocity of the fluid at the solid boundary with the initial conditions such as temperature at the wall, viscosity of the fluid and the density of the fluid.

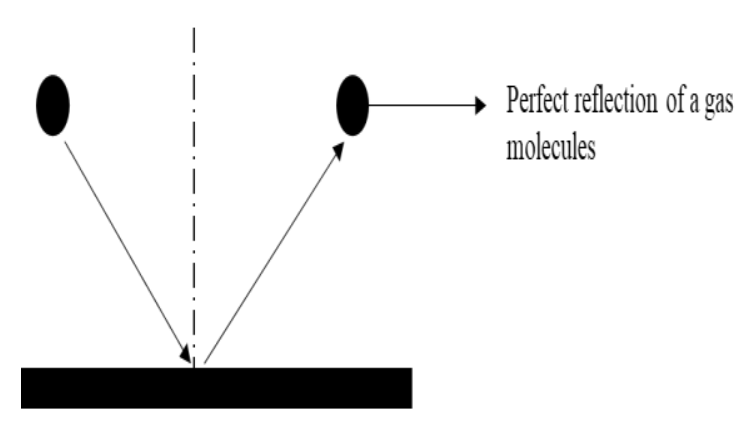

a

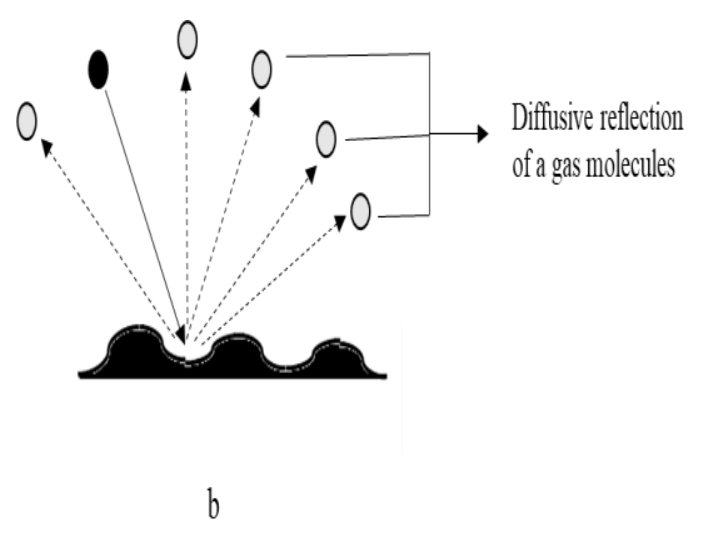

Figure 3.4 (a) Perfect (specular) reflection of a fraction of the molecules; (b) Absorption and diffuse reemission of a fraction of the molecules. The circle denotes the gas molecules impinging on the surface (adopted from [102])

\subsubsection{Assumption of Maxwell's Tangential Momentum Accommodation Co-efficient}

The value of tangential momentum accommodation coefficient TMAC is required while prescribing the boundary condition for flow of gases in the slip and transition flow regimes. The value of TMAC is dependent on a number of parameters including nature of the gas, pressure of the gas, material of the surface, surface cleanliness and roughness, and 
surface temperature. There are different approaches in measuring the TMAC which are as follows,

1) Oil drop experiment of Millikan [101]

2) Rotating cylinder method [102], [103]

3) Spinning rotor gauge method [104]

4) Molecular beam technique [105]

5) Flow through microchannels [106], [107]

6) Direct Simulation Mote Carlo Simulation [108]

Based on the literature, the TMAC value for diatomic gases like air over a silicon and pyrex smooth surface is in the range of 0.87-0.9 [109]-[111]. When $\sigma_{v}=1$ the gas molecules are reflected back from the wall with zero average tangential velocity resulting in diffusive reflection. Thus, the TMAC is assumed to be 0.9 resulting in diffusive reflection and the surface of the material is assumed to have some roughness surface.

\subsubsection{Smoluchowski Temperature Jump Boundary Condition}

Temperature jump occurs due to imperfect accommodation of internal energy in the gas-solid boundary which is analogous with gaseous slip. The temperature jump theory was developed by Smoluchowski which is developed from Maxwell slip velocity [112] . The distance of the temperature jump is proportional to the pressure of the fluid and it is equivalent to the mean free path of gas molecules. Thus, like Maxwell's slip velocity, the thermal accommodation coefficient $\left(\sigma_{t}\right)$ represents the fraction of gas molecules which are reflected and re-emitted and possess the same amount of heat energy that of the wall. It can be expressed as [113] given in equation 3.25,

$$
E_{i}-E_{r}=\sigma_{t}\left(E_{i}-E_{w}\right)
$$

where $E_{i}$ is the total energy of the incident molecules, $E_{r}$ is the total energy reflected and re-emitted molecules, $\sigma_{t}$ is the thermal accommodation co-efficient, $E_{w}$ is the energy of gas molecules emitted at wall temperature. 
The temperature jump occurs in a thermal transpiration flow. Since the rarefied gas flow which is triggered by the tangential temperature variations along the surface, the momentum and energy equations are coupled through the Thermal transpiration effects. Thus, at the wall the gas experiences a slip velocity and temperature jump that makes the gas move from cold to hot region which also results in pressure variation subjected to a tangential temperature gradient.

The incident and reflected energy is represented as temperature, which is given by equation $3.26[98]$,

$$
\begin{gathered}
E_{i}-E_{w}=\frac{k}{2} \frac{\partial T}{\partial n}+\frac{C_{v} p(1+\gamma)}{2 \sqrt{2 \pi R T}}\left(T_{o}-T_{g}\right) \\
E_{i}-E_{r}=k \frac{\partial T}{\partial n}
\end{gathered}
$$

where $\mathrm{k}$ is the thermal conductivity of the material, $C_{v}$ specific heat per unit volume, $\mathrm{p}$ is the pressure of the fluid, $\gamma$ is the ratio of specific heat, $R$ is the gas constant, $T$ is the absolute temperature, $\frac{\partial T}{\partial n}$ is the change in temperature at the surface of the solid and liquid boundary, $T_{o}$ is the temperature at the wall surface and $\mathrm{T}_{\mathrm{g}}$ is the temperature of the gas.

On rearranging the equations 3.26 and 3.27 , the temperature jump is represented as [98],

$$
T_{o}-T_{g}=\frac{2-\sigma_{t}}{\sigma_{t}} \frac{2 \gamma}{1+\gamma} \frac{k \lambda}{\mu C_{p}} \frac{\partial T}{\partial n}
$$

where $C_{p}$ is the specific heat capacity at the constant pressure, $\mu$ is the viscosity of the fluid.

The boundary conditions derived in equation (3.24) and (3.28) are applied in the Navier-Stokes equations. The Navier-Stokes equations fails in the Knudsen layer because of the absence of intermolecular collision of the gas molecules. The collision occurs between the gas molecules and the solid boundary. Hence, momentum and energy are exchanged between the gas molecules and the solid boundary. This leads to formation of a layer called Knudsen layer which is the thickness of mean free path of the gas molecules. In slip flow regime $(0.001<\mathrm{Kn}<0.1)$, the flow in the Knudsen layer cannot be analysed as 
continuum. Hence, the (3.20) and (3.24) boundary conditions are used to solve the velocity at the Knudsen layer.

\subsection{Conclusion and Summary}

In this chapter the properties of gas flow, basic physics involved in the gas flow and design of the microchannel are studied. The derivation of the governing equations, Navier-Stokes and Energy equation provide the understanding on the velocity, the direction of the fluid flow and heat transfer from the walls to the fluid in the microchannel. The slip velocity and temperature jump boundary conditions which are used to determine the fluid and the heat transfer near the microchannel walls are explained in this chapter. The dimensions of the microchannel and the fluid flow and heat transfer are discussed, but they need to be analysed for optimising the microchannel such that the gas flow passage of the microchannel falls within the slip flow regime. These are discussed in chapter 4. 


\section{CHAPTER 4 \\ CRITICAL DESIGN PARAMETERS AND AIR FLOW ANALYSIS IN THE PROPOSED MICROCHANNEL}

\subsection{Introduction}

The nature of the gas such as the molecular weight, molecular diameter (d), the molecular spacing between the gas molecules $(\delta)$, mean free path of the gas molecules $(\lambda)$, pressure of the gas molecules and temperature characterises the velocity flow rate due to rarefaction effect in the microchannel [114]. Hydraulic diameters ranging from 30-90 $\mu \mathrm{m}$ are considered to model a gas flow in cylindrical and rectangular microchannels with in the slip flow regime at a temperature of $70^{\circ} \mathrm{C}$ as mentioned in section 3.1. The analytical modelling can be used to calculate the velocity of the moving fluid in the microchannel by using the momentum equations. However, to couple the Energy equation and NavierStokes equations along with the boundary conditions, finite element analysis is performed. [115]. Thus, this chapter will focus on factors such as critical design parameters including the influence of hydraulic diameter of the cylindrical and rectangular microchannel, the impact of temperature, the impact of different material, the sniffing time, volume of air during the sniffing time and time taken for the channel to cool down at room temperature.

\subsection{Finite Element Analysis of Fluid velocity and Heat Transfer in Microchannel}

Finite element analysis simulates a predefined physical phenomenon using a numerical technique. The finite element analysis technique is used to study the different aspects of the design, such as discretization errors, rates of convergence and stability for different types of finite element approximation [116]. The flow diagram in Figure 4.1 shows the methodologies to approach a problem in finite element analysis. 


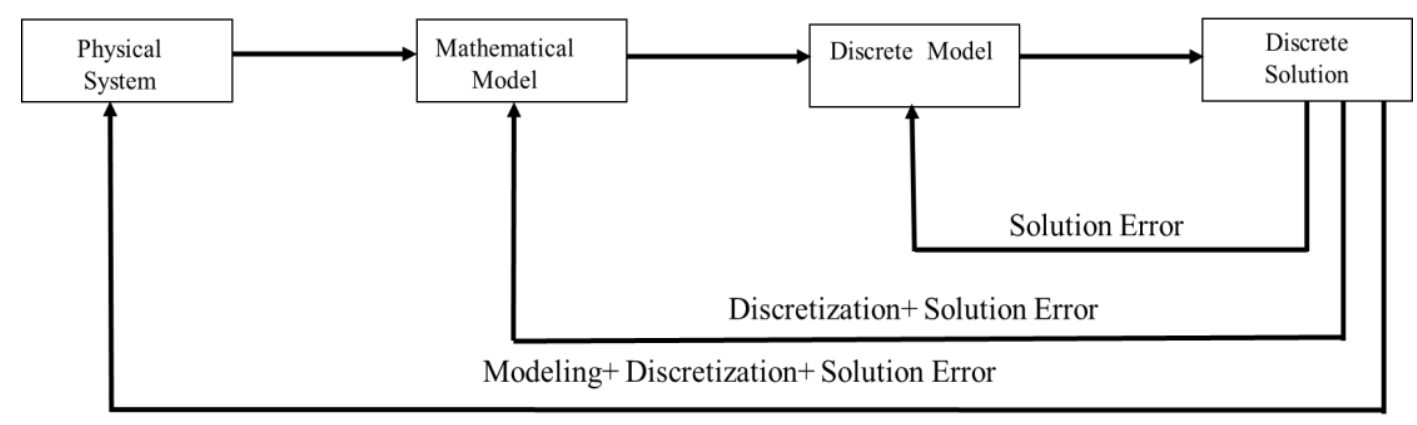

Figure 4.1 Main Stages of Finite Element Analysis [116]

In the case of microfluidics, where the fluid flow and heat transfer are coupled to solve the problem, the nature of the flow is calculated with change in time. So, a time-dependent study is performed to investigate the influence of temperature in the fluid velocity. Hence, the need to understand the flow as a function of time, the temperature change, which impacts the flow along with different material properties and the influence of the geometry can be modelled and investigated using the finite element analysis method.

The finite element analysis is used to calculate the velocity of the fluid which is triggered by the applied temperature. The analytical modeling can be used to calculate the velocity of the fluid using the slip boundary conditions by applying the Navier-stokes equation but in order to couple the heat transfer (energy equation) with the Navier-stokes equation, finite element analysis is implied to solve the problem. The temperature applied at the outlet of the microchannel causes the fluid to move from cold side to the hot side of the microchannel. The microchannel's wall thickness and the impact of the microchannel materials (silicon and pyrex) in determining the fluid velocity and temperature distribution in the entire microchannel volume are analysed using finite element analysis

\subsubsection{COMSOL Multiphysics}

COMSOL Multiphysics combines multiple physics-based environments by setting complex boundary conditions in multi-dimensional forms and build detailed geometrical structures for analysis [117]. The design and modelling of microelectromechanical systems (MEMS) at small length scales must consider the effects of several physical phenomena in their working principle and COMSOL software is ideally suited for MEMS application. The microfluidics module in COMSOL Multiphysics is used to model the cylindrical and 
rectangular geometry of the microchannel with materials such as silicon and pyrex. With the assistance of this comprehensive modelling tool, meshes can be built. The tool has a wide range of studies and options of post-processing the results with high precession.

The fluid flow in the microchannel is trigged by the thermal transpiration effect. For this reason, the Slip Flow interface involves the heat flow equations and the Navier-Stokes equation [118],[119]. In the microchannel, the slip flow rate is inversely proportional to the channel dimension [120].

\subsubsection{Meshing Characteristics of The Microchannel}

In COMSOL Multiphysics, after modelling the geometry, the next important step is to mesh the modelled geometry. Meshing is performed after assigning the physics to the respective geometry and adding the boundary conditions with appropriate loads (such as temperature, thermal insulation and slip wall) and constraints (such as open boundary, close boundary, inflow, and outflow condition). The meshing plays a significant role in how the geometry with boundary conditions is going to be solved. The mesh determines the number of elements and element-types (such as triangular, tetrahedral, and quadrilateral) that the geometry is divided, the size of each element, and the density of the elements. These factors determine the geometry's computation time, the space required for the model to be solved. The mesh can be divided into two types based on the sequence. They are physics-controlled and user-controlled mesh. The physics-controlled meshes are set with pre-defined meshing qualities such as extremely fine, extra-fine, finer, fine, normal, coarse, coarser, and extra coarser. The microchannel has a thickness of $10 \mu \mathrm{m}$ which causes the element size of the triangular finer mesh greater than the domain of the microchannel as shown in Figure 4.2. Thus, mesh warnings are generated shown in Figure 4.2 , and it is time consuming because of the element size and density. Thus, in order to get the results in the best possible minimal time, the user-controlled meshing characteristics of the COMSOL is conducted on the basic geometry of the cylindrical and rectangular microchannel. 
(a)

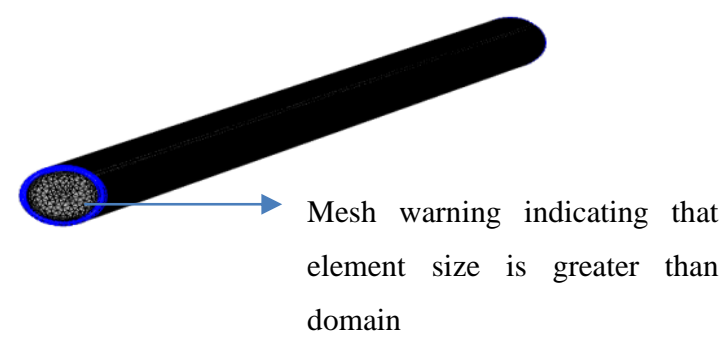

(b)

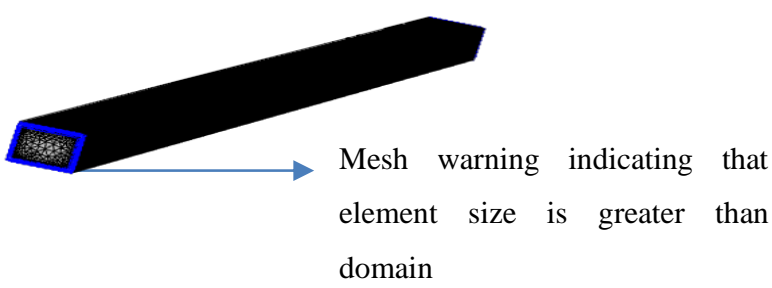

Figure 4.2 Finer meshing sequence of (a) cylindrical and (b) rectangular microchannel

The customised mesh is used to generate detailed meshing in the specific domain in the geometry where the fluid flow needs to be computed and generate a coarser mesh in the rest of the domain to reduce the number of elements while meshing. Since the microchannel does not have any deforming domain, the swept mesh is used to specify the distribution of the elements and specify a distribution sub-feature to the non-uniform element size in the thickness and the length of the microchannel [121]. Since the microchannel cross-section is uniform, the swept mesh is used to sweep from the inlet, which is the microchannel's source face, to the outlet, which is the destination face, by covering the entire domain of the microchannel as shown in Figure 4.3 [122]. 
(a)

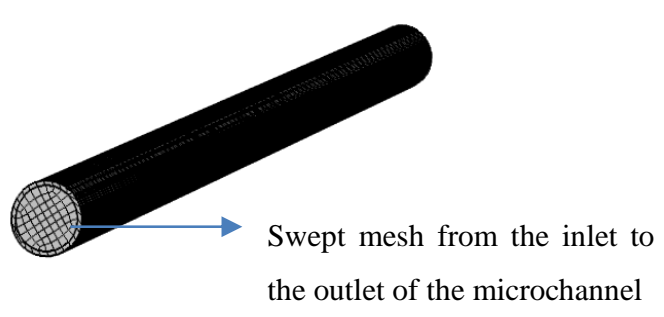

(b)

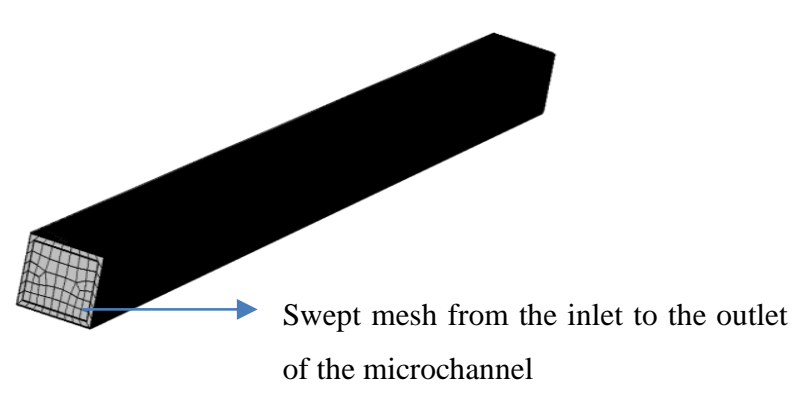

Figure 4.3 Swept meshing sequence of (a) cylindrical and (b) rectangular microchannel

The number of elements in the physics-controlled finer mesh is 2,975,245 whereas the number of elements in the user-controlled swept mesh is 104,000. Thus, the number of elements and computation time are reduced when compared with physics-controlled mesh. The parameters of the cylindrical and rectangular microchannel for mesh analysis are set in below Table 4.1 and 4.2 .

Table 4.1 Dimension of the Cylindrical Microchannel for mesh analysis

\begin{tabular}{|l|l|l|}
\hline Parameters & Value & Unit \\
\hline Length of the microchannel & 1 & $\mathrm{~cm}$ \\
\hline $\begin{array}{l}\text { Hydraulic Diameter of } \\
\text { microchannel }\end{array}$ & 60 & $\mu \mathrm{m}$ \\
\hline Thickness of the wall & 10 & $\mu \mathrm{m}$ \\
\hline
\end{tabular}


Table 4.2 Dimension of the Rectangular Microchannel for mesh analysis

\begin{tabular}{|l|l|l|}
\hline Parameters & Value & Unit \\
\hline Length of the microchannel & 1 & $\mathrm{~cm}$ \\
\hline $\begin{array}{l}\text { Hydraulic Diameter of } \\
\text { microchannel }\end{array}$ & 60 & $\mu \mathrm{m}$ \\
\hline Width of the microchannel & 72 & $\mu \mathrm{m}$ \\
\hline Breadth of the microchannel & 52 & $\mu \mathrm{m}$ \\
\hline Thickness of the wall & 10 & $\mu \mathrm{m}$ \\
\hline
\end{tabular}

\subsection{Analysis of Boundary Conditions in The Microchannel}

The boundary conditions are constraints given to model a fluid flow and heat transfer within the domain geometry. A boundary condition is set based on how the momentum and energy equation is solved which serves as the purpose of the simulation. By varying the boundary condition, the simulation results changes. The boundary conditions for the cylindrical and rectangular microchannel is shown in Figure 4.4.

(a)

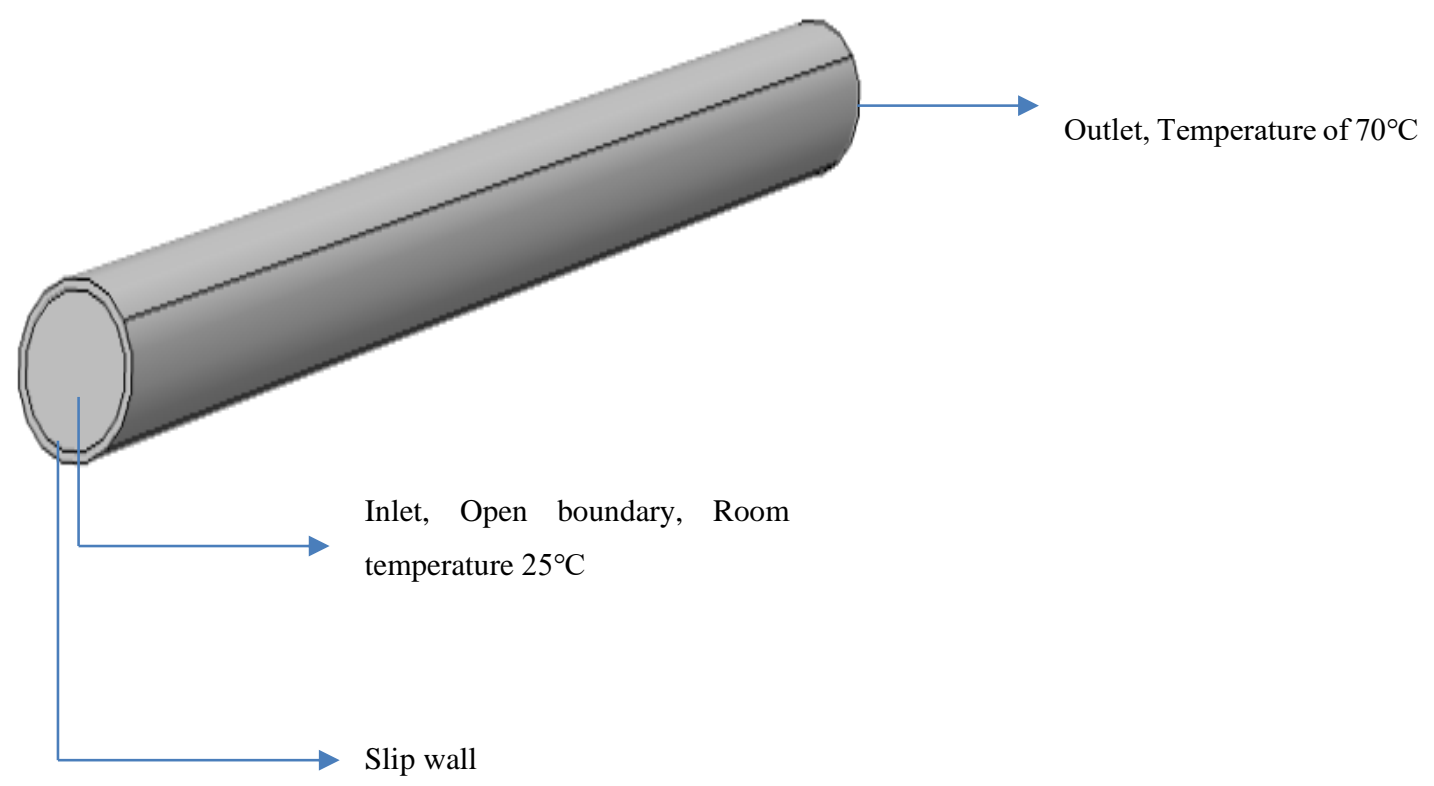


(b)

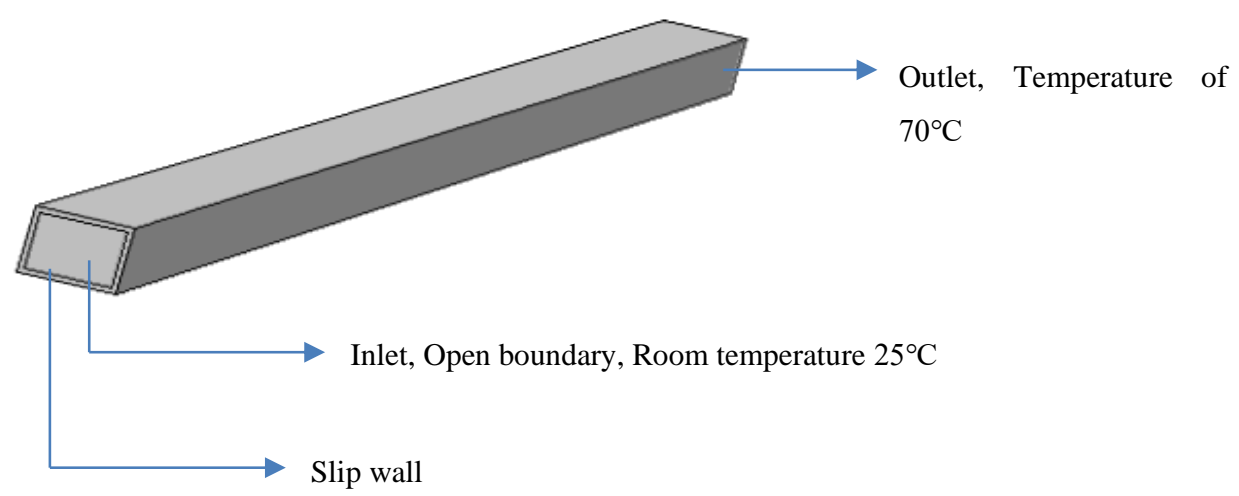

Figure 4.4 The boundary conditions of the (a) Cylindrical and (b)Rectangular Microchannel

\subsubsection{Open Boundary in The Microchannel}

The open boundary conditions depict the boundaries in the geometry, which are in contact with the large fluid volume. In an open boundary condition, the fluid can enter and exit the domain on the boundaries. The microchannels inlet as shown in Figure 4.4 are open boundary because it is exposed to an open environment to collect the sample. Hence, an open boundary is provided at the inlet.

\subsubsection{Outlet in The Microchannel}

The outlet boundary condition is used on boundaries where there is a net outflow from the domain. Unlike an open boundary, the outlet boundary condition will only allow the fluid to exit the domain and not enter the domain. The microheater is placed at the outlet of the microchannel (refer to section 3.2). The outlet of the microchannel is assumed to be connected with another chamber which comprises of an array of sensor. The air which is sniffed inside the channel is passed through the outlet and is delivered to the sensors which is positioned inside the chamber. The outlet boundary condition ensures that the air is delivered to the sensors in the chamber, and it is not allowed to enter the microchannel from the chamber. 


\subsubsection{Temperature Distribution on The Microchannel}

The temperature of $70^{\circ} \mathrm{C}$ is given at the outlet domain. The heater is assumed to be placed at the microchannel outlet, and it provides uniform heating all over the outlet boundary. A time-dependent study is performed, and the heater is assumed to generate maximum of $70^{\circ} \mathrm{C}$ at time $\mathrm{t}=0 \mathrm{~s}$ (refer to section 3.2). Thus, a uniform temperature is maintained at the outlet, and the temperature is distributed from the outlet to the microchannel's inlet. The temperature is maintained throughout the microchannel's surface and volume. The energy equation (refer section 3.5) is solved based on the input temperature $\left(70^{\circ} \mathrm{C}\right)$. A 3-D analysis is made as shown in Figure 4.5 to validate the heat flow with the function of time.

(a)

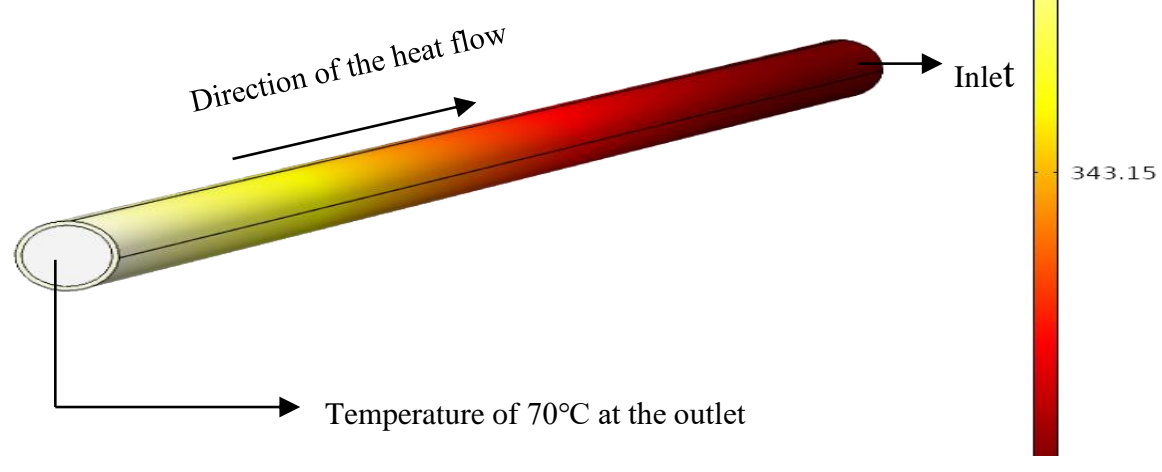

(b)

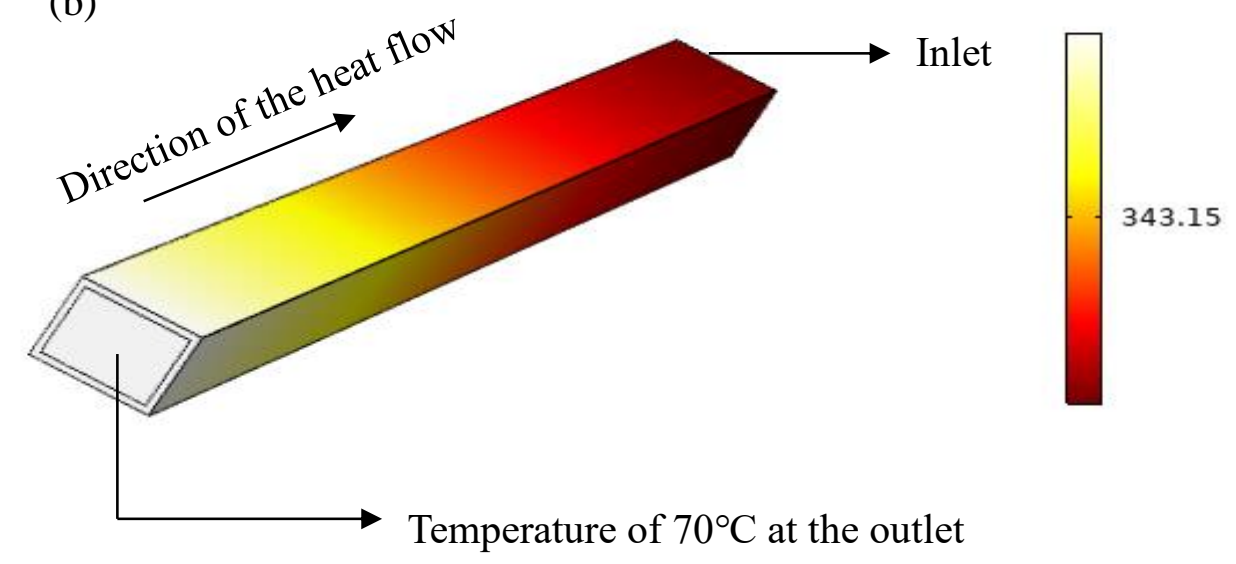

Figure 4.5 3-D Analysis of the temperature distribution in (a) Cylindrical (b) Rectangular microchannel 


\subsubsection{Slip Walls on The Microchannel}

In the microchannel boundary layer, the inner walls are given slip boundary conditions as shown in Figure 4.4. Maxwell's slip boundary condition and Von Smoluchowski temperature jump boundary conditions are applied at the microchannel's inner walls (refer to section 3.7.2 and 3.7.3). The boundary conditions solve the velocity and temperature jump of air in the Knudsen layer. In Maxwell's slip model, the TMAC $\left(\sigma_{v}\right)$ determines whether the gas molecules exhibit a diffusive reflection or specular reflection when they come in contact with the microchannel's inner wall. In COMSOL Multiphysics, TMAC is 0.9 by default which depicts the diffusive reflection. The TMAC value ranges from 0 to 1 . It depends on the fluid and the material of the microchannel. For silicon and pyrex material the TMAC of air ranges from 0.87 to 0.9 (refer to section 3.7.3). When $\sigma_{v}=$ 0 there is no momentum exchange between the fluid and microchannel's wall as a result the flow becomes inviscid and the viscous stress zero. Thus, resulting in specular reflection of the air molecules. When $\sigma_{v}=1$ the gas molecules are reflected back from the wall with zero average tangential velocity resulting in diffusive reflection. In diffusive reflection the momentum is exchanged between the gas molecules and the microchannel's wall [123]. Diffusive reflection is more likely to occur on a rough surface. The thermal accommodation coefficient is the energy that the gas molecules possess when they incident or reflect from the wall surface per unit time. The tangential momentum accommodation coefficient and thermal accommodation coefficient depend on the gas molecules, the surface roughness of the microchannel's inner walls, local pressure, and the gas flow direction [124].

\subsection{Reynolds Number in Microchannel}

Reynolds number is a dimensionless number that is used to categorise the nature of the flow. The Reynolds number defines the fluid system in which the fluid's velocity is controlled with the effect of the fluid's viscosity. Based on the Reynolds number, the flow is categorised as laminar, transition and turbulent flow, shown in Table 4.3. Reynolds number is the ratio of inertial forces to the viscous force that is exhibited in the fluid. The nature of the flow is proportional to the velocity and characteristic length of the microchannel. It is given by [125], 


$$
R e=\frac{\rho U L}{\mu}
$$

where, $\rho$ is the density of the fluid, $\mathrm{U}$ is the velocity of the fluid, $\mathrm{L}$ is the characteristic length and $\mu$ is the viscosity of the fluid.

Table 4.3 Classification of Flow based on Reynolds Number [125]

\begin{tabular}{|l|l|}
\hline Reynolds Number & Nature of the Flow \\
\hline $\operatorname{Re}<1$ & Stokes Flow \\
\hline $\operatorname{Re}=1$ to 2300 & Laminar Flow \\
\hline $\operatorname{Re}=2300$ to 4000 & Transitional Flow \\
\hline $\operatorname{Re}>4000$ & Turbulent Flow \\
\hline
\end{tabular}

In the microchannel, the fluid velocity is very slow, and the microchannel dimension is in the range of $\mu \mathrm{m}$. The velocity of the air reduces with increase in time. The maximum velocity of the cylindrical microchannel modelled using pyrex is $0.294 \mathrm{~mm} / \mathrm{s}$ at $\mathrm{t}=0 \mathrm{~s}$ and the velocity of the rectangular microchannel modelled using pyrex is 0.297 $\mathrm{mm} / \mathrm{s}$ at $\mathrm{t}=0 \mathrm{~s}$. The Reynolds number is calculated in COMSOL Multiphysics for rectangular and cylindrical microchannel modelled using pyrex material. Figure 4.6 depicts that the Reynolds number is less than 1 . When the Reynolds number is less than 1 , the flow is categorised as stokes or creeping flow [126]. In stokes flow, the viscous force dominates the inertial force because the fluid's velocity is streamlined. Thus, the inertia term in the Navier Stokes equation is neglected when dealing with stokes flow.

(a)

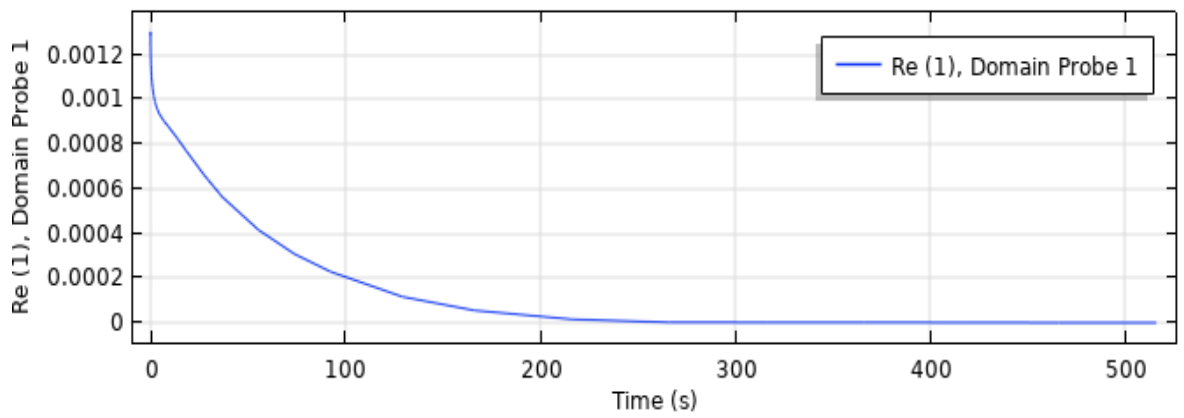


(b)

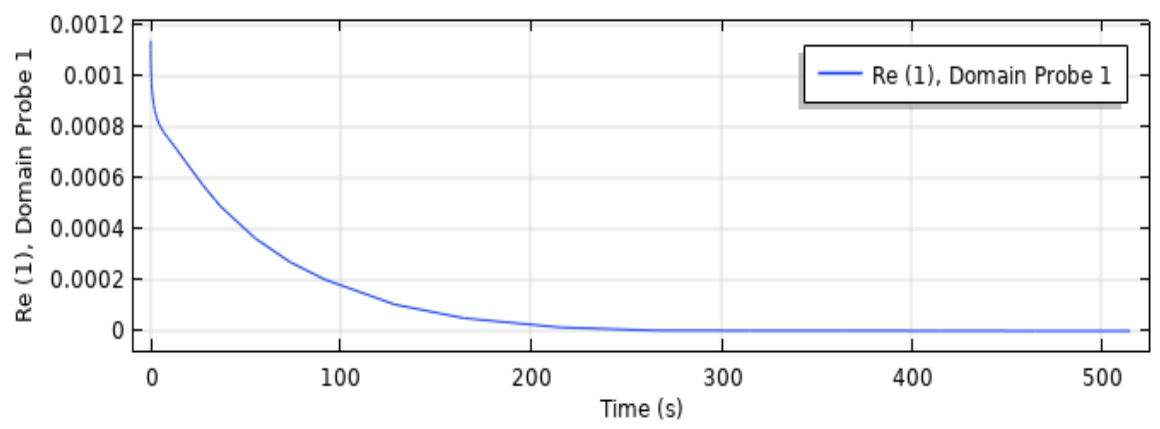

Figure 4.6 COMSOL simulation to indicate Reynolds number less than 1 for (a) Cylindrical Microchannel (b) Rectangular Microchannel

The Knudsen number can be related to the Mach number and Reynolds number. The relation is given by,

$$
K n=\frac{M a}{R e} \sqrt{\frac{\gamma \pi}{2}}
$$

where, Ma is the Mach number, Re is the Reynolds number and $\gamma$ is the ratio of specific heat. The Knudsen number can also be calculated using the equation (4.2).

\subsection{Investigation of Knudsen Number in The Microchannel}

In order to analyse the slip flow regime of air for a temperature of $70^{\circ} \mathrm{C}$ in the microchannel, hydraulic diameter of 30-90 $\mu$ m dimensions were modelled to study the $\mathrm{Kn}$ of the air molecules. The hydraulic diameters of 30-90 $\mu$ m based on Table 2.1 in section 2.2. The $\mathrm{Kn}$ is investigated based on the hydraulic diameter and the heat source at the microchannel's outlet (maximum of $70^{\circ} \mathrm{C}$ ) for the wall thickness of $10 \mu \mathrm{m}$. The mean free path for the investigated dimensions is $0.0769 \mu \mathrm{m}$ for analytical calculation and $0.079 \mu \mathrm{m}$ in COMSOL results. The analytical values do not vary significantly with COMSOL values. The Kn varies with the dimensions.

Figure 4.7 shows that the $\mathrm{Kn}$ increases with a decrease in the microchannel dimension for a temperature of $70^{\circ} \mathrm{C}$ because the $\mathrm{Kn}$ is directly proportional to the mean free path and inversely proportional to the hydraulic diameter. The $\mathrm{Kn}$ is 0.0026 for the 
hydraulic diameter of $30 \mu \mathrm{m}$ and 0.0009 for the hydraulic diameter of $90 \mu \mathrm{m}$. The Kn for the microchannel of hydraulic diameter of $60 \mu \mathrm{m}$ and $70 \mu \mathrm{m}$ are 0.0013 and 0.0011 . The hydraulic diameter of $70 \mu \mathrm{m}$ falls exactly on the slip flow regime of $\mathrm{Kn}=0.0011$. Hence, the hydraulic diameter of $60 \mu \mathrm{m}$ is chosen because it falls with in the slip flow regime.

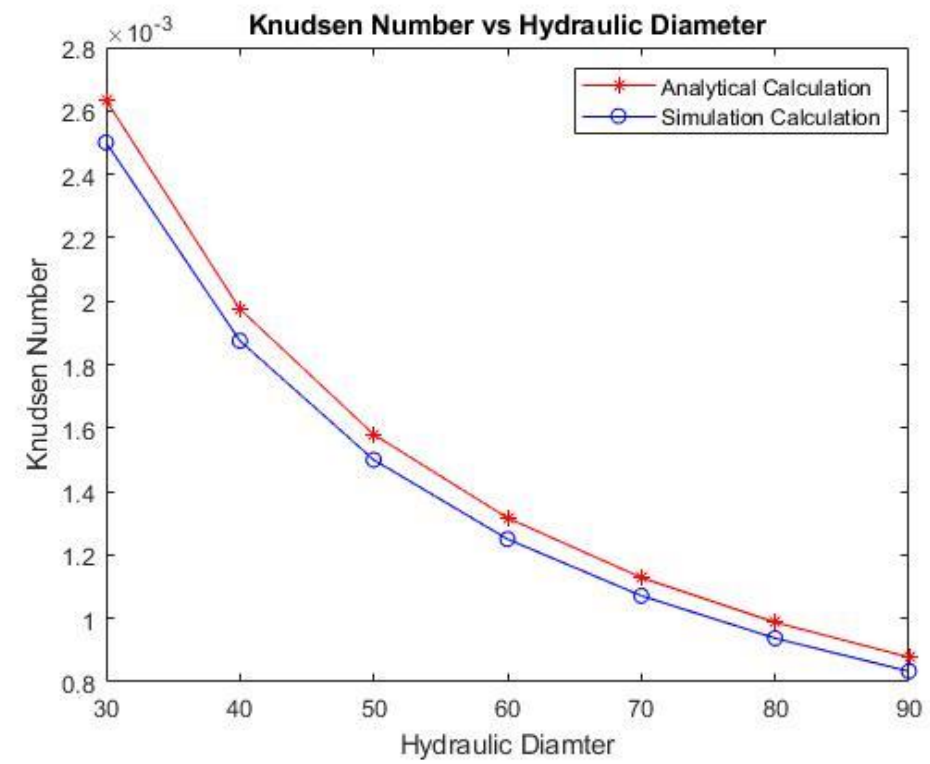

Figure 4.7 Comparing the Knudsen Number for $60 \mu \mathrm{m}$ Cylindrical Microchannel with COMSOL calculation and Analytical calculation

\subsection{Investigation of Velocity in The Microchannel}

The velocity of air in the silicon and pyrex microchannel of hydraulic diameter 60 $\mu \mathrm{m}$ is analysed with the wall thickness of $10 \mu \mathrm{m}, 20 \mu \mathrm{m}$ and $30 \mu \mathrm{m}$ for cylindrical and rectangular geometry. The impact in the velocity due to change in wall thickness is analysed to understand the influence of temperature $\left(70^{\circ} \mathrm{C}\right)$ (refer section 3.2) in the wall thickness. Table 4.4 and Figure 4.8 depict that the influence of wall thickness, material selection and geometry does not influence the velocity of air in the microchannel.

Silicon and pyrex material are chosen because of their thermal conductivity (refer to section 3.33) and simplifying the micromachining process. From Figure 4.8.c and 4.8.d the sniffing in time (which is the duration of the thermal transpiration effect) for the microchannel modelled using pyrex is about 300 seconds. The sniffing in time for the 
microchannel modelled using silicon is 7 seconds as shown in Figure 4.8.a and 4.8.b. Thus, from the analysis the thickness of the microchannel does not influence the velocity of air and the sniffing time for the cylindrical and rectangular microchannel modelled using pyrex is significantly longer than silicon. However, the material does not influence the velocity of the air. The velocity of air decreases with decrease in temperature difference from the outlet to the inlet of the microchannel.

Table 4.4 Maximum Velocity of air in the cylindrical and rectangular microchannel modelled using silicon and pyrex

\begin{tabular}{|l|l|l|l|l|}
\hline $\begin{array}{l}\text { Thickness of } \\
\text { the } \\
\text { microchannel }\end{array}$ & $\begin{array}{l}\text { Maximum Air } \\
\text { Velocity of Silicon } \\
\text { Cylindrical } \\
\text { Microchannel }\end{array}$ & $\begin{array}{l}\text { Maximum Air } \\
\text { Velocity of Silicon } \\
\text { Rectangular } \\
\text { Microchannel }\end{array}$ & $\begin{array}{l}\text { Maximum Air } \\
\text { Velocity of } \\
\text { Pyrex } \\
\text { Cylindrical } \\
\text { Microchannel }\end{array}$ & $\begin{array}{l}\text { Maximum } \\
\text { Air Velocity } \\
\text { of Pyrex } \\
\text { Rectangular } \\
\text { Microchannel }\end{array}$ \\
\hline $10 \mu \mathrm{m}$ & 0.32315 & 0.35435 & 0.29469 & 0.29732 \\
\hline $20 \mu \mathrm{m}$ & 0.2964 & 0.33113 & 0.29469 & 0.29372 \\
\hline $30 \mu \mathrm{m}$ & 0.30992 & 0.31547 & 0.28634 & 0.29322 \\
\hline
\end{tabular}

(a)

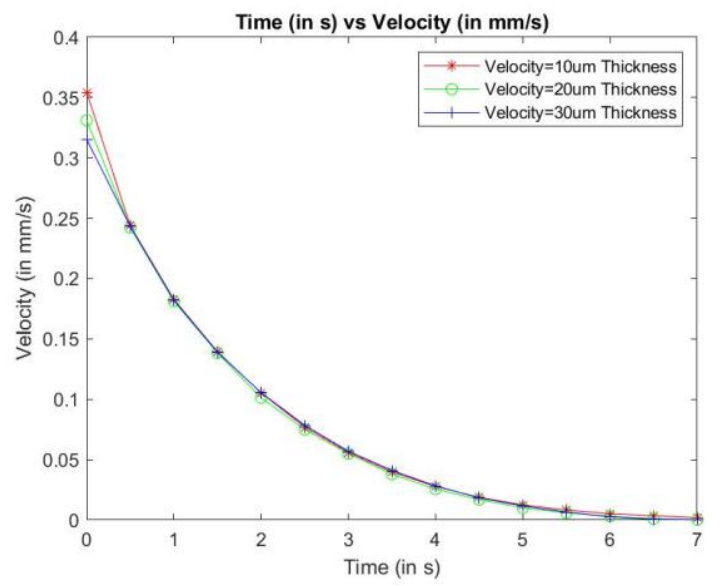

(b)

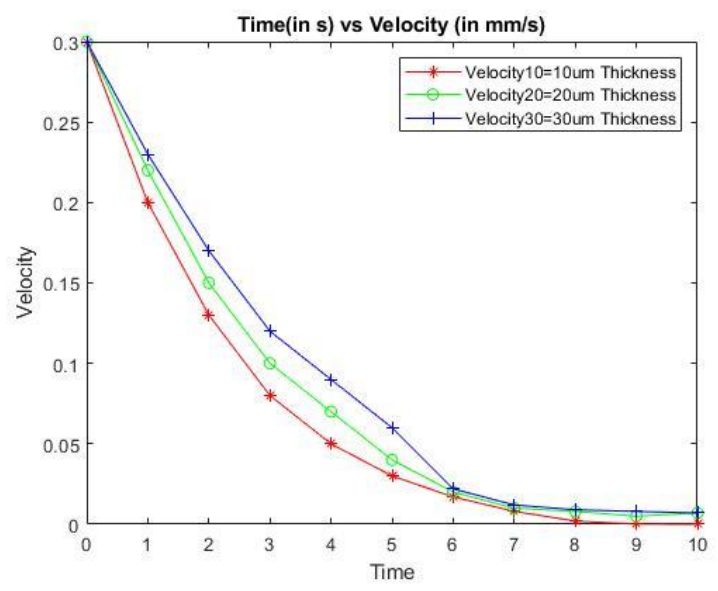


(c)

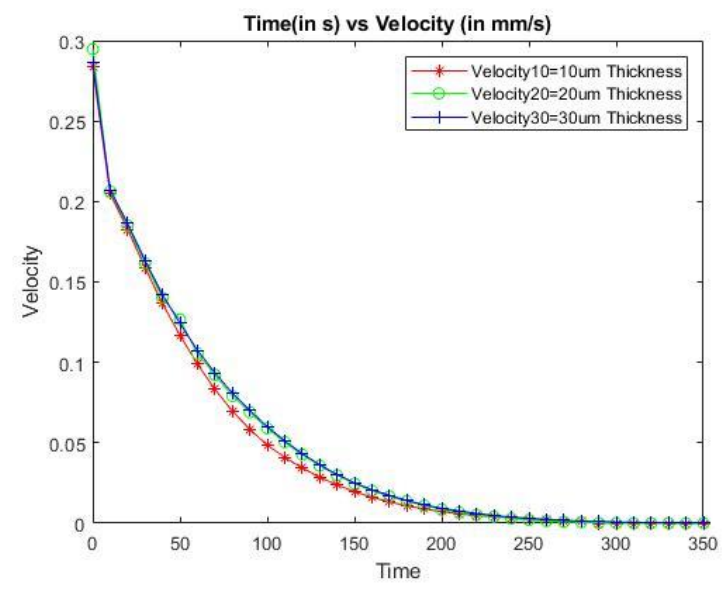

(d)

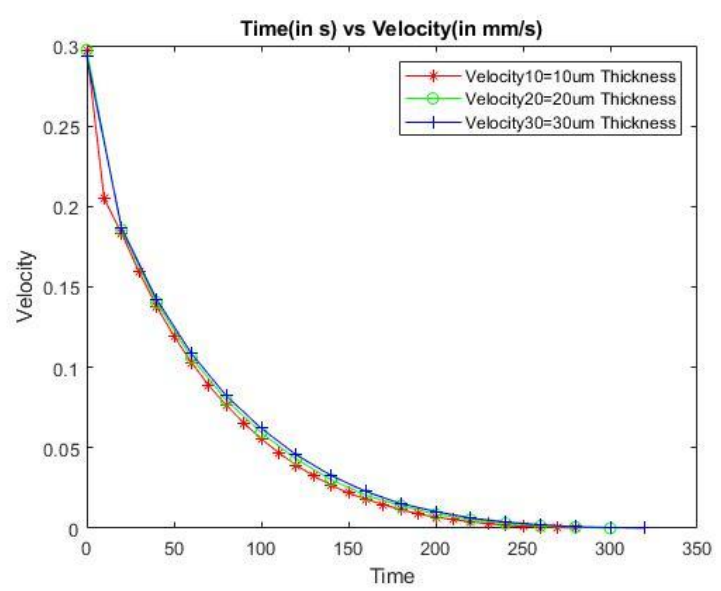

Figure 4.8 Comparing the velocity of a cylindrical and rectangular microchannel of hydraulic diameter 60 $\mu \mathrm{m}$ with varying thickness of $10 \mu \mathrm{m}, 20 \mu \mathrm{m}$ and $30 \mu \mathrm{m}$ at $70^{\circ} \mathrm{C}$ modelled using (a) silicon-cylindrical, (b) silicon-rectangular, (c) pyrex-cylindrical and (d) pyrex-rectangular.

\subsection{Investigation of Temperature Distribution in The Microchannel}

A maximum temperature of $70^{\circ} \mathrm{C}$ outlet of the cylindrical and rectangular microchannel modelled using silicon and pyrex of thickness $10 \mu \mathrm{m}, 20 \mu \mathrm{m}$, and $30 \mu \mathrm{m}$. The analysis is made to understand the ability to endure the microchannel's temperature difference for both cylindrical and rectangular geometries modelled using silicon and pyrex.

From Figure 4.9.a and 4.9.b, the time taken to reach the temperature of $70^{\circ} \mathrm{C}$ from the microchannel's outlet to inlet is given in Table 4.5 for cylindrical and rectangular geometries modelled using silicon. 
Table 4.5 Time taken to reach $70^{\circ} \mathrm{C}$ from the outlet to inlet for cylindrical and rectangular microchannel modelled using silicon material.

\begin{tabular}{|l|l|l|}
\hline $\begin{array}{l}\text { Thickness of the } \\
\text { microchannel }\end{array}$ & \multicolumn{2}{|l|}{ Microchannel modelled using silicon } \\
\cline { 2 - 3 } & Cylindrical & Rectangular \\
\hline $10 \mu \mathrm{m}$ & $9.5 \mathrm{~s}$ & $7.1 \mathrm{~s}$ \\
\hline $20 \mu \mathrm{m}$ & $10.4 \mathrm{~s}$ & $7.3 \mathrm{~s}$ \\
\hline $30 \mu \mathrm{m}$ & $11.6 \mathrm{~s}$ & $8.2 \mathrm{~s}$ \\
\hline
\end{tabular}

From Figure 4.9.c and 4.9.d, the time taken to reach the temperature of $70^{\circ} \mathrm{C}$ from the microchannel's outlet to inlet is given in Table 4.6 for cylindrical and rectangular geometries modelled using pyrex.

Table 4.6 Time taken to reach $70^{\circ} \mathrm{C}$ from the outlet to inlet for cylindrical and rectangular microchannel modelled using pyrex material.

\begin{tabular}{|l|l|l|}
\hline \multirow{2}{*}{$\begin{array}{l}\text { Thickness of the } \\
\text { microchannel }\end{array}$} & \multicolumn{2}{|l|}{ Microchannel modelled using pyrex } \\
\cline { 2 - 3 } & Cylindrical & Rectangular \\
\hline $10 \mu \mathrm{m}$ & $278 \mathrm{~s}$ & $280.9 \mathrm{~s}$ \\
\hline $20 \mu \mathrm{m}$ & $298.6 \mathrm{~s}$ & $300.1 \mathrm{~s}$ \\
\hline $30 \mu \mathrm{m}$ & $385.4 \mathrm{~s}$ & $317 \mathrm{~s}$ \\
\hline
\end{tabular}

Figure 4.9.a and 4.9.b depicts the temperature difference from the outlet to inlet of the cylindrical and rectangular microchannel modelled using silicon. Figure 4.9.c and 4.9.d depicts the temperature difference from the outlet to inlet of the cylindrical and rectangular microchannel modelled using pyrex. The temperature difference decreases with increasing time which shows that the heat's direction is from the outlet to the inlet. From the analysis, the microchannel's thickness and geometry do not have a significant influence in determining the heat flow in the microchannel. The temperature is given from the outlet to the volume of the microchannel. The time taken for the temperature to reach $70^{\circ} \mathrm{C}$ at the microchannel inlet is greater for pyrex than silicon. Hence, pyrex material is chosen as the candidate choice and rectangular geometry is selected to simplify the fabrication steps. 
(a)

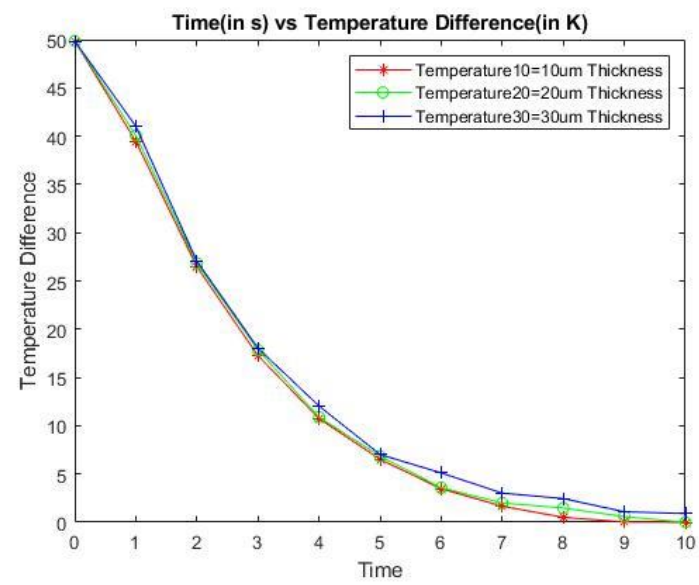

(c)

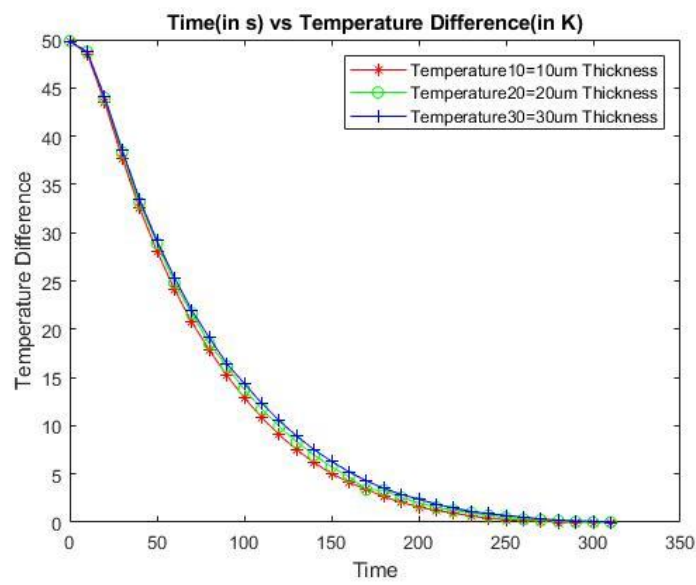

(b)

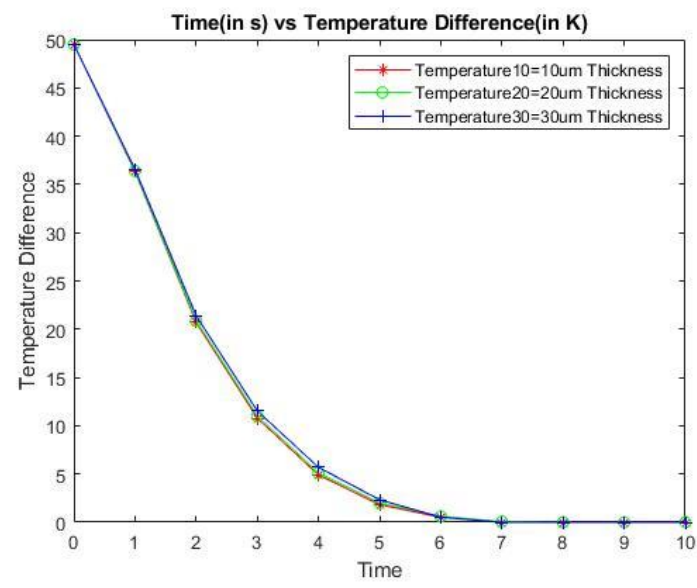

(d)

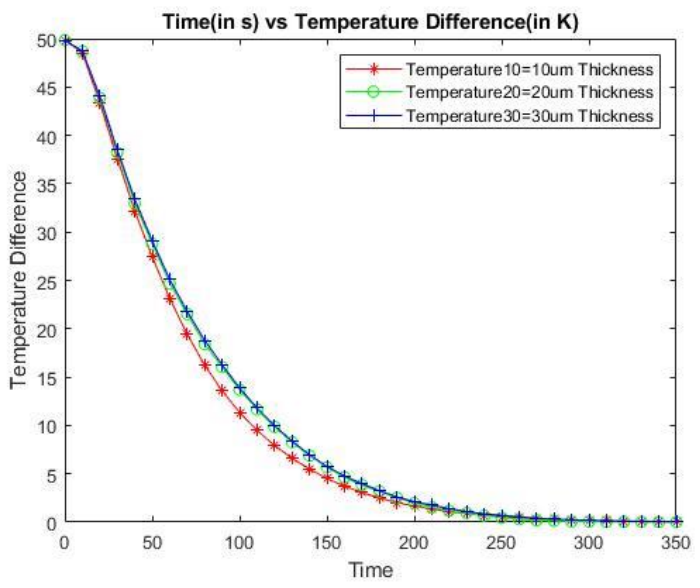

Figure 4.9 Comparing the temperature of a cylindrical and rectangular microchannel of hydraulic diameter $60 \mu \mathrm{m}$ with varying thickness of $10 \mu \mathrm{m}, 20 \mu \mathrm{m}$ and $30 \mu \mathrm{m}$ at $70^{\circ} \mathrm{C}$ modelled using (a) siliconcylindrical, (b) silicon-rectangular, (c) pyrex-cylindrical and (d) pyrex-rectangular

\subsection{Investigation of Back Flow Velocity of Pyrex Cylindrical and Rectangular}

\section{Microchannel}

When the microchannel's volume and surface reach $70^{\circ} \mathrm{C}$, the air is moved out from the outlet to the microchannel inlet, as shown in Figure 4.10.a and 410.b. The time taken for the air to move from the outlet to the inlet of the microchannel is called sniffing out time. In this section, the air velocity from the outlet to the inlet of the cylindrical and 
rectangular microchannel of thickness $10 \mu \mathrm{m}$ is modelled using pyrex material is analysed. The air velocity from the outlet of the microchannel to the inlet is called the backflow velocity.

After the threshold time of $280.9 \mathrm{~s}$ is reached, the air will be sniffed out from the microchannel because of zero temperature difference maintained from the outlet to the inlet of the microchannel. The pressure-driven force is triggered which causes the back flow. The backflow velocity commences when a temperature of $70^{\circ} \mathrm{C}$ is transferred from outlet to inlet, causing the entire microchannel to be heated to $70^{\circ} \mathrm{C}$ as shown in Figure 4.10 and 4.11.

The following analysis is made for the rectangular and cylindrical microchannel modelled using pyrex material because the sniffing time for the pyrex microchannel is $280.9 \mathrm{~s}$ which is higher than the silicon microchannel which has the sniffing time of $7.1 \mathrm{~s}$ as shown in Table 4.5 and 4.6. From Figure 4.12.a and 4.12.b the velocity of air being sniffed out of the microchannel is in the range of $10^{-4} \mathrm{~mm} / \mathrm{s}$ and from Figure 4.8 the air being sniffed inside the microchannel at time $t=0-1 \mathrm{~s}$ is in the range of 0.3 to $0.255 \mathrm{~mm} / \mathrm{s}$. From the analysis it is evident that the air velocity being sniffed inside the microchannel is greater than the air velocity sniffed out from the microchannel.

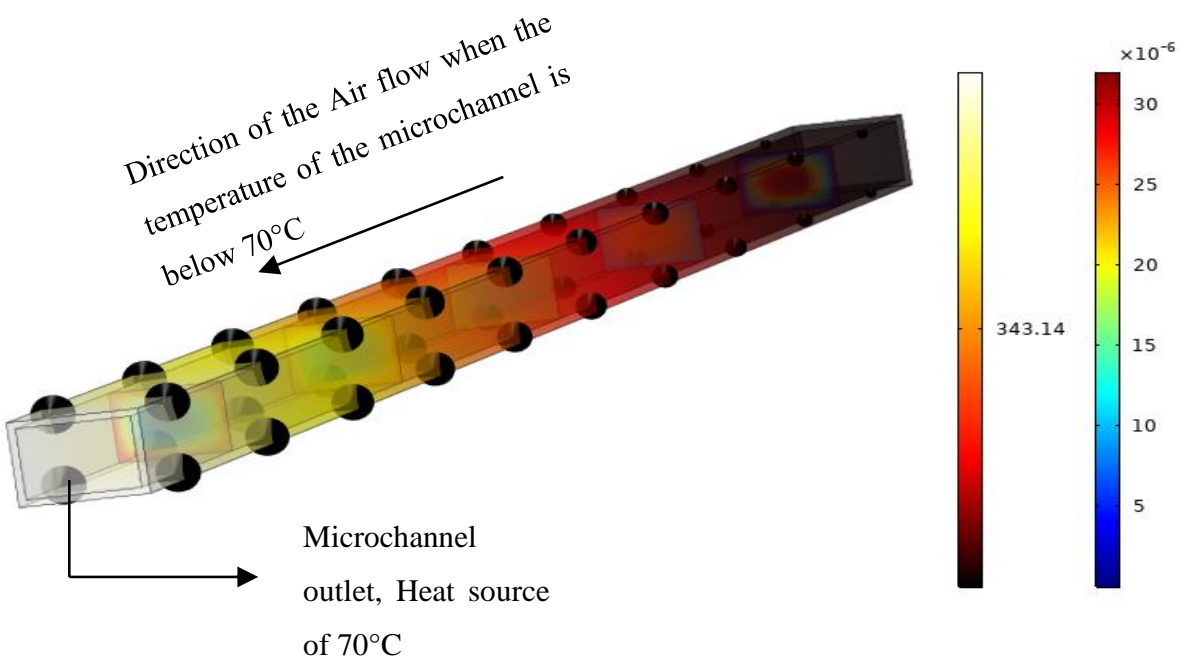

Figure 4.10 Air being sniffed into the microchannel at $69.99^{\circ} \mathrm{C}$ 


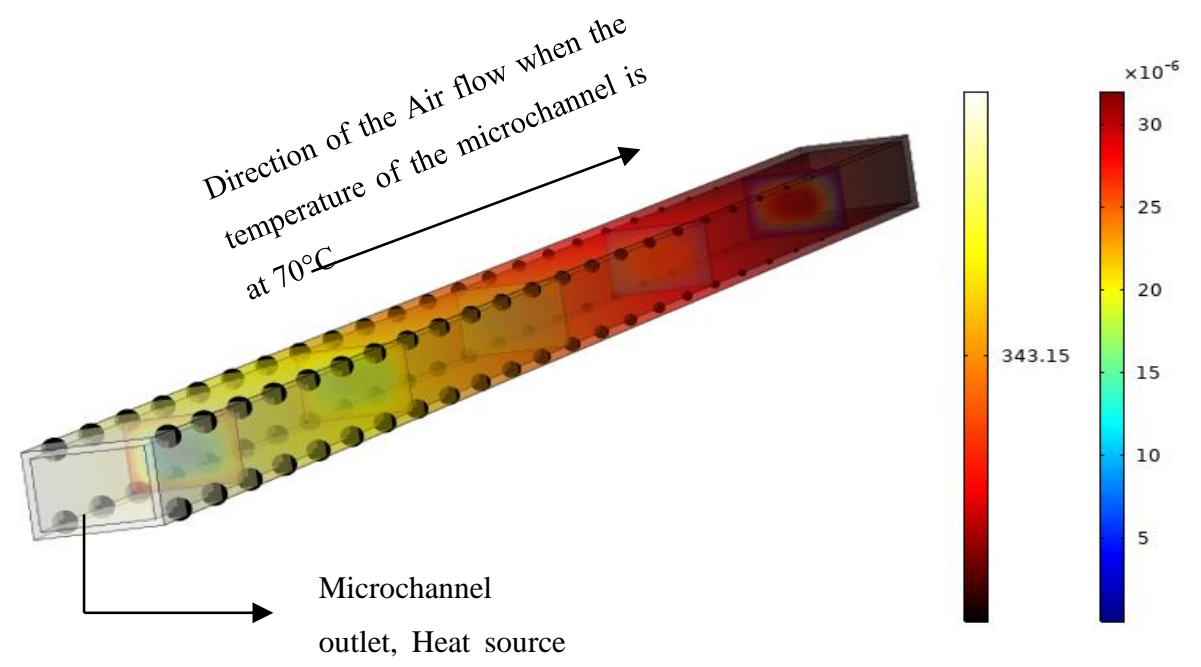

of $70^{\circ} \mathrm{C}$

Figure 4.11 Air being sniffed out from the microchannel at $70^{\circ} \mathrm{C}$

(a)

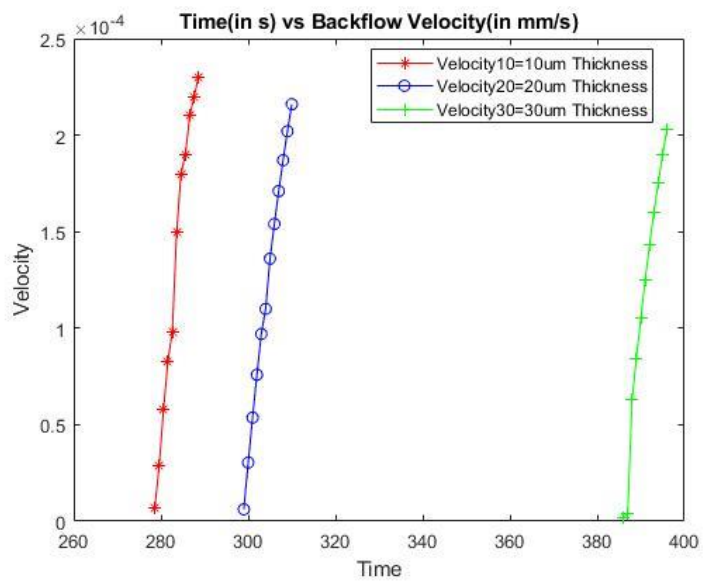

(b)

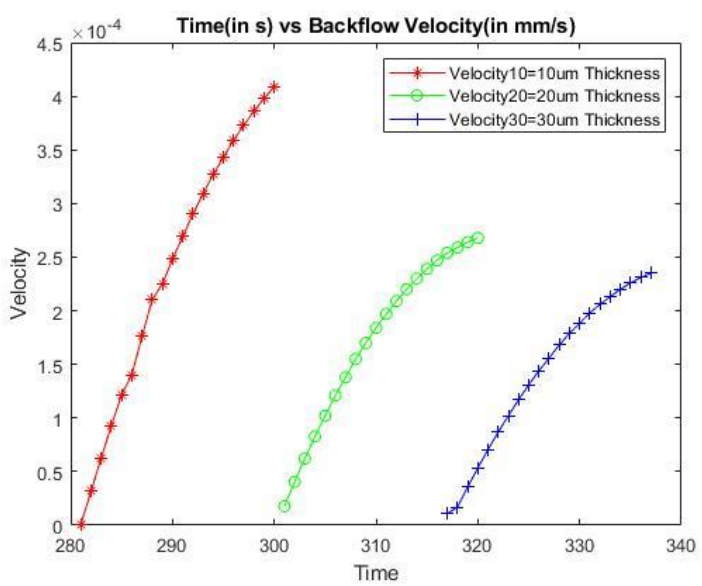

Figure 4.12 Comparing the backflow velocity of a cylindrical and rectangular microchannel of hydraulic diameter $60 \mu \mathrm{m}$ with varying thickness of $10 \mu \mathrm{m}, 20 \mu \mathrm{m}$ and $30 \mu \mathrm{m}$ at $70^{\circ} \mathrm{C}$ modelled using (a) pyrexcylindrical and (b) pyrex-rectangular 


\subsection{Velocity and Temperature Analysis of Pyrex Rectangular Microchannel for \\ Temperature of $40^{\circ} \mathrm{C}, 50^{\circ} \mathrm{C}$ and $60^{\circ} \mathrm{C}$}

The following analysis is made to understand the impact in the velocity by changing the heat source temperature at the outlet to $40^{\circ} \mathrm{C}, 50^{\circ} \mathrm{C}$ and $60^{\circ} \mathrm{C}$. From Figure 4.13.a, the time taken to reach temperature of $40^{\circ} \mathrm{C}$ and $50^{\circ} \mathrm{C}$ from the outlet to the inlet of the microchannel is greater than 500 second. The time taken to reach the temperature of $60^{\circ} \mathrm{C}$ is $304.5 \mathrm{~s}$. Thus, the sniffing time increases when the heat source temperature is reduced.

From Figure 4.13.b, the air velocity of the microchannel is $0.133 \mathrm{~mm} / \mathrm{s}, 0.172 \mathrm{~mm} / \mathrm{s}$ and $0.233 \mathrm{~mm} / \mathrm{s}$ for $40^{\circ} \mathrm{C}, 50^{\circ} \mathrm{C}$ and $60^{\circ} \mathrm{C}$. The air velocity at $70^{\circ} \mathrm{C}$ is $0.297 \mathrm{~mm} / \mathrm{s}$ from Table 4.4.

From the analysis the temperature and the air velocity are directly proportional. Thus, the temperature influences the air velocity. So, it is necessary to use the heater at the maximum temperature of $70^{\circ} \mathrm{C}$. The velocity increases with temperature and the time taken by the heat source to generate heat from the outlet to inlet increases with decrease in temperature. So, a volumetric study is accomplished to understand the influence of temperature in sniffing the volume of air into the microchannel.

(a)

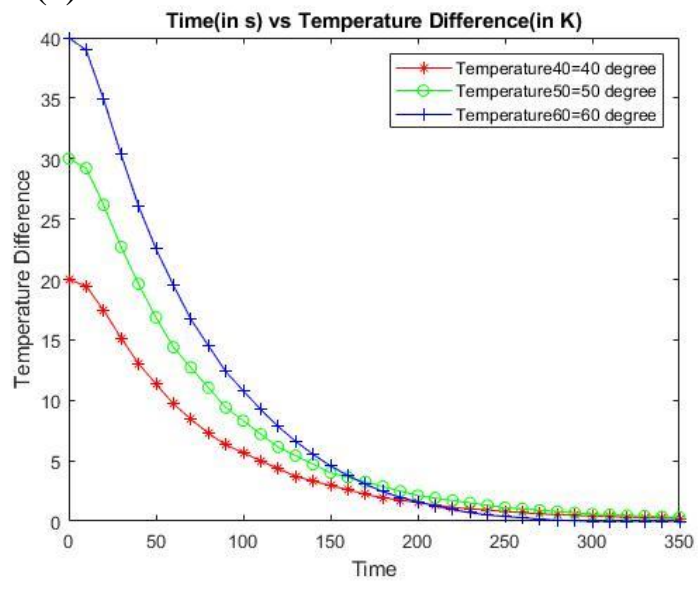

(b)

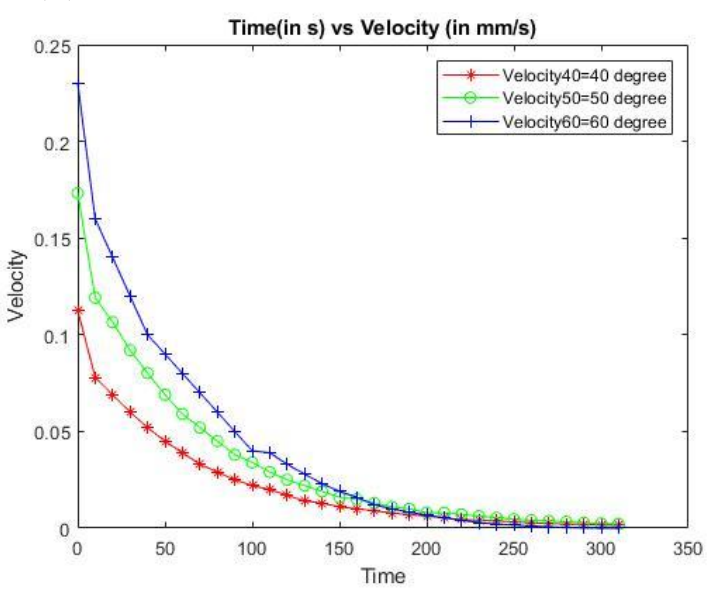

Figure 4.13 Comparing the (a) Temperature and (b) velocity of a rectangular microchannel of hydraulic diameter $60 \mu \mathrm{m}$ at $70^{\circ} \mathrm{C}$ modelled using pyrex 


\subsection{Volume of Air for Temperature of $40^{\circ} \mathrm{C}, 50^{\circ} \mathrm{C}, 60^{\circ} \mathrm{C}$ and $70^{\circ} \mathrm{C}$}

The volume of air being sniffed in is calculated analytically based on the results from the COMSOL simulation. The Navier-Stokes equation is used to solve the velocity of air during the sniffing in time. The width and breadth of the rectangular microchannel is $72 \mu \mathrm{m}$ and $52 \mu \mathrm{m}$, respectively which is used to calculate the velocity. The volume of air can be calculated by,

$$
\text { Volume of air in the microchannel }=[v * W * B * t]
$$

where $\mathrm{v}$ is the velocity of air, $\mathrm{W}$ is the width of the microchannel, $\mathrm{B}$ is the breadth of the microchannel and $\mathrm{t}$ is the sniffing time

From Figure 4.14, the volume of air sniffed in the microchannel increases with time. Even though the velocity of air decreases with time (refer section 4.6), the highest volume of air sniffed into the microchannel is $0.02 \mathrm{~mm}^{3}$ at a temperature of $70^{\circ} \mathrm{C}$. Hence, the quantity of the sample exposed to the sensor is higher irrespective of the velocity of the air. Table 4.7 shows that the volume of air sniffed inside the microchannel is more for $70^{\circ} \mathrm{C}$ than other temperature. Hence, the heat source has to be used in its maximum temperature.

Table 4.7 Volume of air sniffed into the microchannel for a temperature of $40^{\circ} \mathrm{C}, 50^{\circ} \mathrm{C}, 60^{\circ} \mathrm{C}$ and $70^{\circ} \mathrm{C}$

\begin{tabular}{|l|l|}
\hline $\begin{array}{l}\text { Temperature of the Heat } \\
\text { source }\end{array}$ & $\begin{array}{l}\text { Maximum Volume of } \\
\text { air sniffed }\end{array}$ \\
\hline $40^{\circ} \mathrm{C}$ & $0.0084 \mathrm{~mm}^{3}$ \\
\hline $50^{\circ} \mathrm{C}$ & $0.01302 \mathrm{~mm}^{3}$ \\
\hline $60^{\circ} \mathrm{C}$ & $0.017366 \mathrm{~mm}^{3}$ \\
\hline $70^{\circ} \mathrm{C}$ & $0.02196 \mathrm{~mm}^{3}$ \\
\hline
\end{tabular}




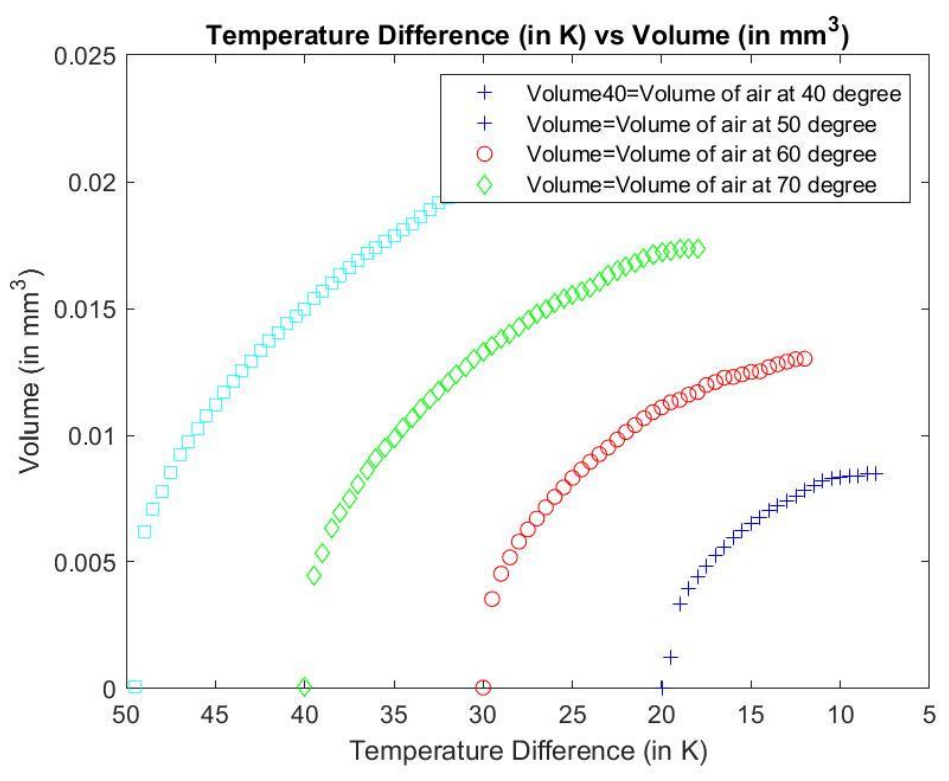

Figure 4.14 Comparing the volume of air sniffed in a rectangular microchannel of hydraulic diameter 60 um at $40^{\circ} \mathrm{C}, 50^{\circ} \mathrm{C}, 60^{\circ} \mathrm{C}$ and $70^{\circ} \mathrm{C}$ modelled using pyrex

\subsection{Convective Heat Transfer in Microchannel}

Heat transfer is a physical phenomenon of exchanging energy in a system from high temperature to low temperature. The heat transfer relies on a heat source that is the source of temperature and heat flow, which is the thermal energy direction. When the microchannel is heated, the heat energy is transferred from the microchannel outlet to the inlet as shown in Figure 4.4 and 4.5. The temperature from the heat source is assumed to be constant at $70^{\circ} \mathrm{C}$. Thus, at one point, the temperature from the outlet will reach the temperature of the inlet, which makes the entire microchannel to reach a temperature of $70^{\circ} \mathrm{C}$. The heat source is assumed to be turned off (refer section 3.2), and the microchannel cools down.

The heat energy is transferred from the microchannel which is at $70^{\circ} \mathrm{C}$ to the external environment which is assumed to be at room temperature $25^{\circ} \mathrm{C}$ through a process called convection. Newton's law of cooling states that the convection rate is directly proportional 
to the object's area being cooled, the difference in temperature of the surrounding fluid, and the object's surface temperature. It is given by the equation,

$$
\dot{Q}_{\text {conv }}=h A\left(T_{\infty}-T_{s}\right)
$$

where, $\dot{Q}_{\text {conv }}$ is the convective heat transfer rate, $\mathrm{h}$ is the heat transfer co-efficient, $\mathrm{A}$ is the surface area of the object, $T_{\infty}$ is the temperature of the external fluid and $T_{S}$ is the surface temperature of the object.

Convection is of two types which are

1) Natural convection

2) Force convection

\subsubsection{Natural Convection of the Rectangular Pyrex Microchannel}

Natural convection takes place due to difference in temperature in the microchannel and external environment. It occurs due to the natural circulation of air due to buoyancy and gravitational field. The heat transfer coefficient of air through natural convection is 2.5 to $25 \mathrm{~W} /\left(\mathrm{m}^{2} . \mathrm{K}\right)[127]$.

\subsection{Investigation of Cool-Down Time Through Natural Convection}

The rectangular microchannel's surface temperature is set to $70^{\circ} \mathrm{C}$ to find the cooldown time of the microchannel. COMSOL provides the heat transfer coefficient of air. Figure 4.15 depicts that the time taken by the microchannel to reach room temperature of $25^{\circ} \mathrm{C}$ is approximately 5 seconds. Further analysis is made by giving the heat transfer coefficient from 2.5 to $25 \mathrm{~W} /\left(\mathrm{m}^{2} . \mathrm{K}\right)$ shown in Table 4.8 .

Table 4.8 Cool-down time of the microchannel for different heat transfer co-efficient

\begin{tabular}{|l|l|}
\hline Heat transfer co-efficient & Cooling time of the microchannel \\
\hline $2.5 \mathrm{~W} /\left(\mathrm{m}^{2} . \mathrm{K}\right)$ & $19 \mathrm{~s}$ \\
\hline $10 \mathrm{~W} /\left(\mathrm{m}^{2} \cdot \mathrm{K}\right)$ & $4 \mathrm{~s}$ \\
\hline $25 \mathrm{~W} /\left(\mathrm{m}^{2} \cdot \mathrm{K}\right)$ & $3 \mathrm{~s}$ \\
\hline
\end{tabular}


From the analysis, the heat transfer coefficient is inversely proportional to the rate of cooling - the heat transfer coefficient increases as the time taken to cool down the microchannel decreases. Thus, the cool-down time of the microchannel depends on the external factors such as velocity of the air, temperature of the air.

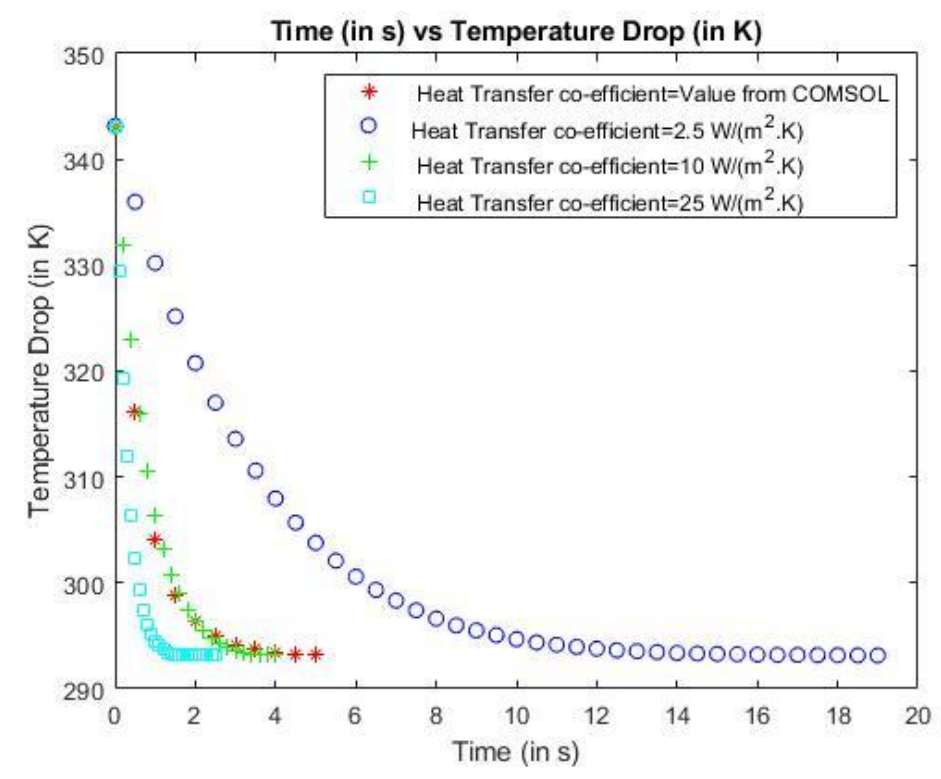

Figure 4.15 Comparing the cool-down time of a rectangular microchannel of hydraulic diameter $60 \mu \mathrm{m}$ at $70^{\circ} \mathrm{C}$ modelled using pyrex material for the heat transfer coefficient of $2.5 \mathrm{~W} /\left(\mathrm{m}^{2} . \mathrm{K}\right), 10 \mathrm{~W} /\left(\mathrm{m}^{2} . \mathrm{K}\right), 25 \mathrm{~W} /$

$$
\left.\left(m^{2} \cdot K\right)\right)
$$

\subsection{Conclusion and Summary}

In this chapter the physics and boundary conditions behind the finite element analysis is presented. Since analytical modelling cannot be applied to study the velocity by coupling the temperature in the entire domain of the microchannel, finite element analysis has been employed. The swept meshing is used because unlike tetrahedral and triangular meshes, swept mesh creates less elements that accounts for the distorted domains in the geometry. During the analysis it is observed that the temperature influences the velocity and volume of air pumped inside the microchannel. The geometry and thickness of the microchannel does not have major impacts on the velocity and temperature of the microchannel. Hence, a rectangular microchannel design was chosen to simplify the microfabrication step. Natural convection method is applied to calculate the cooling down 
time for the microchannel, which is in the range of $2.5 \mathrm{~W} /\left(\mathrm{m}^{2} . \mathrm{K}\right)$ to $25 \mathrm{~W} /\left(\mathrm{m}^{2} . \mathrm{K}\right)$ when exposed to a real-time environment. The microchannel cooling rate decreases with increase in the heat transfer co-efficient. 


\section{CHAPTER 5}

CONCLUSION AND FUTURE WORK

The odors of plants are complex mixture of chemically heterogenous VOCs. More than 30000 different compounds emitted by plants in physiological conditions. Plants can synthesize and store the VOCs. Plants have a broad range of defense mechanisms for combatting infections, attacks by herbivorous insects, and mechanical damage. One of these protection strategies involves emitting specific VOCs to fight potential attackers. These defenses are often divided into direct defenses and indirect defenses. In direct defenses, plants emit repellent VOCs to reduce insect attacks, while, in indirect defenses, VOCs can attract predators to battle pests. It is clear that VOCs play significant roles in plant communication and present promising functionality for improving crop protection. The VOCs provide functional information about the growth rate of the plants and the status of their health. Thus, enhancing the possibilities of using non-invasive detection to monitor the plant status. Compared with traditional gas chromatography-mass spectrometry (GCMS) techniques, electronic noses are non-invasive and can be a rapid, cost-effective option for several applications. E-nose comprises of an array of sensor and pattern recognition algorithm to detect the VOCs.

The external pumping mechanism is used to collect the samples from the plant to deliver them to the array of sensors in the E-nose. The main drawback of the external pumping mechanism is that it has movable parts which are prone to damage on exposing to the external environment. The movable parts in a mechanical micropump which works on actuating principle are complex in fabrication and requires high maintenance. In order to overcome the drawback non-mechanical pumping mechanism which involves collecting the sample through a microchannel which is induced by temperature gradients is one of the ideal solutions. This has the advantages of having no moving parts, simple structure, and less power consumption.

This research focuses on the gas flow induced by the thermal transpiration effect. The critical parameters of the cylindrical and rectangular microchannels are investigated and modelled using silicon and pyrex material because of its simplifying fabrication procedure. The maximum temperature generated from the heat source is assumed to be 
$70^{\circ} \mathrm{C}$. Hence, the microchannel's hydraulic diameter should be designed such that it falls within the slip flow regime of $0.001<\mathrm{Kn}<0.1$. Due to the temperature source at the outlet of the microchannel, the air becomes rarefied, and it starts to creep towards the heat source from the inlet. This is called thermal transpiration effect and it is the basic principle of operation of the device. Furthermore, the Navier-stokes equations is used to analyse the air velocity that creeps towards the temperature source at the outlet. The temperature applied at the outlet is analysed using the energy equation. The thermal gradients at the walls of the microchannel causes a formation of a thin layer adjacent to the microchannel's walls where the velocity is analysed by using maxwell's slip boundary and Smoluchowski temperature jump conditions.

\subsection{Conclusion and Summary}

The E-nose system comprises of an array of sensors and the pattern recognition system connected with an external pumping mechanism. The thermal transpiration effect is used as a pumping mechanism to pump the air into the microchannel and deliver it to the sensor array. The microchannels are used in several disciplined of engineering such as a heat sink and heat exchangers in mechanical and automotive industries and lab-on-chip in chemical industries. Based on the requirements and applications, the geometries of the microchannel are designed

The performance of the E-nose system depends on the temperature gradient generated from the microheater which delivers air sample to the sensor array. The air velocity depends on the air density, impurities in the air and humidity in the air. Hence, these factors can be considered only after fabrication of the microchannel. These factors are verified experimentally. In this work, the microchannel is implemented as a sniffer. So, the rectangular and cylindrical geometry are considered which are the widely used designs for gas transportation.

By using the mean free path values of the gas molecules and the hydraulic diameter of the microchannel, the $\mathrm{Kn}$ is calculated. The analytical calculated values of the $\mathrm{Kn}$ are compared with the values from COMSOL simulation. Thus, critical design parameters are analysed by comparing the Kn values in the slip flow regime. The velocity profile in the microchannel is analysed by varying the wall thickness of the microchannel to understand 
the relationship between the velocity and wall thickness. The simulation results depict that air velocity in the microchannel is independent of the wall thickness. The relation between temperature and is analysed by changing the wall thickness of the cylindrical and rectangular microchannel. From the simulation results, the wall thickness is independent of the applied temperature. The relationship between the temperature and velocity revealed that the velocity does not increase drastically however, the volume of air which is sniffed into the microchannel increases with temperature. The investigation in the backflow velocity or reverse thermal transpiration effect revealed that the velocity of air which is sniffed out from the microchannel is less than the backflow velocity. When the microchannel is heated to the maximum temperature the time taken by the microchannel to cool-down in natural convection is inversely proportional to the heat transfer coefficient. The rate of cooling depends on the temperature of air in the external environment.

\subsection{Future Work}

The major problem with the external pumping mechanism that involves moving parts is overcome by the principle of thermal transpiration effect in a microchannel. The analysis of the material such as silicon and pyrex revealed that the material with low thermal conductivity has better control in temperature and air velocity. Hence, material like Polyether ether ketone (PEEK) and Polydimethylsiloxane (PDMS) which has the thermal conductivity of $0.25 \mathrm{~W} / \mathrm{m} . \mathrm{K}$ and $0.56 \mathrm{~W} / \mathrm{m} . \mathrm{K}$ can be implemented to analyse the air velocity and temperature distribution in the microchannel as these materials have simplifying fabrication procedures. A detailed fabrication procedure should be implemented to design the proposed microchannel. The TMAC value should be further investigated for PEEK and PDMS. 


\section{BIBLIOGRAPHY}

[1] 'Greenhouse vegetable production in Canada'. https://www.hortcouncil.ca/en/about-us/horticulture-stats/greenhouse-vegetableproduction-in-canada/ (accessed Mar. 21, 2021).

[2] 'Statistical Overview of the Canadian Greenhouse Vegetable Industry, 2019'. https://www.agr.gc.ca/eng/canadas-agriculture-sectors/horticulture/horticulture-sectorreports/statistical-overview-of-the-canadian-greenhouse-vegetable-industry2019/?id=1609252451699\#a1.1 (accessed Mar. 21, 2021).

[3] 'Canada's booming greenhouse industry on the lookout for innovative growing solutions'. $\quad$ https://www.s-ge.com/en/article/global-opportunities/20191-c6-canadagreenhouse-industry (accessed Mar. 21, 2021).

[4] A. V. F. Ngowi, T. J. Mbise, A. S. M. Ijani, L. London, and O. C. Ajayi, 'Smallholder Vegetable Farmers in Northern Tanzania: Pesticides Use Practices, Perceptions, Cost and Health Effects', Crop Prot., vol. 26, no. 11, pp. 1617-1624, 2007, doi: 10.1016/j.cropro.2007.01.008.

[5] R. Musebe, A. Massawe, T. Mansuet, M. Kimani, U. Kuhlmann, and S. Toepfer, 'Achieving Rational Pesticide Use in Outdoor Tomato Production Through Farmer Training and Implementation of a Technical Guideline', J. Agric. Ext. Rural Dev., vol. 6, no. 12 , pp. 367-381, 2014, doi: 10.5897/JAERD14.0569.

[6] J. H. Syed et al., 'Pesticide Residues in Fruits and Vegetables from Pakistan: A Review of the Occurrence and Associated Human Health Risks', Environ. Sci. Pollut. Res., vol. 21, no. 23, pp. 13367-13393, 2014, doi: 10.1007/s11356-014-3117-z. 
[7] V. Kumar, J. Dhar, and H. S. Bhatti, 'Bifurcation Dynamics of a Plant-Pest-Natural Enemy System in Polluted Environment Incorporating Gestation Delays', Ric. di Mat., vol. 69, no. 2, pp. 533-551, 2020, doi: 10.1007/s11587-019-00455-х.

[8] S. Toepfer et al., 'Sustainable Pest Management Through Improved Advice in Agricultural Extension', Sustain., vol. 12, no. 17, 2020, doi: 10.3390/SU12176767.

[9] M. Qaim, 'Role of New Plant Breeding Technologies for Food Security and Sustainable Agricultural Development', Appl. Econ. Perspect. Policy, vol. 42, no. 2, pp. 129-150, 2020, doi: 10.1002/aepp.13044.

[10] M. P. López-Gresa et al., 'A New Role for Green Leaf Volatile Esters in Tomato Stomatal Defense Against Pseudomonas Syringe pv. Tomato', Front. Plant Sci., vol. 871, no. December, pp. 1-12, 2018, doi: 10.3389/fpls.2018.01855.

[11] J. Laothawornkitkul et al., 'Discrimination of Plant Volatile Signatures by an Electronic Nose: A Potential Technology for Plant Pest and Disease Monitoring', Environ. Sci. Technol., vol. 42, no. 22, pp. 8433-8439, 2008, doi: 10.1021/es801738s.

[12] K. Bürling, M. Hunsche, and G. Noga, 'Use of Blue-Green and Chlorophyll Fluorescence Measurements for Differentiation Between Nitrogen Deficiency and Pathogen Infection in Winter Wheat', J. Plant Physiol., vol. 168, no. 14, pp. 1641-1648, 2011, doi: 10.1016/j.jplph.2011.03.016.

[13] A. K. Mahlein, E. C. Oerke, U. Steiner, and H. W. Dehne, 'Recent Advances in Sensing Plant Diseases for Precision Crop Protection', Eur. J. Plant Pathol., vol. 133, no. 1, pp. 197-209, 2012, doi: 10.1007/s10658-011-9878-z.

[14] X. Xu, F. Tian, S. X. Yang, Q. Li, J. Yan, and J. Ma, 'A solid trap and Thermal Desorption System with Application to a Medical Electronic Nose', Sensors, vol. 8, no. 11, pp. 6885-6898, 2008, doi: 10.3390/s8116885.

[15] S. Cui, J. Wu, J. Wang, and X. Wang, 'Discrimination of American Ginseng and Asian Ginseng Using Electronic nose and Gas Chromatography-Mass Spectrometry Coupled with Chemometrics', J. Ginseng Res., vol. 41, no. 1, pp. 85-95, 2017, doi: 
10.1016/j.jgr.2016.01.002.

[16] R. Capuano et al., 'Simultaneous Proton Transfer Reaction-Mass Spectrometry and Electronic Nose Study of the Volatile Compounds Released by Plasmodium Falciparum Infected Red Blood Cells in Vitro', Sci. Rep., vol. 9, no. 1, pp. 1-10, 2019, doi: 10.1038/s41598-019-48732-x.

[17] A. A. Vasiliev et al., 'Non-silicon MEMS Platforms for Gas Sensors', Sensors Actuators, B Chem., vol. 224, pp. 700-713, 2016, doi: 10.1016/j.snb.2015.10.066.

[18] Y. Qin, S. An, and Y. B. Gianchandani, 'Arrayed Architectures for Multi-stage SiMicromachined High-Flow Knudsen Pumps', J. Micromechanics Microengineering, vol. 25, no. 11, 2015, doi: 10.1088/0960-1317/25/11/115026.

[19] S. An, N. K. Gupta, and Y. B. Gianchandani, 'A Si-Micromachined 162-Stage Two-Part Knudsen Pump for On-Chip Vacuum', J. Microelectromechanical Syst., vol. 23, no. 2, pp. 406-416, 2014, doi: 10.1109/JMEMS.2013.2281316.

[20] D. J. Laser and J. G. Santiago, 'A Review of Micropumps', J. Micromechanics Microengineering, vol. 14, no. 6, 2004, doi: 10.1088/0960-1317/14/6/R01.

[21] J. W. Gardner and P. N. Bartlett, 'A Brief History of Electronic Noses', Sensors Actuators B. Chem., vol. 18, no. 1-3, pp. 210-211, 1994, doi: 10.1016/09254005(94)87085-3.

[22] F. E. Schaller, Emmanuelle, Jacques O. Bosset, "Electronic Noses" and Their Application to Food', LWT - Food Sci. Technol., vol. 31, no. 4, pp. 305-316, 1998.

[23] D. James, S. M. Scott, Z. Ali, and W. T. O'Hare, 'Chemical Sensors for Electronic Nose Systems', Microchim. Acta, vol. 149, no. 1-2, pp. 1-17, 2005, doi: 10.1007/s00604004-0291-6.

[24] A. D. Wilson and M. Baietto, 'Applications and Advances in Electronic-Nose Technologies', Sensors, vol. 9, no. 7, pp. 5099-5148, 2009, doi: 10.3390/s90705099.

[25] D. B. Tuckerman and R. F. W. Pease, 'High-Performance Heat Sinking for VLSI', 
IEEE Electron Device Lett., vol. EDL-2, no. 5, pp. 126-129, 1981, doi: 10.1109/EDL.1981.25367.

[26] S. G. Kandlikar, 'Fundamental Issues Related to Flow Boiling in Minichannels and Microchannels', Exp. Therm. Fluid Sci., vol. 26, no. 2-4, pp. 389-407, 2002, doi: 10.1016/S0894-1777(02)00150-4.

[27] W. Qu and I. Mudawar, 'Experimental and Numerical Study of Pressure Drop and Heat Transfer in a Single-Phase Micro-Channel Heat Sink', Int. J. Heat Mass Transf., vol. 45, no. 12, pp. 2549-2565, 2002, doi: 10.1016/S0017-9310(01)00337-4.

[28] W. Qu and I. Mudawar, 'Flow Boiling Heat Transfer in Two-Phase Micro-Channel Heat Sinks-I. Experimental Investigation and Assessment of Correlation Methods', Int. J. Heat Mass Transf., vol. 46, no. 15, pp. 2755-2771, 2003, doi: 10.1016/S00179310(03)00041-3.

[29] M. J. Jackson and W. Ahmed, Micro and Nanomanufacturing, vol. 2, no. January. 2017.

[30] P. Taylor and W. J. Grande, 'Evolution of Microchannel Flow Passages-Thermohydraulic Performance and Fabrication Technology Evolution of Microchannel Flow Passages — Thermohydraulic Performance and', Mech. Eng., no. February 2012, pp. 37-41, 2010, doi: 10.1080/01457630390116077.

[31] N. D. Stevanovic, 'A New Analytical Solution of Microchannel Gas Flow', J. Micromechanics Microengineering, vol. 17, no. 8, pp. 1695-1702, 2007, doi: 10.1088/0960-1317/17/8/036.

[32] S. Chandra and O. Prakash, 'Heat Transfer in Microchannel Heat Sink: Review', Int. Conf. Recent Adv. Mech. Eng., no. October 2016, 2016, [Online]. Available: https://www.sciencedirect.com/science/article/pii/S0735193300001329.

[33] Y. Han, Y. Liu, M. Li, and J. Huang, 'A Review of Development of Micro-Channel Heat Exchanger Applied in Air-Conditioning System', Energy Procedia, vol. 14, pp. 148153, 2012, doi: 10.1016/j.egypro.2011.12.910. 
[34] S. H. Lee, M. Park, C. G. Park, J. E. Lee, M. R. Prausnitz, and Y. Bin Choy, 'Microchip for Sustained Drug Delivery by Diffusion Through Microchannels', AAPS PharmSciTech, vol. 13, no. 1, pp. 211-217, 2012, doi: 10.1208/s12249-011-9743-6.

[35] F. A. Shaikh and V. M. Ugaz, 'Collection, focusing, and metering of DNA in microchannels using addressable electrode arrays for portable low-power bioanalysis', Proc. Natl. Acad. Sci. U. S. A., vol. 103, no. 13, pp. 4825-4830, 2006, doi: 10.1073/pnas.0506848103.

[36] M. Sneha Maria, P. E. Rakesh, T. S. Chandra, and A. K. Sen, 'Capillary Flow of Blood in a Microchannel with Differential Wetting for Blood Plasma Separation and OnChip Glucose Detection', Biomicrofluidics, vol. 10, no. 5, 2016, doi: 10.1063/1.4962874.

[37] Q. Mei, R. Khnouf, A. Simon, and Z. H. Fan, 'Protein Synthesis in a Device with Nanoporous Membranes and Microchannels', Lab Chip, vol. 10, no. 19, pp. 2541-2545, 2010, doi: 10.1039/c005233g.

[38] A. R. Bahadorimehr, Y. Jumril, and B. Y. Majlis, 'Low Cost Fabrication of Microfluidic Microchannels for Lab-On-a-Chip Applications', 2010 Int. Conf. Electron. Devices, Syst. Appl. ICEDSA 2010 - Proc., pp. 242-244, 2010, doi: 10.1109/ICEDSA.2010.5503067.

[39] S. J. Hymel, H. Lan, H. Fujioka, and D. B. Khismatullin, 'Cell Trapping in YJunction Microchannels: A Numerical Study of the Bifurcation Angle Effect in Inertial Microfluidics', Phys. Fluids, vol. 31, no. 8, 2019, doi: 10.1063/1.5113516.

[40] S. Damiati, U. B. Kompella, S. A. Damiati, and R. Kodzius, 'Microfluidic Devices for Drug Delivery Systems and Drug Screening', Genes (Basel)., vol. 9, no. 2, 2018, doi: 10.3390/genes9020103.

[41] H. Transfer, 'Rarefaction Effect on Heat Transfer and Fluid Flow in Microchannel', 2nd Int. Conf. Energy Convers. Conserv., pp. 1-5, 2008.

[42] G. L. Morini, M. Lorenzini, and M. Spiga, 'A Criterion For The Exprimental Validation of The Slip-Flow Models For Incompressible Rarefied Gases Through 
Microchannel', Microfluid Nanofluid, vol. 1, pp. 190-196, 2005, doi: https://doi.org/10.1007/s10404-004-0028-1.

[43] A. Agrawal, 'A Comprehensive Review on Gas Flow in Microchannels', Int. J. Micro-Nano Scale Transp., vol. 2, no. 1, pp. 1-40, 2011, doi: 10.1260/1759-3093.2.1.1.

[44] G. Cai, L. Xue, H. Zhang, and J. Lin, 'A review on Micromixers', Micromachines, vol. 8, no. 9, 2017, doi: 10.3390/mi8090274.

[45] A. Banerjee, A. K. Nayak, and B. Weigand, 'Enhanced Mixing and Flow Reversal in a Modulated Microchannel', Int. J. Mech. Sci., vol. 155, no. February, pp. 430-439, 2019, doi: 10.1016/j.ijmecsci.2019.02.023.

[46] D. Nouri, A. Zabihi-Hesari, and M. Passandideh-Fard, 'Rapid Mixing in Micromixers Using Magnetic Field', Sensors Actuators, A Phys., vol. 255, pp. 79-86, 2017, doi: 10.1016/j.sna.2017.01.005.

[47] S. Y. K. Lee, M. Wong, and Y. Zohar, 'Gas Flow in Microchannels with Bends', J. Micromechanics Microengineering, vol. 11, no. 6, pp. 635-644, 2001, doi: 10.1088/0960$1317 / 11 / 6 / 304$.

[48] S. Maharudrayya, S. Jayanti, and A. P. Deshpande, 'Pressure Losses in Laminar Flow Through Serpentine Channels in Fuel Cell Stacks', J. Power Sources, vol. 138, no. 1-2, pp. 1-13, 2004, doi: 10.1016/j.jpowsour.2004.06.025.

[49] M. Shams, M. Shojaeian, C. Aghanajafi, and S. A. R. Dibaji, 'Numerical Simulation of Slip Flow Through Rhombus Microchannels', Int. Commun. Heat Mass Transf., vol. 36, no. 10, pp. 1075-1081, 2009, doi: 10.1016/j.icheatmasstransfer.2009.07.010.

[50] A. A. Alfaryjat, H. A. Mohammed, N. M. Adam, M. K. A. Ariffin, and M. I. Najafabadi, 'Influence of Geometrical Parameters of Hexagonal, Circular, and Rhombus Microchannel Heat Sinks on the Thermohydraulic Characteristics', Int. Commun. Heat Mass Transf., vol. 52, pp. 121-131, 2014, doi: 10.1016/j.icheatmasstransfer.2014.01.015.

[51] I. Graur and F. Sharipov, 'Gas Flow Through an Elliptical Tube Over The Whole 
Range of The Gas Rarefaction', Eur. J. Mech. B/Fluids, vol. 27, no. 3, pp. 335-345, 2008, doi: 10.1016/j.euromechflu.2007.07.003.

[52] J. Pitakarnnop et al., 'Slip Flow in Triangular and Trapezoidal Microchannels', Int. J. Heat Technol., vol. 26, 2007.

[53] S. Giannoukos, B. Brkić, S. Taylor, A. Marshall, and G. F. Verbeck, 'Chemical Sniffing Instrumentation for Security Applications', Chem. Rev., vol. 116, no. 14, pp. 8146-8172, 2016, doi: 10.1021/acs.chemrev.6b00065.

[54] G. S. Settles, 'Sniffers: Fluid-dynamic sampling for olfactory trace detection in nature and homeland security - The 2004 freeman scholar lecture', J. Fluids Eng. Trans. ASME, vol. 127, no. 2, pp. 189-218, 2005, doi: 10.1115/1.1891146.

[55] A. M. Andrew et al., 'Probabilistic Neural Network Based Olfactory Classification for Household Burning in Early Fire Detection Application', 2013 IEEE Conf. Open Syst. ICOS 2013, pp. 221-225, 2013, doi: 10.1109/ICOS.2013.6735078.

[56] H. Maricou, D. Pereira, L. Verschuere, S. Philips, and W. Verstraete, 'Measurements of Some Volatile Compounds by Means of The Electronic Nose', Water. Air. Soil Pollut., vol. 107, no. 1-4, pp. 423-442, 1998, doi: 10.1023/A:1019882724915.

[57] V. Martini-Laithier, I. Graur, S. Bernardini, K. Aguir, P. Perrier, and M. Bendahan, 'Ammonia Detection by a Novel Pyrex Microsystem Based on Thermal Creep Phenomenon', Sensors Actuators, B Chem., vol. 192, pp. 714-719, 2014, doi: 10.1016/j.snb.2013.10.120.

[58] A. D. Wilson, D. G. Lester, and C. S. Oberle, 'Development of Conductive Polymer Analysis for The Rapid Detection and Identification of Phytopathogenic Microbes', Phytopathology, vol. 94, no. 5, pp. 419-431, 2004, doi: 10.1094/PHYTO.2004.94.5.419.

[59] O.Reynolds, 'On Certain Dimensional Properties of Matter in the Gaseous State', R. Soc., vol. 308, no. 795, pp. 379-430, 1878, doi: 10.1098/rstb.1983.0080.

[60] N. K. Gupta, S. An, and Y. B. Gianchandani, 'A Si-Micromachined 48-Stage Knudsen pump for On-Chip Vacuum', J. Micromechanics Microengineering, vol. 22, no. 
10, 2012, doi: 10.1088/0960-1317/22/10/105026.

[61] M. Rojas Cardenas, I. Graur, P. Perrier, and J. G. Meolans, 'Thermal Transpiration Flow: A Circular Cross-Section Microtube Submitted to a Temperature Gradient', Phys. Fluids, vol. 23, no. 3, pp. 1-5, 2011, doi: 10.1063/1.3561744.

[62] C. K. Chen and H. C. Weng, 'Developing Natural Convection with Thermal Creep in a Vertical Microchannel', J. Phys. D. Appl. Phys., vol. 39, no. 14, pp. 3107-3118, 2006, doi: 10.1088/0022-3727/39/14/034.

[63] A. Mohammadzadeh, A. S. Rana, and H. Struchtrup, 'Thermal Stress vs. Thermal Transpiration: A Competition in Thermally Driven Cavity Flows', Phys. Fluids, vol. 27, no. 11, 2015, doi: 10.1063/1.4934624.

[64] K. Aoki, Y. Sone, and Y. Waniguchi, 'A Rarefied Gas Flow Induced by a Temperature Field: Numerical Analysis of The Flow between Two Coaxial Elliptic Cylinders with Different Uniform Temperatures', Comput. Math. with Appl., vol. 35, no. 1-2, pp. 15-28, 1998, doi: 10.1016/s0898-1221(97)00255-1.

[65] A. Mohammadzadeh and H. Struchtrup, 'Velocity Dependent Maxwell Boundary Conditions in DSMC', Int. J. Heat Mass Transf., vol. 87, pp. 151-160, 2015, doi: 10.1016/j.ijheatmasstransfer.2015.03.045.

[66] M. Kitamura, M. Tsutahara, S. Taguchi, R. Mitani, M. Enomura, and X. Zhang, 'A Study of Promotion of Sublimation Phenomenon of Freeze', pp. 1-7, 2017.

[67] S. Nakaye, H. Sugimoto, N. K. Gupta, and Y. B. Gianchandani, 'Thermal Method of Gas Separation with Micro-Pores', Proc. IEEE Sensors, vol. 2014-Decem, no. December, pp. 815-818, 2014, doi: 10.1109/ICSENS.2014.6985124.

[68] Y. Sone, 'Vacuum Pump without a Moving Part and its Performance', pp. 10411048, 2003, doi: 10.1063/1.1581654.

[69] Y. Sone and M. Yoshimoto, 'Demonstration of a Rarefied Gas Flow Induced Near The Edge of a Uniformly Heated Plate', Phys. Fluids, vol. 9, no. 11, pp. 3530-3534, 1997, doi: $10.1063 / 1.869461$. 
[70] William Crookes, 'On Attraction and Repulsion Resulting from Radiation', R. Soc., vol. 164, pp. 501-527, 1874, doi: https://doi.org/10.1098/rstl.1874.0015.

[71] William Crookes, 'On Attraction and Repulsion Resulting from Radiation.-Part II', R. Soc., vol. 23, no. 156-163, 1875, [Online]. Available: https://doi.org/10.1098/rspl.1874.0054.

[72] D. M. Bond, V. Wheatley, and M. Goldsworthy, 'Numerical Investigation into The Performance of Alternative Knudsen Pump Designs', Int. J. Heat Mass Transf., vol. 93, pp. 1038-1058, 2016, doi: 10.1016/j.ijheatmasstransfer.2015.10.069.

[73] V. Hemadri, V. V. Varade, A. Agrawal, and U. V. Bhandarkar, 'Investigation of rarefied gas flow in microchannels of non-uniform cross section', Phys. Fluids, vol. 28, no. 2, 2016, doi: 10.1063/1.4942183.

[74] S. Roy, R. Raju, H. F. Chuang, B. A. Cruden, and M. Meyyappan, 'Modeling Gas Flow Through Microchannels and Nanopores', J. Appl. Phys., vol. 93, no. 8, pp. 48704879, 2003, doi: 10.1063/1.1559936.

[75] A. Gavasane, A. Agrawal, A. M. Pradeep, and U. Bhandarkar, 'Simulation of a Temperature Drop for The Flow of Rarefied Gases in Microchannels', Numer. Heat Transf. Part A Appl., vol. 71, no. 10, pp. 1066-1079, 2017, doi: 10.1080/10407782.2017.1330091.

[76] S. G. Kandlikar and M. E. Steinke, 'Examples Of Microchannel Mass Transfer Process In Biological Systems', in Proceedings of ICMM2003 Microchannels and Minichannels First International Conference on April 24-25, 2003, Rochester, New York, US, 2016, pp. 1-11.

[77] M. For, F. In, D. A. T. Micro, P. Scroll, and D. For, 'Microscale Thermophysical Engineering Report: A Model For Flows in Channels, Pipes, and Ducts at Micro and Nano Scales', no. March 2013, pp. 37-41, 2010.

[78] A. Beskok, G. E. Karniadakis, and W. Trimmer, 'Rarefaction and Compressibility Effects in Gas Microflows', vol. 118, 1996, [Online]. Available: http://fluidsengineering.asmedigitalcollection.asme.org/. 
[79] A. Beskok and G. E. Karniadakis, 'Simulation of Heat and Momentum Transfer in Complex Microgeometries', J. Thermophys. Heat Transf., vol. 8, no. 4, pp. 647-655, 1994, doi: $10.2514 / 3.594$.

[80] J. C. Harley, 'Gas Flow in MicroChannels', J. Fluid Mech., vol. 28, pp. 257-274, 1995.

[81] Y. Shi, T. S. Zhao, and Z. Guo, 'Lattice Boltzmann Simulation of Dense Gas Flows in Microchannels', Phys. Rev. E - Stat. Nonlinear, Soft Matter Phys., vol. 76, no. 1, pp. 18, 2007, doi: 10.1103/PhysRevE.76.016707.

[82] S. Colin, 'Rarefaction and Compressibility Effects on Steady and Transient Gas Flows in Microchannels', Microfluid. Nanofluidics, vol. 1, no. 3, pp. 268-279, 2005, doi: 10.1007/s10404-004-0002-y.

[83] Z. Y. Guo and Z. X. Li, 'Size Effect on Single-Phase Channel Flow and Heat Transfer at Microscale', Int. J. Heat Fluid Flow, vol. 24, no. 3, pp. 284-298, 2003, doi: 10.1016/S0142-727X(03)00019-5.

[84] Y. Luo, G. Liu, L. L. Zou, B. K. Yu, and X. D. Wang, 'Thermal Behavior Investigation of Silicon-Pyrex Micro Heat Pipe', AIP Adv., vol. 4, no. 3, pp. 0-7, 2014, doi: 10.1063/1.4861209.

[85] H. Yamaguchi, M. Rojas-Cárdenas, P. Perrier, I. Graur, and T. Niimi, 'Thermal Transpiration Flow Through a Single Rectangular Channel', J. Fluid Mech., vol. 744, pp. 169-182, 2014, doi: 10.1017/jfm.2014.70.

[86] P. Yi, R. A. Awang, W. S. T. Rowe, K. Kalantar-Zadeh, and K. Khoshmanesh, 'PDMS Nanocomposites for Heat Transfer Enhancement in Microfluidic Platforms', Lab Chip, vol. 14, no. 17, pp. 3419-3426, 2014, doi: 10.1039/c4lc00615a.

[87] H. Zhou, G. Cai, and B. He, 'Effects of Wall Conditions on Flow Field in a Circular Microchannel', Appl. Therm. Eng., vol. 109, pp. 217-226, 2016, doi: 10.1016/j.applthermaleng.2016.08.042.

[88] C. Aubert, S. Colin, and R. Caen, 'Unsteady Gaseous Flows in Tapered 
Microchannels', Thechnical Proc. 1998 Int. Conf. Model. Simul. Microsystems, no. Chapter 11, pp. 486-491, 1998.

[89] V. Varade, V. S. Duryodhan, A. Agrawal, A. M. Pradeep, A. Ebrahimi, and E. Roohi, 'Low Mach Number Slip Flow Through Diverging Microchannel', Comput. Fluids, vol. 111, pp. 46-61, 2015, doi: 10.1016/j.compfluid.2014.12.024.

[90] Z. Y. Guo and X. B. Wu, 'Further Study on Compressibility Effects on The Gas Flow and Heat Transfer in a Microtube', Microscale Thermophys. Eng., vol. 2, no. 2, pp. 111-120, 1998, doi: 10.1080/108939598200024.

[91] Y. Asako, T. Pi, S. E. Turner, and M. Faghri, 'Effect of Compressibility on Gaseous Flows in Micro-Channels', Int. J. Heat Mass Transf., vol. 46, no. 16, pp. 3041-3050, 2003, doi: 10.1016/S0017-9310(03)00074-7.

[92] K. J. Heywood, 'Fluid Flows in The Environment: An Introduction', Phys. Educ., vol. 28, no. 1, pp. 43-47, 1993, doi: 10.1088/0031-9120/28/1/008.

[93] S. Schneiderbauer and A. Prokop, 'The Atmospheric Snow-Transport Model: SnowDrift 3D', vol. 57, no. 203, pp. 526-542, 2011.

[94] H. Babinsky, 'How do Wings Work?', Phys. Educ., vol. 38, no. 6, pp. 497-503, 2003, doi: 10.1088/0031-9120/38/6/001.

[95] S. Schneiderbauer and M. Krieger, 'What do the Navier- Stokes Equations mean ?', vol. 015020, doi: 10.1088/0143-0807/35/1/015020.

[96] John D Anderson, 'Computational Fluid Dynamics: Chapter 2: Governing Equations of Fluid Dynamics', Comput. Fluid Dyn., pp. 15-51, 2009, [Online]. Available: http://link.springer.com/10.1007/978-3-540-85056-4.

[97] C. R. Lilley and J. E. Sader, 'Velocity Profile in The Knudsen Layer According to The Boltzmann Equation', Proc. R. Soc. A Math. Phys. Eng. Sci., vol. 464, no. 2096, pp. 2015-2035, 2008, doi: 10.1098/rspa.2008.0071.

[98] J. J. Shu, J. Bin Melvin Teo, and W. Kong Chan, 'Fluid Velocity Slip and 
Temperature Jump at a Solid Surface', Appl. Mech. Rev., vol. 69, no. 2, 2017, doi: 10.1115/1.4036191.

[99] 'On Stresses in Rarefied Gases Arising from Inequalities of Temperature', Proc. R. Soc. London, $\quad 1878, \quad$ [Online]. Available: https://www.jstor.org/stable/113680?seq=1\#page_scan_tab_contents.

[100] A. Burgdorfer, 'The Influence of the Molecular Mean Free Path on the Performance of Hydrodynamic Gas Lubricated Bearings’, pp. 5-9, 2019.

[101] R. A. Millikan, 'Coefficients of Slip in Gases and the Law of Reflection of Molecules from the Surfaces of Solids and Liquids', Phys. Rev. 21, vol. 21, no. 3, 1923, doi: https://doi.org/10.1103/PhysRev.21.217.

[102] K. S. Van Dyke, 'The Coefficients of Viscosity and Of-Slip of Air and Carbondioxide by the Rotating Cylinder Method.', Phys. Rev., vol. 21, no. 23, 1923, doi: https://doi.org/10.1103/PhysRev.21.250.

[103] Leland Johnson Stacy, 'A Determination by the Constant Deflection Method of the Value of the Coefficient of Slip for Rough and for Smooth Surfaces in Air', Phys. Rev., vol. 19, no. 3, pp. 239-249, 1923.

[104] J. A. Bentz, R. V. Tompson, and S. K. Loyalka, 'The Spinning Rotor Gauge: Measurements of Viscosity, Velocity Slip Coefficients, and Tangential Momentum Accommodation Coefficients for N2 and CH4', Vacuum, vol. 48, no. 10, pp. 817-824, 1997, doi: 10.1016/s0042-207x(97)00031-6.

[105] A. I. Omelik, 'Measurement of Momentum Transport to Surfaces of Different form in a Hypersonic Free-Molecular Stream', Fluid Dyn, vol. 12, pp. 633-636, 1977, doi: https://doi.org/10.1007/BF01089691.

[106] E. B. Arkilic, K. S. Breuer, and M. A. Schmidt, 'Mass Flow and Tangential Momentum Accomodation in Silicon Micromachined Channels', J. Fluid Mech., vol. 437, pp. 29-43, 2001, doi: 10.1017/S0022112001004128.

[107] S. Colin, P. Lalonde, and R. Caen, 'Validation of a Second-Order Slip Flow Model 
in Rectangular Microchannels', Heat Transf. Eng., vol. 25, no. 3, pp. 23-30, 2004, doi: 10.1080/01457630490280047.

[108] L. S. Pan, G. R. Liu, and K. Y. Lam, 'Determination of Slip Coefficient for Rarefied Gas Flows Using Direct Simulation Monte Carlo', J. Micromechanics Microengineering, vol. 9, no. 1, pp. 89-96, 1999, doi: 10.1088/0960-1317/9/1/312.

[109] J. Jang and S. T. Wereley, 'Gaseous Slip Flow Analysis of a Micromachined Flow Sensor for Ultra Small Flow Applications', J. Micromechanics Microengineering, vol. 17, no. 2, pp. 229-237, 2007, doi: 10.1088/0960-1317/17/2/007.

[110] D. Blanchard and P. Ligrani, 'Slip and Accommodation Coefficients from Rarefaction and Roughness in Rotating Microscale Disk Flows', Phys. Fluids, vol. 19, no. 6, 2007, doi: 10.1063/1.2739416.

[111] A. Agrawal and S. V. Prabhu, 'Survey on Measurement of Tangential Momentum Accommodation Coefficient', J. Vac. Sci. Technol. A Vacuum, Surfaces, Film., vol. 26, no. 4, pp. 634-645, 2008, doi: 10.1116/1.2943641.

[112] N. T. P. Le, C. White, J. M. Reese, and R. S. Myong, 'Langmuir-Maxwell and Langmuir-Smoluchowski Boundary Conditions for Thermal Gas Flow Simulations in Hypersonic Aerodynamics', Int. J. Heat Mass Transf., vol. 55, no. 19-20, pp. 5032-5043, 2012, doi: 10.1016/j.ijheatmasstransfer.2012.04.050.

[113] S. K. Dadzie and J. G. Méolans, 'Temperature Jump and Slip Velocity Calculations From an Anisotropic Scattering Kernel', Phys. A Stat. Mech. its Appl., vol. 358, no. 2-4, pp. 328-346, 2005, doi: 10.1016/j.physa.2005.04.013.

[114] H. P. Kauehpour, M. Faghri, and Y. Asako, 'Effects of Compressibility and Rarefaction on Gaseous Flows in Microchannels', Numer. Heat Transf. Part A Appl., vol. 32, no. 7, pp. 677-696, 1997, doi: 10.1080/10407789708913912.

[115] P. Taheri and M. Bahrami, 'Thermal Transpiration Flow in Annular Microchannels', ASME 2012 10th Int. Conf. Nanochannels, Microchannels, Minichannels Collocated with ASME 2012 Heat Transf. Summer Conf. ASME 2012 Fluids Eng. Div. 
Sum, ICNMM 2012, no. January 2015, pp. 183-193, 2012, doi: 10.1115/ICNMM201273006.

[116] J. A. Trangenstein and J. A. Trangenstein, Introduction to Finite Element Methods. 2013.

[117] Dassault Systems, 'Accurate Design Simulations Improve Product Development', SolidWorks Product Design, vol. 1, no. 2. pp. 1-17, 2019.

[118] 'Product:MEMS Module', COMSOL. https://www.comsol.com/mems-module (accessed Mar. 07, 2021).

[119] M. Rojas-Cárdenas, I. Graur, P. Perrier, and J. G. Méolans, 'Time-dependent experimental Analysis of a Thermal Transpiration Rarefied Gas Flow', Phys. Fluids, vol. 25, no. 7, 2013, doi: 10.1063/1.4813805.

[120] S. Colin, 'Single-Phase Gas Flow in Microchannels', in Heat Transfer and Fluid Flow in Minichannels and Microchannels, 2013, pp. 11-102.

[121] A. Griesmer, 'Sweep Your Meshes with Ease', 2013. https://www.comsol.com/blogs/meshing-sweep-your-meshes-with-ease/ (accessed Mar. 07, 2021).

[122] W. Frei, 'Improving Your Meshing with Swept Meshes', 2015. https://www.comsol.com/blogs/improving-your-meshing-with-swept-meshes/.

[123] A. Agrawal and S. V. Prabhu, 'Survey on measurement of tangential momentum accommodation coefficient', J. Vac. Sci. Technol. A Vacuum, Surfaces, Film., vol. 26, no. 4, pp. 634-645, 2008, doi: 10.1116/1.2943641.

[124] V. Hemadri, A. Agrawal, and U. V. Bhandarkar, 'Determination of Tangential Momentum Accommodation Coefficient and Slip Coefficients for Rarefied Gas Flow in a Microchannel', Sadhana - Acad. Proc. Eng. Sci., vol. 43, no. 10, pp. 1-7, 2018, doi: 10.1007/s12046-018-0929-4.

[125] G. L. Morini, 'Laminar-to-Turbulent Flow Transition in Microchannels', 
Microscale Thermophys. Eng., vol. 8, no. 1, pp. 15-30, 2004, doi: 10.1080/10893950490272902.

[126] Y. P. Cheng, C. J. Teo, and B. C. Khoo, 'Microchannel Flows with Superhydrophobic surfaces: Effects of Reynolds Number and Pattern Width to Channel Height Ratio', Phys. Fluids, vol. 21, no. 12, pp. 1-12, 2009, doi: 10.1063/1.3281130.

[127] P. Kosky, R. Balmer, W. Keat, and G. Wise, 'Mechanical Engineering', in Exploring Engineering, Third., Elsevier Inc., Ed. Exploring Engineering, 2013, pp. 259281. 


\section{VITA AUCTORIS}

NAME:

PLACE OF BIRTH:

YEAR OF BIRTH:

EDUCATION:
Mukesh Arvind Raju

Mumbai, India

1993

Jeppiaar Engineering College, B.Eng., Old Mahabalipuram Road, Chennai-600119, Tamil Nadu, India. 2015

University of Windsor, M.A.Sc., Windsor, ON, 2021 